\title{
Analytic results for color-singlet production at NNLO QCD with the nested soft-collinear subtraction scheme
}

\author{
Fabrizio Caola ${ }^{1,2,3}$, Kirill Melnikov ${ }^{4}$, Raoul Röntsch ${ }^{4,5, a}$ \\ ${ }^{1}$ Rudolf Peierls Centre for Theoretical Physics, University of Oxford, Parks Road, Oxford OX1 3PU, UK \\ ${ }^{2}$ Wadham College, Oxford OX1 3PN, UK \\ 3 IPPP, Durham University, Durham DH1 3LE, UK \\ ${ }^{4}$ Institute for Theoretical Particle Physics, KIT, Karlsruhe, Germany \\ 5 Theoretical Physics Department, CERN, 1211, Geneva 23, Switzerland
}

Received: 15 February 2019 / Accepted: 13 April 2019 / Published online: 5 May 2019

(C) The Author(s) 2019

\begin{abstract}
We present analytic formulas that describe the fully-differential production of color-singlet final states in $q \bar{q}$ and $g g$ annihilation, including all the relevant partonic channels, through NNLO QCD. We work within the nested soft-collinear scheme, which allows the fully local subtraction of infrared divergences. We demonstrate analytic cancellation of soft and collinear poles and present formulas for the finite parts of all integrated subtraction terms. These results provide an important building block for calculating NNLO QCD corrections to arbitrary processes at hadron colliders within the nested soft-collinear subtraction scheme.
\end{abstract}

\section{Contents}

1 Introduction

2 Overview of the nested subtraction scheme . . . . 3

2.1 Extraction of soft singularities ....... . 4

2.2 Extraction of collinear singularities . . . . . . 5

3 Quark-initiated color-singlet production . . . . . . . 8

3.1 LO and NLO . . . . . . . . . . . . . . 8

3.2 NNLO: quark channels . . . . . . . . . . . 10

3.2.1 Terms with NNLO kinematics . . . . . 10

3.2.2 Tree-level terms with NLO kinematics . . 13

3.2.3 Tree-level terms with LO kinematics . . . 14

3.2.4 Terms involving virtual corrections . . . 15

3.3 NNLO: quark-gluon channels . . . . . . . 16

3.4 NNLO: gluon-gluon channel . . . . . . . . . 17

4 Gluon-initiated color-singlet production . . . . . . . 18

4.1 Spin correlations . . . . . . . . . . . 18

4.2 Definition of partonic channels . . . . . . 20

4.3 LO and NLO . . . . . . . . . . . 21

4.4 NNLO: gluon channel . . . . . . . . . . 21

a e-mail: raoul.rontsch@cern.ch
4.5 NNLO: quark-gluon channels . . . . . . . . 22

4.6 NNLO: $q \bar{q}$ channel . . . . . . . . . . . . . 23

4.7 NNLO: quark channels . . . . . . . . . . . . 24

5 Validation of results . . . . . . . . . . . 25

6 Conclusions ................. . 26

A Purely virtual contribution: definitions . . . . . . . . 27

A.1 Finite remainder: Drell-Yan . . . . . . . . 27

A.2 Finite remainder: Higgs . . . . . . . . . . 28

B Real-virtual contribution: definitions . . . . . . . . . 28

C Splitting functions . . . . . . . . . . . . . . . . 29

D Transition functions . . . . . . . . . . . . . . . 29

References . . . . . . . . . . . . . . 30

\section{Introduction}

Perturbative computations play an important role in the contemporary exploration of particle physics at the Large Hadron Collider (LHC). In particular, the lack of direct evidence for physics beyond the Standard Model suggests that further progress in particle physics will require a better understanding of hard hadron collisions and a confrontation of precise theoretical predictions with experimental results.

The quality of theoretical predictions for hard processes at the LHC has improved dramatically in recent years thanks to the advent of flexible methods for handling infrared singularities, which has led to the calculation of next-to-nextto-leading-order (NNLO) QCD corrections for sufficiently complex processes [1-20]. In spite of these successes, there are ongoing efforts to either simplify and improve existing methods or to devise "better" ones. For example, it is believed that the slicing formalism of Refs. [15-18] may be made more efficient through a deeper understanding of power corrections to soft and collinear limits [21-26]. Similarly, improved con- 
trol of the interplay between soft and collinear dynamics may lead to the formulation of simple "minimal" local subtraction schemes [27-29]. Although there is no guarantee that any of these efforts will result in an absolute breakthrough in fixed-order calculations for LHC processes, it is plausible that these developments will lead to more efficient computational frameworks and enable precise phenomenological descriptions of complex multiparticle final states.

In Ref. [30] we have attempted to simplify the residueimproved subtraction scheme proposed in Ref. [10,11]. This subtraction scheme is very attractive because it is fully local, completely general and perfectly modular, so that the subtractions for a generic process are built from a relatively small set of basic ingredients. Its main disadvantages include a lack of physical transparency and a certain redundancy, as well as the numerical integration of the subtraction terms that may inadvertently impact its efficiency.

We have argued in Ref. [30] that QCD color coherence removes an interplay between the angles and energies of soft and collinear particles in gauge-invariant QCD amplitudes, thus leading to a minimal number of subtraction terms that need to be considered. Perhaps more importantly, since soft and collinear singularities are not intertwined, it is possible to separate them cleanly, removing unnecessary redundancies of the subtraction procedure presented in Ref. [10,11]. As shown in Ref. [30], it appears to be advantageous to first subtract the double-soft singularities from the full amplitude and then to remove the remaining singularities iteratively. Once this is done, a transparent and physically appealing subtraction scheme is obtained. Moreover, this scheme appears to be very efficient, at least in the color-singlet production case that we have studied up to now [30,31].

Given the improved efficiency and inherent simplicity of the subtraction scheme developed in Ref. [30], it is natural to investigate whether one could obtain analytic results for the integrated counterterms. The successful completion of this task would lead to the formulation of the first subtraction scheme applicable at the LHC that is both fully local and under complete analytic control. Although it is hard to say to what extent these nice features are actually important in practice, we do hope that they will lead to a very efficient subtraction framework for completely generic processes.

It is easy to identify the major obstacles to obtaining fully analytic subtraction schemes. Indeed, any NNLO subtraction scheme involves three "double-unresolved" contributions whose integration is highly non-trivial. They are (1) the double-soft emission of two partons with energies $E_{f_{1}} \sim E_{f_{2}} \ll \sqrt{s}$, where $\sqrt{s}$ is the center-of-mass energy of the partonic process; (2) the emission of two partons collinear to one of the incoming legs and (3) the emission of two partons collinear to one of the hard final-state legs. We note that as far as the numerical implementation of NNLO QCD corrections to a generic process is concerned, contributions
(1) and (2) are the most problematic. Indeed, for any splitting process, the integrated contribution (3) is a number of the form $a_{f} / \epsilon+b_{f}$, so that it can be calculated numerically once and for all. On the contrary, the integrated contributions (1) and (2) are functions of the relative angles between hard partons and the momentum fraction carried into the hard process, respectively. ${ }^{1}$ Close to the end-points, these functions may develop integrable singularities, making their numerical evaluation tedious.

In Ref. [32], some of us presented analytic results for the integrated double-soft subtraction term. In this paper, we will argue that a minor modification of the subtraction procedure described in Ref. [30] greatly simplifies the analytic integration of the triple-collinear subtraction terms. In fact, such an integration of all relevant triple-collinear subtraction terms has recently been performed in Ref. [33]. Thanks to these results, it is now possible to present a subtraction framework for the production of color-singlet particles at hadron colliders that is both fully analytic and fully local.

Although the production of color-singlets at NNLO QCD has been studied many times, including the development of public computer codes, even the simplest versions of these processes, such as $p p \rightarrow Z$ and $p p \rightarrow H$, are quite useful to us because NNLO QCD corrections to these processes are known analytically $[34,35]$. This feature allows us to check all the non-trivial ingredients of our computational framework to a very high accuracy. We believe such a validation is important in view of the expected application of the subtraction method to more complex cases in the future. Of course, it is also interesting to explore the performance of our subtraction scheme by considering a well-known process, where many benchmarks exist already.

Nevertheless, it should be clear that the goal of this paper is to present analytic formulas relevant for the production of generic color-singlet final states at a hadron collider, written in a way that will allow us to move beyond color-singlet production. For this reason we decided to avoid using simplifications that are particular to the cases of Drell-Yan or Higgs production.

The rest of this paper is organized as follows. In Sect. 2 we summarize the main features of the nested soft-collinear subtraction scheme of Ref. [30] and explain how we modified it to allow for the analytic integration of the triple-collinear subtraction terms. In Sect. 3 we provide formulas for the $q \bar{q}$ initiated production of the color-singlet final state. In Sect. 4 we provide formulas for gluon annihilation into a colorsinglet final state. We discuss the validation of our results

\footnotetext{
1 Although the computation of NNLO QCD corrections to a generic process requires all three contributions, for sufficiently simple processes only a subset is needed. For example, for color-singlet production only contributions (1) and (2) are needed, while for color singlet decay only (1) and (3) are required.
} 
in Sect. 5 and conclude in Sect. 6. A large number of useful formulas are collected in the appendices, as well as in an ancillary file attached to this paper.

\section{Overview of the nested subtraction scheme}

In this section we briefly review the method for computing NNLO QCD corrections described in Ref. [30] and explain how to modify it to simplify the analytic integration of the triple-collinear subtraction terms. We consider the production of a color-singlet state $V$ in hadronic collisions. We write the (fiducial) cross section as

$$
\begin{aligned}
\sigma_{f}= & \sum_{\substack{a, b \in\left[-n_{f}, n_{f}\right]\\
}} \int \mathrm{d} x_{1} \mathrm{~d} x_{2} f_{a}\left(x_{1}, \mu_{F}\right) \\
& \times f_{b}\left(x_{2}, \mu_{F}\right) \mathrm{d} \hat{\sigma}_{f_{a} f_{b}}\left(x_{1}, x_{2}, \mu_{R}, \mu_{F} ; \mathcal{O}\right),
\end{aligned}
$$

where $n_{f}$ is the number of light flavors, $\mathrm{d} \hat{\sigma}_{f_{a} f_{b}}$ is the partonic cross section in the $f_{a} f_{b}$ channel, and we employ the following notation for parton distributions functions: $f_{0}=g$ and $f_{ \pm 1, \pm 2, \pm 3, \pm 4, \pm 5}=\{d / \bar{d}, u / \bar{u}, s / \bar{s}, c / \bar{c}, b / \bar{b}\}$. Finally, $\mathcal{O}$ is a suitable infrared-safe observable that defines the fiducial volume.

We consider the perturbative expansion of the partonic cross section and write

$$
\begin{aligned}
& \mathrm{d} \hat{\sigma}_{f_{a} f_{b}}\left(x_{1}, x_{2}, \mu_{R}, \mu_{F}, \mathcal{O}\right) \\
& \quad=\mathrm{d} \hat{\sigma}_{f_{a} f_{b}}^{\mathrm{LO}}+\mathrm{d} \hat{\sigma}_{f_{a} f_{b}}^{\mathrm{NLO}}+\mathrm{d} \hat{\sigma}_{f_{a} f_{b}}^{\mathrm{NNLO}}+\mathcal{O}\left(\alpha_{s}^{q+3}\right) .
\end{aligned}
$$

Here $q=0$ for quark-initiated processes and $q=2$ for gluon-initiated processes, and we have suppressed the arguments of the functions on the right-hand side. We focus on the NNLO QCD contribution $\mathrm{d} \hat{\sigma}_{f_{a} f_{b}}^{\mathrm{NNLO}}$. It can be written as

$$
\mathrm{d} \hat{\sigma}_{f_{a} f_{b}}^{\mathrm{NNLO}}=\mathrm{d} \hat{\sigma}_{f_{a} f_{b}}^{\mathrm{RR}}+\mathrm{d} \hat{\sigma}_{f_{a} f_{b}}^{\mathrm{RV}}+\mathrm{d} \hat{\sigma}_{f_{a} f_{b}}^{\mathrm{VV}}+\mathrm{d} \hat{\sigma}_{f_{a} f_{b}}^{\mathrm{ren}},
$$

where

$$
\begin{aligned}
\mathrm{d} \hat{\sigma}_{f_{a} f_{b}}^{\mathrm{RR}}= & \frac{\mathcal{N}}{2 s} \int \mathrm{d} \operatorname{Lips}(V)\left[d f_{4}\right]\left[d f_{5}\right](2 \pi)^{d} \\
& \times \delta_{d}\left(p_{1}+p_{2}-p_{V}-p_{4}-p_{5}\right) \\
& \times\left|\mathcal{M}^{\text {tree }}\right|^{2}\left(p_{1}, p_{2}, p_{V}, p_{4}, p_{5}\right) \mathcal{O}\left(p_{V}, p_{4}, p_{5}\right)
\end{aligned}
$$

$$
\begin{aligned}
\mathrm{d} \hat{\sigma}_{f_{a} f_{b}}^{\mathrm{RV}}= & \frac{\mathcal{N}}{2 s} \int \mathrm{dLips}(V)\left[d f_{4}\right](2 \pi)^{d} \delta_{d}\left(p_{1}+p_{2}-p_{V}-p_{4}\right) \\
& \times 2 \operatorname{Re}\left[\mathcal{M}^{\text {tree }} \mathcal{M}^{1-\text { loop }, *}\right]\left(p_{1}, p_{2}, p_{V}, p_{4}\right) \mathcal{O}\left(p_{V}, p_{4}\right) \\
\mathrm{d} \hat{\sigma}_{f_{a} f_{b}}^{\mathrm{VV}}= & \frac{\mathcal{N}}{2 s} \int \operatorname{dLips}(V)(2 \pi)^{d} \delta_{d}\left(p_{1}+p_{2}-p_{V}\right) \\
& \times\left[2 \operatorname{Re}\left[\mathcal{M}^{\text {tree }} \mathcal{M}^{2-\text { loop }, *}\right]\right. \\
& \left.+\left|\mathcal{M}^{1-\text { loop }}\right|^{2}\right]\left(p_{1}, p_{2}, p_{V}\right) \mathcal{O}\left(p_{V}\right)
\end{aligned}
$$

and $\mathrm{d} \hat{\sigma}^{\text {ren }}$ contains all contributions that originate from the renormalization of input parameters, such as the strong coupling constant $\alpha_{s}$ and the parton distribution functions (PDFs). In Eq. (2.4), $\mathcal{N}$ is a normalization factor that takes into account color and spin averages, $s$ is the partonic centerof-mass energy squared, $\operatorname{dLips}(V)$ is the phase space for the final state $V$, and

$\left[d f_{i}\right]=\frac{\mathrm{d}^{d-1} p_{i}}{(2 \pi)^{d-1} 2 E_{i}} \theta\left(E_{\max }-E_{i}\right)$.

Here $d$ is the dimensionality of space-time that we use as the regularization parameter, and $E_{\max }$ is an arbitrary ${ }^{2}$ energy scale that is introduced for future convenience.

Each term in Eq. (2.3) is individually divergent. These divergences can either appear explicitly as poles in $\epsilon=$ $(4-d) / 2$ or be hidden inside phase-space integrals. The goal of any subtraction scheme is to extract these divergences and to arrive at the following representation of the NNLO contribution to the cross section

$\mathrm{d} \hat{\sigma}_{f_{a} f_{b}}^{\mathrm{NNLO}}=\mathrm{d} \hat{\sigma}_{V+2, f_{a} f_{b}}^{\mathrm{NNLO}}+\mathrm{d} \hat{\sigma}_{V+1, f_{a} f_{b}}^{\mathrm{NNLO}}+\mathrm{d} \hat{\sigma}_{V, f_{a} f_{b}}^{\mathrm{NNLO}}$,

where $\mathrm{d} \hat{\sigma}_{V+i}^{\mathrm{NNLO}}$ are finite quantities that involve contributions with $V$ and up to $i$ partons in the final state. We will refer to $\mathrm{d} \hat{\sigma}_{V+i}^{\mathrm{NNO}}$, with $i=2,1,0$, as terms with NNLO, NLO and LO kinematics, respectively.

In Ref. [30], we proposed a framework to achieve the separation of the NNLO contributions to the cross section as in Eq. (2.6). It is based on three ideas:

- a multiparticle phase space can be decomposed into a sum of elements (partitions) such that for each partition only a well-definite subset of particles gives rise to collinear singularities upon integration over the phase space of the final state partons. An early discussion of this idea can be found in papers on NLO QCD subtractions [36,37]; in the context of NNLO QCD calculations, it was reincarnated in Ref. [10,11];

- for each of these partitions there exists a phase space parametrization that allows the extraction of both soft and collinear singularities in a fully factorized form $[10,11]$;

\footnotetext{
2 The only requirement on $E_{\max }$ is that it should be at least as large as the maximum energy allowed by the momentum-conserving $\delta$-functions in Eq. (2.4). For simplicity, throughout this paper we use $E_{\max }=\sqrt{s} / 2$, where $\sqrt{s}$ is the partonic center-of-mass energy.
} 
- thanks to gauge invariance and color coherence [38], soft and collinear singularities are not entangled in QCD amplitudes, in contrast to individual diagrams [30].

We argued in Ref. [30] that these three points allow us to follow the so-called FKS subtraction scheme [36,37], developed for NLO QCD computations, and to perform the required soft and collinear subtractions in a nested way. As a consequence, the computational framework becomes very transparent physically and quite efficient numerically.

We will illustrate the main ideas of Ref. [30] by considering the double-real emission corrections to the Drell-Yan process $q \bar{q} \rightarrow V$ as an example, focusing on the most complicated $q\left(p_{1}\right) \bar{q}\left(p_{2}\right) \rightarrow V+g\left(p_{4}\right) g\left(p_{5}\right)$ channel. All other partonic channels can be dealt with along the same lines although the details can be somewhat different. ${ }^{3}$ We write the corresponding cross section as

$$
\begin{aligned}
2 s \cdot \mathrm{d} \hat{\sigma}_{q \bar{q}}^{\mathrm{RR}} & =\int\left[d f_{4}\right]\left[d f_{5}\right] \theta\left(E_{4}-E_{5}\right) F_{\mathrm{LM}, q \bar{q}}(1,2,4,5) \\
& \equiv\left\langle F_{\mathrm{LM}, q \bar{q}}(1,2,4,5)\right\rangle,
\end{aligned}
$$

where

$$
\begin{aligned}
& F_{\mathrm{LM}, q \bar{q}}(1,2,4,5) \\
& =\mathcal{N} \int \mathrm{dLips}(V)(2 \pi)^{d} \delta_{d}\left(p_{1}+p_{2}-p_{V}-p_{4}-p_{5}\right) \\
& \quad \times\left|\mathcal{M}_{q \bar{q} \rightarrow V+g g}^{\text {tree }}\right|^{2}\left(p_{1}, p_{2}, p_{V}, p_{4}, p_{5}\right) \mathcal{O}\left(p_{V}, p_{4}, p_{5}\right),
\end{aligned}
$$

see Eq. (2.4). All energies in these formulas are to be understood in the center-of-mass frame of the colliding partons. Note that we have introduced the energy ordering $E_{4}>E_{5}$ in Eq. (2.7). Since the matrix element is symmetric with respect to the permutations of the gluons $g_{4}$ and $g_{5}$, we can remove the $1 / 2$ ! symmetry factor from $\mathcal{N}$.

Our goal is to extract singularities from Eq. (2.7). These singularities have different origins. There exist

- a double-soft singularity that occurs when the energies of the two gluons vanish in such a way that their ratio $E_{5} / E_{4}$ is fixed;

- a single-soft singularity that arises when $E_{5}$ vanishes at fixed $E_{4}$. Note that due to the energy ordering in Eq. (2.7) the opposite limit, $E_{4} \rightarrow 0$ at fixed $E_{5}$, cannot occur;

- many different collinear singularities that appear when one or both gluons are emitted along the direction of the incoming quark or the incoming anti-quark, or when the momenta of the two gluons become parallel to each other.

We need to extract all these singularities in an unambiguous way. We explain how to do this in the next two subsections.

\footnotetext{
3 Results for all the relevant channels are presented in the next sections.
}

\subsection{Extraction of soft singularities}

Before we begin extracting the singularities, we introduce the kinematic variables that we will use throughout the rest of this paper. We define the invariants

$$
\begin{aligned}
s_{i j} & =\left(p_{i}+p_{j}\right)^{2}=2 p_{i} \cdot p_{j}=2 E_{i} E_{j}\left(1-\cos \theta_{i j}\right) \\
& =2 E_{i} E_{j} \rho_{i j}=4 E_{i} E_{j} \eta_{i j},
\end{aligned}
$$

where $\rho_{i j}=2 \eta_{i j}=1-\cos \theta_{i j}$, and $\theta_{i j}$ is the relative angle between partons $i$ and $j$. We stress that energies and angles are defined in the center-of-mass frame. From this definition it is clear that $0 \leq \eta_{i j} \leq 1$. It is also clear that $s_{12}=s$, the partonic center-of-mass energy that we have already introduced.

As we explained in Ref. [30], it is convenient to begin by regularizing the double-soft singularity $E_{4} \sim E_{5} \sim \lambda \sqrt{s} \rightarrow$ 0 . We write

$$
\begin{aligned}
\left\langle F_{\mathrm{LM}, q \bar{q}}(1,2,4,5)\right\rangle= & \left\langle S S F_{\mathrm{LM}, q \bar{q}}(1,2,4,5)\right\rangle \\
& +\left\langle(I-S S) F_{\mathrm{LM}, q \bar{q}}(1,2,4,5)\right\rangle,
\end{aligned}
$$

where $S S$ is an operator that extracts the double-soft $\lambda \rightarrow 0$ singular limit from $F_{\mathrm{LM}, q \bar{q}}$. To make this statement precise, when the operator $S S$ acts on $F_{L M}$, it removes the fourmomenta of the gluons from both the energy-momentum conserving $\delta$-function and the observable, and extracts the leading singular behavior from the matrix element squared. The result is

$$
S S F_{\mathrm{LM}, q \bar{q}}(1,2,4,5)=g_{s, b}^{4} \operatorname{Eik}(1,2,4,5) F_{\mathrm{LM}, q \bar{q}}(1,2),
$$

where $g_{s, b}$ is the bare strong coupling and $\operatorname{Eik}(1,2,4,5)$ is the square of the eikonal factor derived e.g. in Ref. [39]. It is also given in Ref. [30] using notation that is identical to what we use in this paper. Also, $F_{\mathrm{LM}, q \bar{q}}(1,2)$ is defined analogously to Eq. (2.8); it reads

$$
\begin{aligned}
\left\langle F_{\mathrm{LM}, q \bar{q}}(1,2)\right\rangle= & \mathcal{N} \int \operatorname{dLips}(V)(2 \pi)^{d} \delta_{d}\left(p_{1}+p_{2}-p_{V}\right) \\
& \times\left|\mathcal{M}_{q \bar{q} \rightarrow V}^{\text {tree }}\right|^{2}\left(p_{1}, p_{2}, p_{V}\right) \mathcal{O}\left(p_{V}\right) .
\end{aligned}
$$

This tree-level matrix element squared integrated over the Born phase space obviously provides the leading-order result for the observable $\mathcal{O}$.

We deal with the two terms on the right-hand side of Eq. (2.10) in very different ways. In the first term, thanks to Eq. (2.11), the hard matrix element decouples and only the eikonal factor needs to be integrated over the two-gluon phase space. In our original paper [30] this integral was calculated numerically but, since then, an analytic computation 
of this integral has become available [32]. The result reads ${ }^{4}$

$$
\begin{aligned}
& \frac{\left\langle S S F_{\mathrm{LM}, q \bar{q}}(1,2,4,5)\right\rangle}{a_{s, b}^{2} e^{-2 \epsilon L}\left\langle F_{\mathrm{LM}, q \bar{q}}(1,2)\right\rangle} \\
& =C_{F}^{2} D_{S}^{C_{F}}+C_{F} C_{A} D_{S}^{C_{A}}+C_{F} T_{R} n_{f} D_{S}^{n_{f}},
\end{aligned}
$$

where we have defined

$a_{s, b}=\frac{g_{s, b}^{2}}{8 \pi^{2}} \frac{(4 \pi)^{\epsilon}}{\Gamma(1-\epsilon)}$,

and

$L=\log \left(\frac{s}{\mu^{2}}\right)$.

In Eq. (2.13), the abelian part is known in a closed form

$$
\begin{aligned}
D_{S}^{C_{F}} & =\frac{2}{\epsilon^{4}} \frac{\Gamma^{4}(1-\epsilon)}{\Gamma^{2}(1-2 \epsilon)} \\
& =\frac{2}{\epsilon^{4}}-\frac{2 \pi^{2}}{3 \epsilon^{2}}-\frac{8 \zeta_{3}}{\epsilon}-\frac{2 \pi^{4}}{45}+\mathcal{O}(\epsilon),
\end{aligned}
$$

and the other two contributions are computed as an expansion in $\epsilon$

$$
\begin{aligned}
D_{S}^{C_{A}}=\frac{1}{2 \epsilon^{4}} & +\frac{11}{12 \epsilon^{3}}+\frac{1}{\epsilon^{2}}\left(-\frac{16}{9}-\frac{\pi^{2}}{4}+\frac{11}{3} \ln 2\right) \\
& +\frac{1}{\epsilon}\left(\frac{217}{54}-\frac{11 \pi^{2}}{36}-\frac{137}{18} \ln 2\right. \\
& \left.-\frac{11}{3} \ln ^{2} 2-\frac{21}{4} \zeta_{3}\right)-\frac{649}{81}+\frac{125 \pi^{2}}{216} \\
& -\frac{11 \pi^{4}}{80}+\frac{434}{27} \ln 2-\frac{11}{6} \pi^{2} \ln 2 \\
& +\frac{137}{18} \ln ^{2} 2+\frac{22}{9} \ln ^{3} 2-\frac{275}{12} \zeta_{3}, \\
D_{S}^{n_{f}}=- & \frac{1}{3 \epsilon^{3}}+\frac{1}{\epsilon}\left(\frac{13}{18}-\frac{4}{3} \ln 2\right) \\
& +\frac{1}{\epsilon}\left(-\frac{125}{54}+\frac{\pi^{2}}{9}+\frac{35}{9} \ln 2+\frac{4}{3} \ln ^{2} 2\right) \\
& +\frac{601}{162}-\frac{23 \pi^{2}}{108}-\frac{223}{27} \ln 2+\frac{2 \pi^{2}}{3} \ln 2 \\
& -\frac{35}{9} \ln ^{2} 2-\frac{8}{9} \ln ^{3} 2+\frac{25}{3} \zeta_{3} .
\end{aligned}
$$

The equivalent results for gluon-initiated color singlet production can be obtained by simply replacing $C_{F} \rightarrow C_{A}$ in Eq. (2.13).

We now turn to the second term in Eq. (2.10) where the double-soft divergences are already regularized but both the $E_{5} \rightarrow 0$ divergence at fixed $E_{4}$ and collinear divergences are still present. To extract them, we repeat the above procedure

\footnotetext{
${ }^{4}$ We note that the result in Eq. (2.13) also includes the $n_{f}$-part which originates from the radiation of a $q \bar{q}$ pair in the final state. We include it here for completeness.
}

and subtract the $E_{5} \rightarrow 0$ singularities at fixed $E_{4}$. We call the corresponding operator $S_{5}$ and write

$$
\begin{aligned}
& \left\langle(I-S S) F_{\mathrm{LM}, q \bar{q}}(1,2,4,5)\right\rangle \\
& =\left\langle(I-S S)\left(I-S_{5}\right) F_{\mathrm{LM}, q \bar{q}}(1,2,4,5)\right\rangle \\
& \quad+\left\langle S_{5}(I-S S) F_{\mathrm{LM}, q \bar{q}}(1,2,4,5)\right\rangle .
\end{aligned}
$$

When the operator $S_{5}$ acts on $F_{\mathrm{LM}, q \bar{q}}(1,2,4,5)$, it removes the gluon $g_{5}$ from the phase space and the observable, and extracts the leading singularity

$$
\begin{aligned}
& S_{5} F_{\mathrm{LM}, q \bar{q}}(1,2,4,5)=\frac{g_{s, b}^{2}}{E_{5}^{2}} \\
& \quad \times\left[\left(2 C_{F}-C_{A}\right) \frac{\rho_{12}}{\rho_{15} \rho_{25}}+C_{A}\left(\frac{\rho_{14}}{\rho_{15} \rho_{45}}+\frac{\rho_{24}}{\rho_{25} \rho_{45}}\right)\right] \\
& \quad \times F_{\mathrm{LM}, q \bar{q}}(1,2,4) .
\end{aligned}
$$

We remind the reader that $\rho_{i j}$ is related to the angle $\theta_{i j}$ between partons $i$ and $j$ by $\rho_{i j}=1-\cos \theta_{i j}$. We have also introduced

$$
\begin{aligned}
& \left\langle F_{\mathrm{LM}, q \bar{q}}(1,2,4)\right\rangle \\
& =\mathcal{N} \int \operatorname{dLips}(V)\left[d f_{4}\right](2 \pi)^{d} \delta_{d}\left(p_{1}+p_{2}-p_{V}-p_{4}\right) \times \\
& \quad\left|\mathcal{M}_{q \bar{q} \rightarrow V+g}^{\text {tree }}\right|^{2}\left(p_{1}, p_{2}, p_{V}, p_{4}\right) \mathcal{O}\left(p_{V}, p_{4}\right),
\end{aligned}
$$

see Eq. (2.8). From here on, we will omit the subscript on $\mathcal{M}$ indicating the partonic process. It is clear that the second term in Eq. (2.18) has a simplified (i.e. independent of $g_{5}$ ) matrix element. The integration over the energy and angles of the gluon $g_{5}$ can therefore be performed, and the remaining infrared divergences in the matrix element for the process $q \bar{q} \rightarrow V+g_{4}$ can be dealt with in a way that is similar to what is usually done in next-to-leading-order computations. On the other hand, the first term in Eq. (2.18) is now free of soft divergences but still contains collinear singularities. We explain how to extract them in the next subsection.

\subsection{Extraction of collinear singularities}

In the previous subsection we extracted soft singularities from the double-real emission contribution by writing it as

$$
\left\langle F_{\mathrm{LM}, q \bar{q}}(1,2,4,5)\right\rangle \rightarrow\left\langle(I-S S)\left(I-S_{5}\right) F_{\mathrm{LM}, q \bar{q}}(1,2,4,5)\right\rangle
$$$$
+ \text { simpler terms with reduced kinematics. }
$$

The procedure continues with the extraction of collinear singularities. This requires an additional step, similar to the energy ordering in Eq. (2.7). Indeed, we need to split the phase space into regions such that in each region only a limited subset of momentum configurations can lead to collinear singularities.

Doing that involves the first two points on the itemized list that we presented after Eq. (2.6). The first point is the phase space partitioning; our goal is to split the phase space so that 
collinear singularities are localized in a clean and physical way. For example, we may want to focus on the collinear emissions off the incoming quark or the collinear emissions off the incoming anti-quark, or on the collinear emission of the gluon $g_{4}$ off the quark and the gluon $g_{5}$ off the anti-quark etc.

We can do that by introducing a partition of unity and using it to split the phase space. We write

$1=\omega^{41,51}+\omega^{42,52}+\omega^{41,52}+\omega^{42,51}$.

For the double-collinear partitions $\{4 i, 5 j\}, i \neq j$, the damping factor $\omega^{4 i, 5 j}$ is engineered in such a way that collinear singularities in $\omega^{4 i, 5 j} F_{\mathrm{LM}, q \bar{q}}(1,2,4,5)$ arise only if the momentum $p_{4}$ is parallel to $p_{i}$ and/or the momentum $p_{5}$ is parallel to $p_{j}$. Conversely, in the triple-collinear partitions $\{4 i, 5 i\}, i=1,2$, the damping factor $w^{4 i, 5 i}$ is designed in such a way that only the $p_{4}\left\|p_{i}, p_{5}\right\| p_{i}$ and $p_{4} \| p_{5}$ momentum configurations lead to a singularity. Apart from these conditions, there is significant freedom in choosing the partition functions; we will present a possible choice in the forthcoming sections. ${ }^{5}$

Contributions from the double-collinear partitions $\omega^{41,52}, \omega^{42,51}$ can be computed right away since the singular limits are easy to establish and no overlapping singularities are present. For example, in the case of $\omega^{41,52}$, it is sufficient to use the angle between the three-momenta $p_{4}$ and $p_{1}$ and the angle between the three-momenta $p_{5}$ and $p_{2}$ as independent variables to describe the collinear singularities in this partition.

The situation is more complex for the triple-collinear partitions, where overlapping singularities are present. The complexity stems from the fact that different hierarchies between $\rho_{4 i}, \rho_{5 i}$ and $\rho_{45}$ lead to inequivalent limits in this case. To identify these limits and extract them in a unique way, we further partition the phase space into four sectors. Taking as an example the $w^{41,51}$ partition, we introduce four sectors as follows

$$
\begin{aligned}
1= & \theta\left(\rho_{51}<\frac{\rho_{41}}{2}\right)+\theta\left(\frac{\rho_{41}}{2}<\rho_{51}<\rho_{41}\right) \\
& +\theta\left(\rho_{41}<\frac{\rho_{51}}{2}\right)+\theta\left(\frac{\rho_{51}}{2}<\rho_{41}<\rho_{51}\right) \\
= & \theta^{(a)}+\theta^{(b)}+\theta^{(c)}+\theta^{(d)} .
\end{aligned}
$$

The four sectors in the partition $w^{42,52}$ are constructed analogously. It is clear that Eq. (2.23) acts in such a way that in each of the four sectors only a small number of singular collinear limits occurs. We then expect that by choosing an appropriate

\footnotetext{
5 We note that if one is only interested in color-singlet production, partitions can easily be avoided. Nevertheless, we stress that here we use this class of processes to present results that can be used as building blocks for NNLO calculations for generic processes. In the general case, partitions are crucial for the formalism presented here.
}

parametrization for each of the four sectors, these singularities can be isolated and extracted. A convenient phase space parametrization for each of the four sectors can be found in Refs. [10,11].

In each of the four sectors shown in Eq. (2.23), the nested subtraction of these collinear limits can then be performed in a similar manner to that discussed in connection with the soft limits. We sketch how to do this by considering sector (a) of the $w^{41,51}$ partition. Because of the angular ordering in Eq. (2.23), a double-collinear singularity in this sector can only occur if $p_{5} \| p_{1}$. Similar to the soft case, we isolate it by writing

$$
\begin{aligned}
& \left\langle\theta^{(a)} w^{41,51}\left(I-S_{5}\right)(I-S S) F_{\mathrm{LM}, q \bar{q}}(1,2,4,5)\right\rangle \\
& =+\left\langle\theta^{(a)} C_{51} w^{41,51}\left(I-S_{5}\right)(I-S S) F_{\mathrm{LM}, q \bar{q}}(1,2,4,5)\right\rangle \\
& \quad+\left\langle\theta^{(a)}\left(I-C_{51}\right) w^{41,51}\left(I-S_{5}\right)(I-S S) F_{\mathrm{LM}, q \bar{q}}(1,2,4,5)\right\rangle,
\end{aligned}
$$

where $C_{51}$ is an operator that extracts the most singular contribution in the collinear $5 \| 1$ limit from the quantity on the left-hand side of Eq. (2.24) and enforces this collinear limit on the damping factor $w^{41,51}$, the reduced matrix element, the momentum-conserving $\delta$-function and the observable $\mathcal{O}$. This amounts to the replacements $\rho_{51} \rightarrow 0$ and $p_{1} \rightarrow p_{1}^{\prime}=p_{1}\left(E_{1}-E_{5}\right) / E_{1}$ in these quantities. The result reads

$$
\begin{aligned}
& C_{51} w^{41,51} F_{\mathrm{LM}, q \bar{q}}(1,2,4,5) \\
& =-\tilde{w}_{5 \| 1}^{41,51} \frac{g_{s, b}^{2}}{p_{1} \cdot p_{5}} P_{q q}\left(\frac{E_{1}}{E_{1}-E_{5}}\right) F_{\mathrm{LM}, q \bar{q}}\left(1^{\prime}, 2,4\right),
\end{aligned}
$$

where $P_{q q}$ is the splitting function

$$
P_{q q}(z)=C_{F}\left[\frac{1+z^{2}}{1-z}-\epsilon(1-z)\right] \text {, }
$$

and

$\tilde{w}_{k \| l}^{4 i, 5 j}=\lim _{\rho_{k l} \rightarrow 0} w^{4 i, 5 j}$.

The " 1 '" notation in $F_{\mathrm{LM}, q \bar{q}}$ refers to the $p_{1} \rightarrow p_{1}^{\prime}$ substitution that we just described. Compared to the soft limits, there is an additional subtlety. Indeed, in our construction the angular part of the phase space is non-trivial. To unambiguously define the $C_{51}$ operator, we must specify its action on the gluons' phase space $\left[d f_{4}\right]\left[d f_{5}\right]$. A convenient choice, adopted already in Ref. [30], is to let $C_{51}$ act on it, i.e. to take the $\rho_{51} \rightarrow 0$ limit of the measure $\left[d f_{4}\right]\left[d f_{5}\right]$.

The right hand side of Eq. (2.24) includes a term with reduced kinematics, which can be dealt with using methods similar to the ones used in NLO computations, and another term that only contains a triple-collinear singularity. The latter occurs whenever 4|| $5 \|$, without further hierarchy 
between $\rho_{51}, \rho_{41}$ and $\rho_{45}$. To regulate this last singularity, we introduce a triple-collinear operator $\mathbb{C}_{1}$ and write

$$
\begin{aligned}
\left\langle\theta^{(a)}\right. & \left.\left(I-C_{51}\right) w^{41,51}\left(I-S_{5}\right)(I-S S) F_{\mathrm{LM}, q \bar{q}}(1,2,4,5)\right\rangle \\
= & +\left\langle\theta^{(a)} \mathbb{C}_{1}\left(I-C_{51}\right)\left(I-S_{5}\right)(I-S S) F_{\mathrm{LM}, q \bar{q}}(1,2,4,5)\right\rangle \\
& +\left\langle\theta^{(a)}\left(I-\mathbb{C}_{1}\right)\left(I-C_{51}\right) w^{41,51}\left(I-S_{5}\right)(I-S S)\right. \\
& \left.\times F_{\mathrm{LM}, q \bar{q}}(1,2,4,5)\right\rangle,
\end{aligned}
$$

where we used $\mathbb{C}_{1} w^{41,51}=\lim _{\rho_{51} \rightarrow 0, \rho_{41} \rightarrow 0, \rho_{45} \rightarrow 0}=1$, which immediately follows from the definition of $w^{41,51}$. Similar to the single-collinear case, the operator $\mathbb{C}_{1}$ extracts the most singular behavior from the matrix element in the limit $\rho_{41} \sim \rho_{51} \sim \rho_{45} \rightarrow 0$ and sets $p_{1} \rightarrow p_{1}^{\prime}=p_{1}\left(E_{1}-\right.$ $\left.E_{4}-E_{5}\right) / E_{1}$ in the reduced matrix element, momentumconserving $\delta$-function and observable $\mathcal{O}$. We obtain

$$
\begin{aligned}
& \mathbb{C}_{1} F_{\mathrm{LM}, q \bar{q}}(1,2,4,5) \\
& =g_{s, b}^{4}\left(\frac{2}{s_{145}}\right)^{2} P_{g g q}\left(s_{45},-s_{14},-s_{15} ; z_{4}, z_{5}\right) F_{\mathrm{LM}, q \bar{q}}\left(1^{\prime}, 2\right),
\end{aligned}
$$

where $s_{145}=s_{45}-s_{14}-s_{15}$ and $P_{g g q}$ is a triple-collinear splitting function [39] that depends on the invariants $s_{i j}$ and the momentum fractions $z_{i}=E_{i} /\left(E_{4}+E_{5}-E_{1}\right)$.

Note that in the triple-collinear limit the only effect of the gluon emission on the reduced matrix element and the kinematics of the initial state is the boost $p_{1} \rightarrow p_{1}^{\prime}=\left(E_{1}-\right.$ $\left.E_{4}-E_{5}\right) / E_{1} p_{1}$. Defining $z=\left(E_{1}-E_{4}-E_{5}\right) / E_{1}=-1 / z_{1}$, we can schematically write Eq. (2.28) as

$$
\begin{aligned}
& \left\langle\theta^{(a)}\left(I-C_{51}\right) w^{41,51}\left(I-S_{5}\right)(I-S S) F_{\mathrm{LM}, q \bar{q}}(1,2,4,5)\right\rangle \\
& =\int \mathrm{d} z\langle P(z)\rangle\left\langle F_{\mathrm{LM}, q \bar{q}}(z, 2)\right\rangle \\
& \quad+\left\langle\theta^{(a)}\left(I-\mathbb{C}_{1}\right)\left(I-C_{51}\right) w^{41,51}\left(I-S_{5}\right)(I-S S)\right. \\
& \left.\quad \times F_{\mathrm{LM}, q \bar{q}}(1,2,4,5)\right\rangle,
\end{aligned}
$$

where $\langle P(z)\rangle$ is the integral of the (soft-regulated) splitting function over the phase space of the unresolved gluons, with the constraints $E_{4}+E_{5}=(1-z) E_{1}$ and $E_{4}<E_{5}$. We note that the second term in Eq. (2.30) is free of all singularities, and can be integrated in four dimensions using standard Monte-Carlo techniques.

Although this discussion is valid for any triple-collinear operator $\mathbb{C}_{1}$ that extracts the corresponding triple-collinear singularity from the matrix element squared, we must specify the action of $\mathbb{C}_{1}$ on $\left[d f_{4}\right]\left[d f_{5}\right]$ and on the $P_{g g q}$ function itself to unambiguously define the subtraction framework. In Ref. [30], we let $\mathbb{C}_{1}$ act on both $\left[d f_{4}\right]\left[d f_{5}\right]$ and on the splitting function, i.e. we evaluated all the $s_{i j}$ invariants in Eq. (2.29) and the angular factors in the $\left[d f_{4}\right]\left[d f_{5}\right]$ phase space in the triple-collinear limit. While this is a valid option, it is not the only one. In fact, this choice makes the analytic integration over the angles of the unresolved partons rather complicated, since it constrains the internal rotational symmetry of the unresolved phase space and does not allow for simple reparametrizations.

To overcome these issues, we now define the operator $\mathbb{C}_{1}$ in such a way that it does not act on either $\left[d f_{4}\right]\left[d f_{5}\right]$ or on $P_{g g q}$. Rather, $\mathbb{C}_{1}$ acts on the momentum-conserving $\delta$-function and on the observable, and extracts the leading triple-collinear singularity from the matrix element according to Eq. (2.29), but it leaves the angular factors in the $\left[d f_{4}\right]\left[d f_{5}\right]$ phase space and all the $s_{i j}$ invariants in Eq. (2.29) untouched. This modification of the subtraction scheme leads to a simpler integration of the triple-collinear splitting function over the unresolved phase space. Indeed, such a calculation has recently been performed for all relevant triplecollinear splitting functions in Ref. [33].

The results of Ref. [33], combined with the integrated double-soft subtraction terms presented earlier in Ref. [32], allow us to promote the fully local subtraction framework of Ref. [30] to a fully analytic scheme. This implies that we can now check the cancellation of all infrared poles analytically and achieve faster and more stable physical predictions by using analytic formulas for all the integrated subtraction terms.

We will present the analytic formulas required for the computation of NNLO QCD corrections to the production of color-singlet final states in the remaining parts of this paper. However, before we do that, a general comment is in order. Indeed, as should be clear from the discussion in this section, our framework is highly modular; we believe that this modularity ensures that its generalization beyond color-singlet production will proceed seamlessly. Indeed, the only differences between the color-singlet production and the general case with colored partons in the final state are:

1. compared to color-singlet production, a generic process has a more complicated color structure and requires double-soft integrals that are functions of relative angles of pairs of hard emitters, rather than pure numbers as in Eq. (2.17). The results relevant for this case have been presented in Ref. [32];

2. a generic process also involves triple-collinear final state splitting. While the analytic integration of the relevant splitting functions over the unresolved triple-collinear phase space has not been performed for all possible splittings, in Ref. [33] it was shown that techniques used to deal with initial state splittings can be successfully applied to final splittings as well.

It follows that the most general ingredients required for computing NNLO QCD corrections to generic partonic processes at the LHC can be obtained. From that perspective, 
the analytic formulas presented in this paper provide important building blocks for such a generic computation and give an excellent starting point for its generalization that will be addressed in the future. For now, we will proceed with presenting analytic formulas for all partonic channels that may contribute to the production of color-singlet final states at a hadron collider.

\section{Quark-initiated color-singlet production}

In this section, we consider the production of a color-singlet final state

$p p \rightarrow V+X$,

to NNLO QCD accuracy for reactions that are quark-initiated at leading order. We refer to these processes as "Drell-Yan processes", however, we emphasize that the results presented in this section are applicable to any color-singlet production process which is quark-initiated at LO. Typical examples include $p p \rightarrow Z, W^{+}, \gamma^{*}, Z Z, W^{+} W^{-}, W Z, W H, Z H$ and so on.

Starting from Eq. (2.1), we find it convenient to group the different partonic channels in three categories

$$
\begin{aligned}
\mathrm{d} \sigma_{f}^{\mathrm{DY}}= & \int \mathrm{d} x_{1} \mathrm{~d} x_{2} \sum_{\substack{a, b \in\left[-n_{f}, n_{f}\right] \\
a, b \neq 0}} f_{a}\left(x_{1}\right) f_{b}\left(x_{2}\right) \mathrm{d} \hat{\sigma}_{f_{a} f_{b}}^{\mathrm{DY}}\left(x_{1}, x_{2}\right) \\
& +\int \mathrm{d} x_{1} \mathrm{~d} x_{2} \sum_{\substack{a \in\left[-n_{f}, n_{f}\right] \\
a \neq 0}}\left[f_{a}\left(x_{1}\right) g\left(x_{2}\right) \mathrm{d} \hat{\sigma}_{f_{a} g}^{\mathrm{DY}}\left(x_{1}, x_{2}\right)\right. \\
& \left.+g\left(x_{1}\right) f_{a}\left(x_{2}\right) \mathrm{d} \hat{\sigma}_{g f_{a}}^{\mathrm{DY}}\left(x_{1}, x_{2}\right)\right] \\
& +\int \mathrm{d} x_{1} \mathrm{~d} x_{2} g\left(x_{1}\right) g\left(x_{2}\right) \mathrm{d} \hat{\sigma}_{g g}^{\mathrm{DY}}\left(x_{1}, x_{2}\right) .
\end{aligned}
$$

We omit the dependence of $\mathrm{d} \sigma_{f}^{\mathrm{DY}}$ on the renormalization and factorization scales $\mu_{R}, \mu_{F}$ and the observable $\mathcal{O}$ to shorten the notation. The first term in Eq. (3.2), d $\hat{\sigma}_{f_{a} f_{b}}^{\mathrm{DY}}$, receives contributions from quark channels and is present at LO. The terms on the second line, d $\hat{\sigma}_{f_{a} g}^{\mathrm{DY}}$ and $\mathrm{d} \hat{\sigma}_{g f_{a}}^{\mathrm{DY}}$, start contributing at NLO, and the last term $\mathrm{d} \hat{\sigma}_{g g}^{\mathrm{DY}}$ appears for the first time at NNLO. In what follows, we will consider the LO, NLO and NNLO contributions in turn. To simplify the notation, we will omit the superscript DY for the rest of this section.

For the NNLO contribution, we will consider the different channels defined in Eq. (3.2) separately. We also find it convenient to split each of these channels further, according to the highest final state multiplicity that they involve, (cf. Eq. (2.6))

$\mathrm{d} \hat{\sigma}_{f_{a} f_{b}}^{\mathrm{NNLO}}=\mathrm{d} \hat{\sigma}_{V+2, f_{a} f_{b}}^{\mathrm{NNLO}}+\mathrm{d} \hat{\sigma}_{V+1, f_{a} f_{b}}^{\mathrm{NNLO}}+\mathrm{d} \hat{\sigma}_{V, f_{a} f_{b}}^{\mathrm{NNLO}}$.

Finally, we separate each $\mathrm{d} \hat{\sigma}_{V+i, f_{a} f_{b}}^{\mathrm{NNLO}}$ in the above equation into terms involving only tree-level matrix elements and terms involving loop corrections, by writing ${ }^{6}$

$$
\begin{aligned}
& \mathrm{d} \hat{\sigma}_{V+2, f_{a} f_{b}}^{\mathrm{NNLO}}=\mathrm{d} \hat{\sigma}_{1245, f_{a} f_{b}}^{\mathrm{NNLO}}, \\
& \mathrm{d} \hat{\sigma}_{V+1, f_{a} f_{b}}^{\mathrm{NNLO}}=\mathrm{d} \hat{\sigma}_{124, f_{a} f_{b}}^{\mathrm{NNLO}}+\mathrm{d} \hat{\sigma}_{\mathrm{virt}}^{\mathrm{NNLO}, f_{a} f_{b},} \\
& \mathrm{~d} \hat{\sigma}_{V, f_{a} f_{b}}^{\mathrm{NNLO}}=\mathrm{d} \hat{\sigma}_{12, f_{a} f_{b}}^{\mathrm{NNLO}}+\mathrm{d} \hat{\sigma}_{\mathrm{virt}_{12}, f_{a} f_{b}}^{\mathrm{NNLO}} .
\end{aligned}
$$

The term $\mathrm{d} \hat{\sigma}_{1245, f_{a} f_{b}}^{\mathrm{NNLO}}$ receives contributions from processes with NNLO-like kinematics (i.e. with two additional resolved partons in the final state), and corresponds to the fully subtracted real-real contribution. The remaining terms arise from integrated subtraction terms, $\alpha_{s}$ and parton distribution function renormalizations, and real-virtual and purely virtual corrections. The terms $\mathrm{d} \hat{\sigma}_{124, f_{a} f_{b}}^{\mathrm{NNLO}}$ and $\mathrm{d} \hat{\sigma}_{12, f_{a} f_{b}}^{\mathrm{NNLO}}$ only involve

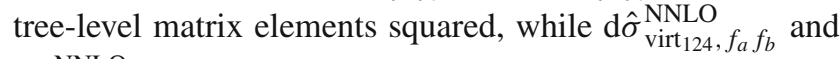
$\mathrm{d} \hat{\sigma}_{\text {virt }_{12}, f_{a} f_{b}}^{\mathrm{NNLO}}$ also involve finite remainders of virtual amplitudes. It is important to emphasize that all of the different terms in Eq. (3.4) are separately finite, so that we can discuss them separately. In what follows, we will present results for each of these terms.

\subsection{LO and NLO}

We start by discussing the quark channel $\mathrm{d} \hat{\sigma}_{f_{a} f_{b}}$, with $a, b \neq$ 0 , which is the only channel contributing at leading order. The LO cross section reads

$2 s \cdot \mathrm{d} \hat{\sigma}_{f_{a} f_{b}}^{\mathrm{LO}}=\left\langle F_{\mathrm{LM}, f_{a} f_{b}}(1,2)\right\rangle$.

We turn now to the NLO QCD corrections to the quark channel, following the discussion in Section 3 of Ref. [30] closely. The real emission corrections $q \bar{q} \rightarrow V+g$ have singularities associated with the radiated gluon becoming soft and/or collinear to the initial state quark or antiquark. We regulate these by writing

$$
\begin{aligned}
\langle & \left.F_{\mathrm{LM}, f_{a} f_{b}}(1,2,4)\right\rangle \\
= & \left\langle\hat{\mathcal{O}}_{\mathrm{NLO}} F_{\mathrm{LM}, f_{a} f_{b}}(1,2,4)\right\rangle+\left\langle S_{4} F_{\mathrm{LM}, f_{a} f_{b}}(1,2,4)\right\rangle \\
& +\left\langle\left(C_{41}+C_{42}\right)\left(I-S_{4}\right) F_{\mathrm{LM}, f_{a} f_{b}}(1,2,4)\right\rangle,
\end{aligned}
$$

where the soft operator $S_{4}$ and the collinear operators $C_{41}$ and $C_{42}$ behave as discussed in the previous section. The $\hat{\mathcal{O}}_{\mathrm{NLO}}$ operator reads

$\hat{\mathcal{O}}_{\mathrm{NLO}}=\left(I-C_{41}-C_{42}\right)\left(I-S_{4}\right)$,

\footnotetext{
${ }^{6}$ We note that certain partonic channels only contain a subset of these terms.
} 
and this makes the first term of Eq. (3.6) finite. The remaining terms in Eq. (3.6) are subtraction counterterms whose integration over the unresolved phase space will yield the singularities associated with the real emission. For color singlet production, the soft and soft-collinear limits cancel each other

$$
\left(I-C_{41}-C_{42}\right) S_{4} F_{\mathrm{LM}, f_{a} f_{b}}(1,2,4)=0 .
$$

Thus we only need to consider the pure collinear limits

$$
\left\langle\left(C_{41}+C_{42}\right) F_{\mathrm{LM}, f_{a} f_{b}}(1,2,4)\right\rangle \text {. }
$$

We focus on the $C_{41}$ operator, whose effect on $F_{\mathrm{LM}, f_{a} f_{b}}$ $(1,2,4)$ is

$$
\begin{aligned}
& C_{41} F_{\mathrm{LM}, f_{a} f_{b}}(1,2,4) \\
& =\frac{g_{s, b}^{2}}{E_{4}^{2} \rho_{41}}(1-z) P_{q q}(z) F_{\mathrm{LM}, f_{a} f_{b}}(z \cdot 1,2),
\end{aligned}
$$

where we have introduced the rescaling of the energies $z=1-E_{4} / E_{1}$ in this limit, and we recall

$$
P_{q q}(z)=C_{F}\left[\frac{1+z^{2}}{1-z}-\epsilon(1-z)\right] .
$$

Integrating over the angular phase space of $g_{4}$ and rewriting the integral over the energy $E_{4}$ as an integral over $z$ gives

$$
\begin{aligned}
& \left\langle C_{41} F_{\mathrm{LM}, f_{a} f_{b}}(1,2,4)\right\rangle \\
& =-\frac{a_{s}}{\epsilon} \frac{\Gamma^{2}(1-\epsilon)}{\Gamma(1-2 \epsilon)}\left(\frac{\mu^{2}}{s}\right)^{\epsilon} \\
& \quad \times \int_{0}^{1} \frac{\mathrm{d} z}{(1-z)^{2 \epsilon}} P_{q q}(z) \frac{F_{\mathrm{LM}, f_{a} f_{b}}(z \cdot 1,2)}{z},
\end{aligned}
$$

where $a_{s}=g_{s, b}^{2} /\left(8 \pi^{2}\right) \cdot(4 \pi)^{\epsilon} / \Gamma(1-\epsilon)$. The integration over $z$ still leads to divergences corresponding to the soft limit $z \rightarrow 1$. In order to make a connection with the collinear renormalization of the PDFs, we rewrite this in terms of plusdistributions [30] and perform the $\epsilon$-expansion. It is immediate to obtain

$$
\begin{aligned}
\left\langle C_{41} F_{\mathrm{LM}, f_{a} f_{b}}(1,2,4)\right\rangle & \\
= & -\frac{a_{s}}{\epsilon}\left(\frac{\mu^{2}}{s}\right)^{\epsilon} \frac{\Gamma^{2}(1-\epsilon)}{\Gamma(1-2 \epsilon)} \\
& \times\left[-\left(\frac{C_{F}}{\epsilon}+\frac{3 C_{F}}{2}\right)\left\langle F_{\mathrm{LM}, f_{a} f_{b}}(1,2)\right\rangle\right. \\
& \left.+\int_{0}^{1} \mathrm{~d} z \mathcal{P}_{q q}(z)\left\langle\frac{F_{\mathrm{LM}, f_{a} f_{b}}(z \cdot 1,2)}{z}\right\rangle\right],
\end{aligned}
$$

where we have defined $\mathcal{P}_{q q}(z)=\hat{P}_{q q}^{(0)}(z)+\epsilon \mathcal{P}_{q q}^{\prime}(z)+\mathcal{O}\left(\epsilon^{2}\right)$, with

$$
\hat{P}_{q q}^{(0)}(z)=C_{F}\left[\frac{2}{(1-z)_{+}}-(1+z)+\frac{3}{2} \delta(1-z)\right],
$$

and

$$
\begin{aligned}
\mathcal{P}_{q q}^{\prime}(z)= & -C_{F}[2(1+z) \ln (1-z)-(1-z) \\
& \left.-4\left[\frac{\ln (1-z)}{1-z}\right]_{+}\right] .
\end{aligned}
$$

In the above, $\hat{P}_{q q}^{(0)}(z)$ is the Altarelli-Parisi splitting function. We find it convenient to separate the $\delta$-function piece and write

$\hat{P}_{q q}^{(0)}(z)=\hat{P}_{q q, R}^{(0)}(z)+\hat{P}_{q q, \delta}^{(0)} \delta(1-z)$,

with $\hat{P}_{q q, \delta}^{(0)}=\gamma_{q}=3 C_{F} / 2$. A summary of the splitting functions required at both NLO and NNLO, for all the channels considered in this paper, is given in Appendix C.

After these manipulations, the cancellation of poles between the real emission correction, the virtual correction, and the PDF (and $\alpha_{s}$ ) renormalization can be performed immediately, and the $\epsilon \rightarrow 0$ limit can then be taken. The NLO QCD corrections in the quark channel then read

$$
\begin{aligned}
2 s \cdot \mathrm{d} \hat{\sigma}_{f_{a} f_{b}}^{\mathrm{NLO}}= & \left\langle F_{\mathrm{LV}, f_{a} f_{b}}^{\mathrm{fin}}(1,2)+\frac{\alpha_{s}(\mu)}{2 \pi}\left[\frac{2 \pi^{2}}{3} C_{F}\right.\right. \\
& \left.\left.-2 \gamma_{q} \ln \left(\frac{\mu^{2}}{s}\right)\right] F_{\mathrm{LM}, f_{a} f_{b}}(1,2)\right\rangle \\
& +\frac{\alpha_{s}(\mu)}{2 \pi} \int_{0}^{1} \mathrm{~d} z\left[\mathcal{P}_{q q}^{\prime}(z)-\hat{P}_{q q, R}^{(0)}(z) \ln \left(\frac{\mu^{2}}{s}\right)\right] \\
& \times\left\langle\frac{F_{\mathrm{LM}, f_{a} f_{b}}(z \cdot 1,2)+F_{\mathrm{LM}, f_{a} f_{b}}(1, z \cdot 2)}{z}\right\rangle \\
& +\left\langle\hat{\mathcal{O}}_{\mathrm{NLO}} F_{\mathrm{LM}, f_{a} f_{b}}(1,2,4)\right\rangle,
\end{aligned}
$$

with $a, b \neq 0$. In Eq. (3.17), $\left\langle F_{\mathrm{LV}, f_{a} f_{b}}^{\mathrm{fin}}(1,2)\right\rangle$ refers to the finite remainder of the (UV-renormalized) one-loop virtual correction. Its definition is given in Appendix A.

The $q g$ and $g q$ channels start contributing at NLO. They read

$$
\begin{aligned}
2 s \cdot \mathrm{d} \hat{\sigma}_{f_{a} g}^{\mathrm{NLO}}= & \frac{\alpha_{s}(\mu)}{2 \pi} \int_{0}^{1} \mathrm{~d} z \sum_{x}\left\langle\frac{F_{\mathrm{LM}, f_{a} f_{x}}(1, z \cdot 2)}{z}\right\rangle \\
& \times\left[\mathcal{P}_{q g}^{\prime}(z)-\hat{P}_{q g, R}^{(0)}(z) \ln \left(\frac{\mu^{2}}{s}\right)\right] \\
& +\left\langle\hat{\mathcal{O}}_{\mathrm{NLO}} F_{\mathrm{LM}, f_{a} g}(1,2,4)\right\rangle,
\end{aligned}
$$

and analogously 


$$
\begin{aligned}
2 s & \cdot \mathrm{d} \hat{\sigma}_{g f_{a}}^{\mathrm{NLO}}=\frac{\alpha_{s}(\mu)}{2 \pi} \\
& \times \int_{0}^{1} \mathrm{~d} z \sum_{x}\left[\mathcal{P}_{q g}^{\prime}(z)-\hat{P}_{q g, R}^{(0)}(z) \ln \left(\frac{\mu^{2}}{s}\right)\right] \\
& \times\left\langle\frac{F_{\mathrm{LM}, f_{x} f_{a}}(z \cdot 1,2)}{z}\right\rangle+\left\langle\hat{\mathcal{O}}_{\mathrm{NLO}} F_{\mathrm{LM}, g f_{a}}(1,2,4)\right\rangle,
\end{aligned}
$$

with $a \neq 0$. The splitting functions $\hat{P}_{q g, R}^{(0)}$ and $\mathcal{P}_{q g}^{\prime}$ are constructed along the same lines as $\hat{P}_{q q, R}^{(0)}$ and $\mathcal{P}_{q q}^{\prime}$ discussed above, and their definitions are given in Appendix C. Note that in these channels only a subset of soft/collinear singularities is present in $F_{\mathrm{LM}}(1,2,4)$; for example

$$
\begin{aligned}
& \hat{\mathcal{O}}_{\mathrm{NLO}} F_{\mathrm{LM}, q g}(1,2,4) \\
& \quad=\left(I-C_{41}-C_{42}\right)\left(I-S_{4}\right) F_{\mathrm{LM}, q g}(1,2,4) \\
& \quad=\left(I-C_{42}\right) F_{\mathrm{LM}, q g}(1,2,4) .
\end{aligned}
$$

\subsection{NNLO: quark channels}

In this section we consider the NNLO corrections to $\mathrm{d} \hat{\sigma}_{f_{a} f_{b}}$, with $a, b \neq 0$. This includes the partonic processes $q_{i} \bar{q}_{j} \rightarrow$ $V+g g, q_{i} \bar{q}_{j} \rightarrow V+q_{k} \bar{q}_{l}$ and $q_{i} q_{j} \rightarrow V+q_{k} q_{l}$. Of these partonic processes, the $q_{i} \bar{q}_{j} \rightarrow V+g g$ has the most complicated singularity structure; it was discussed in detail in Ref. [30] and reviewed in Sect. 2. Recall that we introduced an energy ordering $E_{4}>E_{5}$ (cf. Eq. (2.7)), which is natural since the amplitude is symmetric under the exchange of the two final state gluons.

The singularity structure is much simpler for final state quarks, where one could use only two sectors to separate the collinear singularities. Nevertheless, we find it convenient to treat the gluon and quark final states on an equal footing. We therefore need to symmetrize the amplitudes involving the final state quarks explicitly, since they are not symmetric in general; we do this by writing

$$
\begin{aligned}
\int & {\left[d f_{4}\right]\left[d f_{5}\right] F_{\mathrm{LM}, q \bar{q}}(1,2,4,5) } \\
= & \int\left[d f_{4}\right]\left[d f_{5}\right] F_{\mathrm{LM}, q \bar{q}}(1,2,4,5) \\
& \times\left[\theta\left(E_{4}>E_{5}\right)+\theta\left(E_{4}<E_{5}\right)\right] \\
= & \int\left[d f_{4}\right]\left[d f_{5}\right] \theta\left(E_{4}>E_{5}\right)\left[F_{\mathrm{LM}, q \bar{q}}(1,2,4,5)\right. \\
& \left.\left.+F_{\mathrm{LM}, q \bar{q}}(1,2,5,4)\right]\right] \\
\equiv & \left\langle F_{\mathrm{LM}}(1,2,4,5)\right\rangle .
\end{aligned}
$$

If one wishes to consider the final state gluons and quarks separately, one could do away with the energy ordering and the symmetrization of the quark amplitudes. We emphasize that in this case, the formulas in the forthcoming sections would require modifications.
As mentioned in Sect. 2, an important part of the subtraction scheme is the separation of the phase space into partitions such that in each partition, only a limited number of kinematic configurations leads to collinear divergences, $\mathrm{cf}$. Eq. (2.22). Throughout this paper, we choose the partition functions to be

$$
\begin{aligned}
w^{41,51} & =\eta_{42} \eta_{52}\left(1+\frac{\eta_{41}}{\eta_{45}+\eta_{42}+\eta_{51}}+\frac{\eta_{51}}{\eta_{45}+\eta_{41}+\eta_{52}}\right), \\
w^{42,52} & =\eta_{41} \eta_{51}\left(1+\frac{\eta_{42}}{\eta_{45}+\eta_{41}+\eta_{52}}+\frac{\eta_{52}}{\eta_{45}+\eta_{42}+\eta_{51}}\right), \\
w^{41,52} & =\frac{\eta_{42} \eta_{51} \eta_{45}}{\eta_{45}+\eta_{41}+\eta_{52}}, \quad w^{42,51}=\frac{\eta_{41} \eta_{52} \eta_{45}}{\eta_{45}+\eta_{42}+\eta_{51}},
\end{aligned}
$$

and recall that $\eta_{i j}=\rho_{i j} / 2$. It is straightforward to check that these functions restrict the collinear singularities as discussed in Sect. 2, and also that they sum up to one, cf. Eq. (2.22).

We now present results for the different terms in Eq. (3.4) that arise in the quark channel.

\subsubsection{Terms with NNLO kinematics}

This (hard) regularized contribution is the only one that involves the full matrix element for $f_{a} f_{b} \rightarrow V+f_{4} f_{5}$. It reads [30]

$$
\begin{aligned}
\mathrm{d} \hat{\sigma}_{1245, f_{a} f_{b}}^{\mathrm{NNLO}} & =\sum_{(i j) \in d c}\left\langle\left[\left(I-C_{5 j}\right)\left(I-C_{4 i}\right)\right][I-S S]\left[I-S_{5}\right]\right. \\
& \left.\times\left[d f_{4}\right]\left[d f_{5}\right] w^{4 i, 5 j} F_{\mathrm{LM}, f_{a} f_{b}}(1,2,4,5)\right\rangle \\
& +\sum_{i \in t c}\left\langle\left[\theta^{(a)}\left[I-\mathbb{C}_{i}\right]\left[I-C_{5 i}\right]+\theta^{(6)}\left[I-\boldsymbol{C}_{i}\right]\left[I-C_{45}\right]\right.\right. \\
& \left.+\theta^{(c)}\left[I-\boldsymbol{C}_{i}\right]\left[I-C_{4 i}\right]+\theta^{(d)}\left[I-\boldsymbol{C}_{i}\right]\left[I-C_{45}\right]\right] \\
& \left.\times[I-S S]\left[I-S_{5}\right]\left[d f_{4}\right]\left[d f_{5}\right] w^{4 i, 5 i} F_{\mathrm{LM}, f_{a} f_{b}}(1,2,4,5)\right\rangle .
\end{aligned}
$$

In this equation, $d c=\{(1,2),(2,1)\}$ and $t c=\{1,2\}$ refer to the double- and triple-collinear partitions, respectively, while the sectors $(a)-(d)$ are defined by the angular ordering conditions in Eq. (2.23). The operators $S_{5}, S S, C_{i j}$ and $\boldsymbol{C}_{i}$ have been discussed in great detail in Ref. [30], and in Sect. 2.

We note that $\mathrm{d} \hat{\sigma}_{1245 f_{a} f_{b}}^{\mathrm{NNLO}}$ is computed numerically in four dimensions. In order to do so, we must provide the explicit parametrization of the phase space for the complete final state which includes two radiated partons and a vector boson (or its decay products). It is clear that there are many different ways to do so. We find it useful to describe the phase space using tree-level variables, i.e. the invariant mass $M_{V}^{2}$ and the rapidity $Y$ of the vector boson, but other choices are 
possible. In addition, we have to choose the energies of the two final state partons and the relative angles between them and the hard emitter ${ }^{7}$ as independent variables, in order to extract singularities in the same way as in the computation of the integrated subtraction terms, which are presented in the forthcoming subsections. For this reason, there is less freedom in choosing how to parametrize the momenta of the radiated partons. We have discussed this point in some detail in Appendix B of Ref. [30]. We will not repeat this discussion, instead, our goal here is to provide a guide for a numerical implementation of Eq. (3.23).

We work in the center-of-mass frame of the colliding partons. As the first step, we determine the center-of-mass collision energy squared $s$. To do so, we parametrize the energies of the radiated partons as 8

$E_{4}=\frac{\sqrt{s}}{2} x_{1}, \quad E_{5}=\frac{\sqrt{s}}{2} x_{1} x_{2}$,

where $x_{1}, x_{2} \in[0: 1]$, and use momentum conservation $\left(p_{1}+p_{2}-p_{4}-p_{5}\right)^{2}=M_{V}^{2}$ to find

$s=\frac{M_{V}^{2}}{1-x_{1}\left(1+x_{2}\right)+x_{1}^{2} x_{2} \eta_{45}}$.

There is an obvious constraint $s<S_{h}$, where $S_{h}$ is the centerof-mass energy squared of the colliding hadrons, that we have to impose while generating the events.

The choice of angular variables depends on the partition and the sector; for the sake of definiteness, we will discuss the sector " $a$ " of the partition $w^{41,51}$. In this case, the scalar products $\eta_{i j}=\left(1-\cos \theta_{i j}\right) / 2$ may be parametrized by the variables $x_{3}, x_{4}, \lambda \in[0: 1]$ according to

$\eta_{41}=x_{3}, \quad \eta_{51}=x_{3} \frac{x_{4}}{2}, \quad \eta_{45}=\frac{x_{3}\left(1-x_{4} / 2\right)^{2}}{N_{F}\left(x_{3}, x_{4} / 2, \lambda\right)}$,

where

$$
\begin{aligned}
N_{F}\left(x_{3}, x_{4}, \lambda\right)= & 1+x_{4}\left(1-2 x_{3}\right)-2(1-2 \lambda) \\
& \sqrt{x_{4}\left(1-x_{3}\right)\left(1-x_{3} x_{4}\right)},
\end{aligned}
$$

see Refs. $[10,11]$. We note that, since $0 \leq x_{4} \leq 1$, the angular ordering $\eta_{51}<\eta_{41} / 2$ is assured.

In addition to the invariant mass, we also fix the rapidity of the vector boson in the laboratory frame, $Y$. This allows us to determine the fractions of the hadronic energy carried by the colliding partons, $\xi_{1,2}$. We find

$\xi_{1,2}=\sqrt{\frac{s}{S_{\mathrm{h}}}} e^{ \pm y}$,

where

$y=Y-\frac{1}{2} \ln \frac{1-x_{1}\left(1-\eta_{41}\right)-x_{1} x_{2}\left(1-\eta_{51}\right)}{1-x_{1}\left(1-\eta_{42}\right)-x_{1} x_{2}\left(1-\eta_{52}\right)}$.

7 The identity of the "hard emitter" depends on the partition.

${ }^{8}$ We remind the reader that we have chosen $E_{\max }=\sqrt{s} / 2$.
We require that $0<\xi_{1,2}<1$ and that both the numerator and the denominator in the argument of the logarithm in Eq. (3.29) are positive definite.

We are now in position to write down the four-momenta of the QCD partons for an event. We do so in the partonic center-of-mass frame. The knowledge of $\xi_{1,2}$ then allows us to boost momenta to the laboratory frame where all kinematic constraints are defined. The momenta read

$$
\begin{aligned}
p_{1,2} & =\frac{\sqrt{s}}{2}(1,0,0, \pm 1), \\
p_{4}= & \frac{\sqrt{s}}{2} x_{1}\left(1, \sin \theta_{41} \cos \varphi_{4}, \sin \theta_{41} \sin \varphi_{4}, \cos \theta_{41}\right), \\
p_{5}= & \frac{\sqrt{s}}{2} x_{1} x_{2}\left(1, \sin \theta_{51} \cos \left(\varphi_{4}+\varphi_{45}\right),\right. \\
& \left.\sin \theta_{51} \sin \left(\varphi_{4}+\varphi_{45}\right), \cos \theta_{51}\right),
\end{aligned}
$$

where $\cos \theta_{i j}=1-\rho_{i j}$ and $[10,11]$

$$
\begin{aligned}
& \sin \varphi_{45}=\frac{2 \sqrt{\lambda(1-\lambda)}\left(1-x_{4} / 2\right)}{N_{F}\left(x_{3}, x_{4} / 2, \lambda\right)}, \\
& \cos \varphi_{45}= \pm \sqrt{1-\sin ^{2} \varphi_{45}} .
\end{aligned}
$$

The four-momentum of the vector boson is obtained by momentum conservation $p_{V}=p_{1}+p_{2}-p_{4}-p_{5}$. If needed, further details of the colorless final state can be described. For example, in case of $V \rightarrow l^{+} l^{-}$, the phase space for leptonic decays is generated in the $V$-rest frame and the lepton momenta are boosted back into the partonic center-of-mass frame using the known $p_{V}$ and $M_{V}^{2}$.

For the chosen partition and sector, the phase space weight reads

$$
\begin{aligned}
& w_{h}\left(\left\{x_{i}\right\}, \lambda ;\left\{y_{i}\right\}\right) \\
& =\frac{w_{\mathrm{LO}}\left(\left\{y_{i}\right\}\right)}{\left(8 \pi^{2}\right)^{2}} \frac{s^{3}}{8 M_{V}^{2}} \frac{x_{1}^{3} x_{2} x_{3}\left(1-x_{4} / 2\right)}{N_{F}\left(x_{3}, x_{4} / 2, \lambda\right)} \\
& \quad \times w^{41,51}\left(\eta_{41}, \eta_{42}, \eta_{51}, \eta_{52}, \eta_{45}\right),
\end{aligned}
$$

where $w_{\mathrm{LO}}$ is the weight of the Born $f_{a} f_{b} \rightarrow V$ process, which depends in general on a set of variables $\left\{y_{i}\right\}$ that describes the $V$ final state. The contribution of the generated hard event to the phase-space integral is then

$$
\begin{aligned}
& I F_{\mathrm{LM}, q \bar{q}}(1,2,4,5) \\
& \quad \rightarrow \mathcal{N} f_{q}\left(\xi_{1}\right) f_{\bar{q}}\left(\xi_{2}\right) w_{h}\left(\left\{x_{i}\right\}, \lambda ;\left\{y_{i}\right\}\right)\left|\mathcal{M}\left(p_{1}, p_{2}, p_{V}, p_{4}, p_{5}\right)\right|^{2} .
\end{aligned}
$$

The matrix element squared can be calculated either in the center-of-mass frame or in the laboratory frame since all required boosts are defined at this point.

The contribution that we just described corresponds to the product of identity operators in Eq. (3.23); below we discuss how the subtraction terms in Eq. (3.23) are to be calculated. To this end, we consider first the class of terms in Eq. (3.23) 
where the double-soft operator $S S$ appears. We will start with the term $S S F_{\mathrm{LM}}$ and describe the weight of the counter-event produced by this term. To compute the weight, we take the limit $x_{1} \rightarrow 0$ everywhere; this corresponds to $E_{4,5} \rightarrow 0$ at $E_{5} / E_{4}$ held fixed. We obtain

$s_{\mathbb{S}}=M_{V}^{2}, \quad y_{\mathbb{S}}=Y, \quad \xi_{1,2}^{\mathbb{S}}=\sqrt{\frac{M_{V}^{2}}{S_{h}}} e^{ \pm Y}$.

The four-vectors for $p_{1,2}$ and $p_{4,5}$ are the same as in Eq. (3.30) but the four-momentum of the vector boson reads $p_{V}=p_{1}+p_{2}$, i.e. the radiation of the two soft partons does not impact the kinematics of the vector boson. The phase space weight of the counter-event reads

$$
\begin{aligned}
w_{\mathbb{S}} & \left(\left\{x_{i}\right\}, \lambda ;\left\{y_{i}\right\}\right) \\
= & \frac{w_{\mathrm{LO}}\left(\left\{y_{i}\right\}\right)}{\left(8 \pi^{2}\right)^{2}} \frac{M_{V}^{4}}{8} \frac{x_{1}^{3} x_{2} x_{3}\left(1-x_{4} / 2\right)}{N_{F}\left(x_{3}, x_{4} / 2, \lambda\right)} \\
& \times w^{41,51}\left(\eta_{41}, \eta_{42}, \eta_{51}, \eta_{52}, \eta_{45}\right),
\end{aligned}
$$

and its contribution to the fiducial cross section becomes

$$
\begin{aligned}
& S S F_{\mathrm{LM}, q \bar{q}}(1,2,3,4,5) \\
& \rightarrow \mathcal{N} f_{q}\left(\xi_{1}^{\mathbb{S}}\right) f_{\bar{q}}\left(\xi_{2}^{\mathbb{S}}\right) w_{\mathbb{S}}\left(\left\{x_{i}\right\}, \lambda ;\left\{y_{i}\right\}\right) \\
& \quad \operatorname{Eik}(1,2,4,5)\left|\mathcal{M}\left(p_{1}, p_{2}\right)\right|^{2} .
\end{aligned}
$$

Suppose we consider terms in Eq. (3.23) where, in addition to the double-soft operator $S S$, some other operator acts on $F_{\mathrm{LM}, q \bar{q}}$. In this case, we should just set the relevant variable(s) to zero in Eq. (3.36) and, if necessary, change the way the four-momenta are generated. For example, consider a term $C_{51} S S F_{\mathrm{LM}, q \bar{q}}(1,2,4,5)$. For this sector, the operator $C_{51}$ implies that $x_{4}$ should be set to zero everywhere after the leading $1 / x_{4}$ asymptotic limit is extracted. The fourmomenta are then unchanged, except for $p_{5}$ which becomes

$p_{5} \rightarrow C_{51} S S p_{5}=\frac{M_{V}}{2} x_{1} x_{2}(1,0,0,1)$.

Computing the $5 \| 1$ limit of the double-soft eikonal function, we arrive at the contribution from the $C_{51} S S F_{\mathrm{LM}, q \bar{q}}(1,2,4,5)$ kinematic configuration

$$
\begin{aligned}
& C_{51} S F_{\mathrm{LM}, q \bar{q}}(1,2,3,4,5) \\
& \left.\quad \rightarrow \mathcal{N} f_{q}\left(\xi_{1}^{\mathbb{S}}\right) f_{\bar{q}}\left(\xi_{2}^{\mathbb{S}}\right) w_{\mathbb{S}}\left(\left\{x_{i}\right\}, \lambda ;\left\{y_{i}\right\}\right)\right|_{x_{4} \rightarrow 0} \\
& \quad 16 C_{F}^{2} \frac{\tilde{s}_{12}}{\tilde{s}_{15} \tilde{s}_{25}} \frac{\tilde{s}_{12}}{\tilde{s}_{14} \tilde{s}_{24}}\left|\mathcal{M}\left(p_{1}, p_{2}\right)\right|^{2}
\end{aligned}
$$

The invariants $\tilde{s}_{i j}$ can be calculated by combining Eqs. (2.9) and (3.26), and by noting that in this limit the initial state partons are back-to-back, so $\eta_{12} \rightarrow 1$, and therefore $\eta_{42} \rightarrow$ $1-\eta_{41}$ and $\eta_{52} \rightarrow 1$. Explicitly, the $\tilde{s}_{i j}$ invariants read

$$
\begin{aligned}
& \tilde{s}_{12}=C_{51} S S s_{12}=4 E_{1} E_{2}, \\
& \tilde{s}_{14}=C_{51} S S s_{14}=4 E_{1} E_{4} x_{3}, \\
& \tilde{s}_{24}=C_{51} S s_{24}=4 E_{2} E_{4}\left(1-x_{3}\right), \\
& \tilde{s}_{15}=C_{51} S s_{15}=2 E_{1} E_{5} x_{3} x_{4}, \\
& \tilde{s}_{25}=C_{51} S S s_{25}=4 E_{2} E_{5} .
\end{aligned}
$$

Note that the invariant $\tilde{s}_{15} \neq 2\left(p_{1} \cdot p_{5}\right)$. This is so because the $1 / \tilde{s}_{15}$ term describes the leading $x_{4} \rightarrow 0$ singularity, so we must keep $x_{4} \neq 0$ there while setting $x_{4}$ to zero everywhere else.

As the last example, consider the triple-collinear limit, which corresponds to $x_{3} \rightarrow 0$, cf. Eqs. $(3.26,3.30)$. In this case, the partonic center-of-mass collision energy squared is

$s_{\boldsymbol{C}}=\frac{M_{V}^{2}}{1-x_{1}\left(1+x_{2}\right)}$.

Similar to the case of the hard event, we require $0<s_{\boldsymbol{C}}<S_{h}$. The four-momenta of partons to be used in the matrix element and the momentum-conserving $\delta$-function are

$$
\begin{aligned}
& p_{1,2}^{\boldsymbol{C}}=\mathbb{C} p_{1,2}=\frac{\sqrt{s_{\boldsymbol{C}}}}{2}(1,0,0, \pm 1), \\
& p_{4}^{\boldsymbol{C}}=\mathbb{C} p_{4}=\frac{\sqrt{s_{\boldsymbol{C}}}}{2} x_{1}(1,0,0,1), \\
& p_{5}^{\boldsymbol{C}}=\mathbb{C} p_{5}=\frac{\sqrt{s_{\boldsymbol{C}}}}{2} x_{1} x_{2}(1,0,0,1),
\end{aligned}
$$

and the vector boson four-momentum is

$$
\begin{aligned}
p_{V}^{\boldsymbol{C}} & =\left(p_{1}^{\boldsymbol{C}}-p_{4}^{\boldsymbol{C}}-p_{5}^{\boldsymbol{C}}\right)+p_{2}^{\boldsymbol{C}}=\frac{E_{1}-E_{4}-E_{5}}{E_{1}} p_{1}^{\boldsymbol{C}}+p_{2}^{\boldsymbol{C}} \\
& =\left(1-x_{1}\left(1+x_{2}\right)\right) p_{1}^{\boldsymbol{C}}+p_{2}^{\boldsymbol{C}} .
\end{aligned}
$$

Combining Eq. (3.42) with Eq. (3.40), we can easily check that $\left(p_{V}^{\boldsymbol{C}}\right)^{2}=M_{V}^{2}$ as, of course, it should.

As we emphasized in Sect. 2, we define $\mathbb{C}_{i}$ such that it does not act on the phase space. The weight of the counterevent is then identical to that of the hard process

$w_{\boldsymbol{C}}\left(\left\{x_{i}\right\}, \lambda ;\left\{y_{i}\right\}\right)=w_{h}\left(\left\{x_{i}\right\}, \lambda ;\left\{y_{i}\right\}\right)$

and the parameter $y$ relevant for calculating the fractions of the hadronic energy carried by the incoming partons reads

$y_{\boldsymbol{C}}=Y-\frac{1}{2} \ln \left(1-x_{1}\left(1+x_{2}\right)\right)$.

The momentum fractions themselves are then given by

$\xi_{1,2}^{\boldsymbol{C}}=\sqrt{\frac{\boldsymbol{s C}_{\boldsymbol{C}}}{S_{h}}} e^{ \pm y_{\boldsymbol{C}}}$.

Combining the different ingredients, we derive the weight of the triple-collinear counter-event

$$
\begin{aligned}
& \mathbb{C} F_{\mathrm{LM}, q \bar{q}}(1,2,4,5) \\
& \quad \rightarrow \mathcal{N} f_{q}\left(\xi_{1}^{C}\right) f_{\bar{q}}\left(\xi_{2}^{C}\right) w_{C}\left(\left\{x_{i}\right\}, \lambda ;\left\{y_{i}\right\}\right) \\
& \quad \times\left(\frac{2}{\bar{s}_{145}}\right)^{2} P_{g g q}\left(\bar{s}_{45},-\bar{s}_{14},-\bar{s}_{15} ; z_{4}, z_{5}\right)\left|\mathcal{M}\left(p_{1}^{\prime}, p_{2}\right)\right|^{2},
\end{aligned}
$$


where $p_{1}^{\prime}=\left(1-x_{1}\left(1+x_{2}\right)\right) p_{1}$. We evaluate the arguments of the triple-collinear splitting function $P_{g g q}$ without setting $x_{3}$ to zero. These invariants $\bar{s}_{i j}$ read

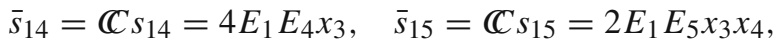

$\bar{s}_{45}=C s_{45}=\frac{4 E_{4} E_{5} x_{3}\left(1-x_{4} / 2\right)^{2}}{N_{F}\left(x_{3}, x_{4} / 2, \lambda\right)}$,

and $\bar{s}_{145}=\bar{s}_{45}-\bar{s}_{14}-\bar{s}_{15}$, while the energy fractions are $z_{i}=E_{i} /\left(E_{4}+E_{5}-E_{1}\right)$. We stress that the above scalar products in the splitting function are evaluated with $x_{3} \neq 0$, i.e. not in the triple-collinear limit.

The remaining contributions to the fully-subtracted cross section $\mathrm{d} \hat{\sigma}_{1245, f_{a} f_{b}}^{\mathrm{NNLO}}$ are computed along the same lines. The important thing is that we always take the leading singularity in the relevant variables and employ the limiting behavior of amplitudes-squared to calculate weights of the subtraction terms. We also make sure that the subtraction counterterms that make the hard matrix element finite are identical to the subtraction terms that have been analytically integrated.

\subsubsection{Tree-level terms with NLO kinematics}

In this section, we consider the term with NLO kinematics and tree-level matrix elements, $\mathrm{d} \hat{\sigma}_{124, f_{a} f_{b}}^{\mathrm{NNLO}}$. The general structure of this contribution is

$$
\begin{aligned}
\mathrm{d} \hat{\sigma}_{124, f_{a} f_{b}}^{\mathrm{NNLO}} & \frac{\alpha_{s}(\mu)}{2 \pi} \sum_{x} \int_{0}^{1} \mathrm{~d} z\left\{\hat{P}_{f_{x} f_{a}, R}^{(0)}(z)\right. \\
& \left\langle\ln \frac{\rho_{41}}{4} \hat{\mathcal{O}}_{\mathrm{NLO}}\left[\frac{\tilde{w}_{5 \| 1}^{41,51} F_{\mathrm{LM}, f_{x} f_{b}}(z \cdot 1,2,4)}{z}\right]\right\rangle \\
& +\left[\mathcal{P}_{f_{x} f_{a}}^{\prime}(z)-\hat{P}_{f_{x} f_{a}, R}^{(0)}(z) \ln \left(\frac{\mu^{2}}{s}\right)\right] \\
& \left\langle\hat{\mathcal{O}}_{\mathrm{NLO}}\left[\frac{F_{\mathrm{LM}, f_{x} f_{b}}(z \cdot 1,2,4)}{z}\right]\right\rangle+\left\langle\ln \frac{\rho_{42}}{4} \hat{\mathcal{O}}_{\mathrm{NLO}}\right. \\
& \left.\left.+\frac{\tilde{w}_{5 \| 2}^{42,52} F_{\mathrm{LM}, f_{a} f_{x}}(1, z \cdot 2,4)}{z}\right]\right\rangle \hat{P}_{f_{x} f_{b}, R}^{(0)}(z) \\
& +\left\langle\hat{\mathcal{O}}_{\mathrm{NLO}}\left[\frac{F_{\mathrm{LM}, f_{a} f_{x}}(1, z \cdot 2,4)}{z}\right]\right\rangle\left[\mathcal{P}_{f_{x} f_{b}}^{\prime}(z)\right. \\
& \left.\left.-\hat{P}_{f_{x} f_{b}, R}^{(0)}(z) \ln \left(\frac{\mu^{2}}{s}\right)\right]\right\} \\
& +\frac{\alpha_{s}(\mu)}{2 \pi}\left\langle\hat { \mathcal { O } } _ { \mathrm { NLO } } \left[\Delta_{q} \cdot F_{\mathrm{LM}, f_{a} f_{b}}(1,2,4)\right.\right. \\
& \left.\left.+r_{\mu} r_{v} F_{\mathrm{LM}, f_{a} f_{b}}^{\mu \nu}(1,2,4)\right]\right\rangle,
\end{aligned}
$$

where the various splitting functions are defined in Appendix C. Although we only need $\Delta_{q}$ in Eq. (3.48), it appears to be convenient to introduce a more general object $\Delta_{i \in\{q, g\}}$. It is defined as

$$
\begin{aligned}
\Delta_{i}= & C_{i}\left[\frac{2}{3} \pi^{2}-2 \ln \frac{2 E_{4}}{\sqrt{s}}\left(\tilde{w}_{5 \| 1}^{41,51} \ln \frac{\eta_{41}}{2}+\tilde{w}_{5 \| 2}^{42,52} \ln \frac{\eta_{42}}{2}\right)\right] \\
& +C_{A}\left[\ln \frac{2 E_{4}}{\sqrt{s}}\left(\ln \frac{2 E_{4}}{\sqrt{s}}+\ln \left(\eta_{41} \eta_{42}\right)\right)-\ln \eta_{41} \ln \eta_{42}\right] \\
& +\left(\frac{137}{18}-\frac{7}{6} \pi^{2}\right) C_{A}-\frac{13}{9} n_{f}+\beta_{0}\left[\tilde{w}_{4 \| \mid 5}^{41,51} \ln \frac{\eta_{42}}{\eta_{41}}\right. \\
& +\tilde{w}_{4|| 5}^{42,52} \ln \frac{\eta_{41}}{\eta_{42}}+\frac{\ln \left(\eta_{41} \eta_{42}\right)}{2} \\
& \left.-\ln \frac{2 E_{4}}{\sqrt{s}}+2 \ln 2\right]+\mathcal{X}_{i}\left[\ln \frac{2 E_{4}}{\sqrt{s}}+\frac{\ln \left(\eta_{41} \eta_{42}\right)}{2}\right] \\
& -2 \gamma_{i} \ln \left(\frac{\mu^{2}}{s}\right) .
\end{aligned}
$$

In the case $i=q$, we find $C_{q}=C_{F}, \gamma_{q}=3 C_{F} / 2$ and $\mathcal{X}_{q}=3 C_{A} / 2$, cf. Appendix. B. In addition

$\Delta^{r}=-\frac{C_{A}}{3}+\frac{n_{f}}{3}$.

Note that to obtain Eq. (3.49), we used $\eta_{41}+\eta_{42}=1$. In Eq. (3.48), $F_{\mathrm{LM}, f_{a} f_{b}}^{\mu \nu}$ is analogous to $F_{\mathrm{LM}, f_{a} f_{b}}$ but with the polarization vector for gluon 4 removed

$$
\begin{aligned}
\left\langle F_{\mathrm{LM}, f_{a} f_{b}}^{\mu \nu}(1,2,4)\right\rangle \\
=\mathcal{N} \int \mathrm{dLips}(V)\left[d f_{4}\right](2 \pi)^{d} \delta_{d}\left(p_{1}+p_{2}-p_{V}-p_{4}\right) \\
\quad \times\left[\mathcal{M}^{\text {tree }, \mu} \mathcal{M}^{*, \text { tree }, \nu}\right]\left(p_{1}, p_{2}, p_{V}, p_{4}\right) \mathcal{O}\left(p_{V}, p_{4}\right), \\
\mathcal{M}^{\text {tree }}=\epsilon_{\mu}\left(p_{4}\right) \mathcal{M}^{\text {tree }, \mu} .
\end{aligned}
$$

In Eq. (3.48), $F_{\mathrm{LM}}^{\mu \nu}$ is contracted with $r^{\mu} r^{\nu}$, where $r^{\mu}$ is a unit vector that spans the two-dimensional space orthogonal to $p_{4}$, see Ref. [30] for further details. If $p_{4}$ is parametrized as in Eq. (3.30), then

$r^{\mu}=\left(0,-\cos \theta_{41} \cos \varphi_{4},-\cos \theta_{41} \sin \varphi_{4}, \sin \theta_{41}\right)$.

Note that since $r \cdot p_{4}=0$ and $r^{2}=-1$, we can view $r^{\mu}$ as the polarization vector of the emitted gluon.

As an illustration, we now explicitly write Eq. (3.48) in the case of $Z$ production. For the same-flavor channel $\left(f_{a}, f_{b}\right)=$ $(q, \bar{q})$, Eq. (3.48) becomes

$$
\begin{aligned}
& \mathrm{d} \hat{\sigma}_{124, q \bar{q}}^{Z, \mathrm{NNLO}} \\
& =\frac{\alpha_{s}(\mu)}{2 \pi} \int_{0}^{1} \mathrm{~d} z\left\{\hat { P } _ { q q , R } ^ { ( 0 ) } ( z ) \left\langle\ln \frac{\rho_{41}}{4} \hat{\mathcal{O}}_{\mathrm{NLO}}\right.\right. \\
& \quad \times\left[\frac{\tilde{w}_{5 \| 1,1}^{41,51} F_{\mathrm{LM}, q \bar{q}}(z \cdot 1,2,4)}{z}\right]
\end{aligned}
$$




$$
\begin{aligned}
& \left.+\ln \frac{\rho_{42}}{4} \hat{\mathcal{O}}_{\mathrm{NLO}}\left[\frac{\tilde{w}_{5 \| \mid 2}^{42,52} F_{\mathrm{LM}, q \bar{q}}(1, z \cdot 2,4)}{z}\right]\right\rangle \\
& +\hat{P}_{g q, R}^{(0)}(z)\left\langle\ln \frac{\rho_{41}}{4} \hat{\mathcal{O}}_{\mathrm{NLO}} \times\left[\frac{\tilde{w}_{5 \| 1}^{41,51} F_{\mathrm{LM}, g \bar{q}}(z \cdot 1,2,4)}{z}\right]\right. \\
& \left.+\ln \frac{\rho_{42}}{4} \hat{\mathcal{O}}_{\mathrm{NLO}}\left[\frac{\tilde{w}_{5 \| \mid 2}^{42,52} F_{\mathrm{LM}, q g}(1, z \cdot 2,4)}{z}\right]\right\rangle \\
& +\left[\mathcal{P}_{q q}^{\prime}(z)-\hat{P}_{q q, R}^{(0)}(z) \ln \left(\frac{\mu^{2}}{s}\right)\right] \\
& \times\left\langle\hat{\mathcal{O}}_{\mathrm{NLO}}\left[\frac{F_{\mathrm{LM}, q \bar{q}}(z \cdot 1,2,4)+F_{\mathrm{LM}, q \bar{q}}(1, z \cdot 2,4)}{z}\right]\right\rangle \\
& +\left[\mathcal{P}_{g q}^{\prime}(z)-\hat{P}_{g q, R}^{(0)}(z) \ln \left(\frac{\mu^{2}}{s}\right)\right] \\
& \left.\times\left\langle\hat{\mathcal{O}}_{\mathrm{NLO}}\left[\frac{F_{\mathrm{LM}, g \bar{q}}(z \cdot 1,2,4)+F_{\mathrm{LM}, q g}(1, z \cdot 2,4)}{z}\right]\right\rangle\right\} \\
& +\frac{\alpha_{s}(\mu)}{2 \pi}\left\langle\hat { \mathcal { O } } _ { \mathrm { NLO } } \left[\Delta_{q} \cdot F_{\mathrm{LM}, q \bar{q}}(1,2,4)\right.\right. \\
& \left.\left.+\Delta^{r} \cdot\left[r_{\mu} r_{v} F_{\mathrm{LM}, q \bar{q}}^{\mu \nu}(1,2,4)\right]\right]\right\rangle .
\end{aligned}
$$

For different-flavor quark channels $\left(f_{a}, f_{b}\right)=\left(q_{i}, q_{j}\right)$ with $q_{i} \neq \bar{q}_{j}$, we find

$$
\begin{aligned}
\mathrm{d} \hat{\sigma}_{124, q_{i} q_{j}}^{Z, \mathrm{NNLO}}=\frac{\alpha_{s}(\mu)}{2 \pi} \int_{0}^{1} \mathrm{~d} z\left\{\hat { P } _ { g q , R } ^ { ( 0 ) } ( z ) \left\langle\ln \frac{\rho_{41}}{4} \hat{\mathcal{O}}_{\mathrm{NLO}}\right.\right. \\
\quad \times\left[\frac{\tilde{w}_{5 \| 1}^{41,51} F_{\mathrm{LM}, g q_{j}}(z \cdot 1,2,4)}{z}\right] \\
\left.\quad+\ln \frac{\rho_{42}}{4} \hat{\mathcal{O}}_{\mathrm{NLO}}\left[\frac{\tilde{w}_{5 \| \mid 2}^{42,52} F_{\mathrm{LM}, q_{i} g}(1, z \cdot 2,4)}{z}\right]\right\rangle \\
+\left[\mathcal{P}_{g q}^{\prime}(z)-\hat{P}_{g q, R}^{(0)}(z) \ln \left(\frac{\mu^{2}}{s}\right)\right] \\
\left.\quad \times\left\langle\hat{\mathcal{O}}_{\mathrm{NLO}}\left[\frac{F_{\mathrm{LM}, g q_{j}}(z \cdot 1,2,4)+F_{\mathrm{LM}, q_{i} g}(1, z \cdot 2,4)}{z}\right]\right\rangle\right\} .
\end{aligned}
$$

\subsubsection{Tree-level terms with LO kinematics}

We now turn to the contribution involving terms with LO kinematics and tree-level matrix elements, $\mathrm{d} \hat{\sigma}_{12, f_{a} f_{b}}^{\mathrm{NNO}}$. Accounting for the boost of the initial state along the collision axis, it can naturally be split into

$$
\begin{aligned}
\mathrm{d} \hat{\sigma}_{12, f_{a} f_{b}}^{\mathrm{NNLO}}= & \mathrm{d} \hat{\sigma}_{(z, \bar{z}), f_{a} f_{b}}^{\mathrm{NNO}}+\mathrm{d} \hat{\sigma}_{(z, 2), f_{a} f_{b}}^{\mathrm{NNLO}} \\
& +\mathrm{d} \hat{\sigma}_{(1, z), f_{a} f_{b}}^{\mathrm{NNL}}+\mathrm{d} \hat{\sigma}_{(1,2), f_{a} f_{b}}^{\mathrm{NNLO}},
\end{aligned}
$$

$a, b \neq 0$. We now consider each of these terms separately.
1. Terms involving $F_{\mathrm{LM}}(z \cdot 1, \bar{z} \cdot 2)$

$$
\begin{aligned}
& \mathrm{d} \hat{\sigma}_{(z, \bar{z}), f_{a} f_{b}}^{\mathrm{NNLO}}=\left(\frac{\alpha_{s}(\mu)}{2 \pi}\right)^{2} \sum_{x, y} \int_{0}^{1} \mathrm{~d} z \mathrm{~d} \bar{z} \\
& \times\left[\mathcal{P}_{f_{x} f_{a}}^{\prime}(z)-\ln \left(\frac{\mu^{2}}{s}\right) \hat{P}_{f_{x} f_{a}, R}^{(0)}(z)\right] \\
& \quad \times\left\langle\frac{F_{\mathrm{LM}, f_{x} f_{y}}(z \cdot 1, \bar{z} \cdot 2)}{z \bar{z}}\right\rangle \\
& \quad \times\left[\mathcal{P}_{f_{y} f_{b}}^{\prime}(\bar{z})-\ln \left(\frac{\mu^{2}}{s}\right) \hat{P}_{f_{y} f_{b}, R}^{(0)}(\bar{z})\right] .
\end{aligned}
$$

Once again, to illustrate this equation we consider the case of $Z$ production. Here, this contribution is only relevant for the $\left(f_{a}, f_{b}\right)=(q, \bar{q})$ channel, where it reads

$$
\begin{aligned}
\mathrm{d} \hat{\sigma}_{(z, \bar{z}), q \bar{q}}^{Z, \mathrm{NNLO}}=\left(\frac{\alpha_{s}(\mu)}{2 \pi}\right)^{2} \\
\quad \times \int_{0}^{1} \mathrm{~d} z \mathrm{~d} \bar{z}\left[\mathcal{P}_{q q}^{\prime}(z)-\ln \left(\frac{\mu^{2}}{s}\right) \hat{P}_{q q, R}^{(0)}(z)\right] \\
\quad \times\left\langle\frac{F_{\mathrm{LM}, q \bar{q}}(z \cdot 1, \bar{z} \cdot 2)}{z \bar{z}}\right\rangle \\
\quad \times\left[\mathcal{P}_{q q}^{\prime}(\bar{z})-\ln \left(\frac{\mu^{2}}{s}\right) \hat{P}_{q q, R}^{(0)}(\bar{z})\right] .
\end{aligned}
$$

2. Terms involving $F_{\mathrm{LM}}(z \cdot 1,2)$ and $F_{\mathrm{LM}}(1, z \cdot 2)$ :

$$
\begin{aligned}
\mathrm{d} \hat{\sigma}_{(z, 2), f_{a} f_{b}}^{\mathrm{NNLO}}+\mathrm{d} \hat{\sigma}_{(1, z), f_{a} f_{b}}^{\mathrm{NNLO}} & =\left(\frac{\alpha_{s}(\mu)}{2 \pi}\right)^{2} \sum_{x} \int_{0}^{1} \mathrm{~d} z\left[\mathcal{T}_{f_{x} f_{a}}(z)\left\langle\frac{F_{\mathrm{LM}, f_{x} f_{b}}(z \cdot 1,2)}{z}\right\rangle\right. \\
& \left.+\left\langle\frac{F_{\mathrm{LM}, f_{a} f_{x}}(1, z \cdot 2)}{z}\right\rangle \mathcal{T}_{f_{x} f_{b}}(z)\right] .
\end{aligned}
$$

This term has a non-trivial flavor structure. To simplify it, we employ the notation used to describe the NNLO QCD contributions to the Altarelli-Parisi splitting functions, and write the functions $\mathcal{T}$ in terms of nonsinglet, singlet, and vector functions

$$
\begin{aligned}
& \mathcal{T}_{q_{i} q_{j}}=\delta_{i j} \mathcal{T}_{q q}^{\mathrm{NS}}+\mathcal{T}_{q q}^{\mathrm{S}}, \\
& \mathcal{T}_{q_{i} \bar{q}_{j}}=\delta_{i j} \mathcal{T}_{q \bar{q}}^{\mathrm{V}}+\mathcal{T}_{q \bar{q}}^{\mathrm{S}} .
\end{aligned}
$$

Similar to the Altarelli-Parisi splitting functions, we have $\mathcal{T}_{q \bar{q}}^{\mathrm{S}}=\mathcal{T}_{q q}^{\mathrm{S}}$ through NNLO, and we will always use the latter in what follows. Again, we consider the example of $Z$ production. For the $\left(f_{a}, f_{b}\right)=(q \bar{q})$ channel, 
Eq. (3.58) becomes

$$
\begin{aligned}
\mathrm{d} \hat{\sigma}_{(z, 2), q \bar{q}}^{Z, \mathrm{NNLO}}+\mathrm{d} \hat{\sigma}_{(1, z), q \bar{q}}^{Z, \mathrm{NNLO}} \\
=\left(\frac{\alpha_{s}(\mu)}{2 \pi}\right)^{2} \int_{0}^{1} \mathrm{~d} z\left[\mathcal{T}_{q q}^{\mathrm{NS}}(z)+\mathcal{T}_{q q}^{\mathrm{S}}(z)\right] \\
\quad \times\left\langle\frac{F_{\mathrm{LM}, q \bar{q}}(z \cdot 1,2)+F_{\mathrm{LM}, q \bar{q}}(1, z \cdot 2)}{z}\right\rangle,
\end{aligned}
$$

while for the $q_{i} q_{j}$ with $i \neq-j$ it reads

$$
\begin{aligned}
\mathrm{d} \hat{\sigma}_{(z, 2), q_{i} q_{j}}^{Z, \mathrm{NNLO}}+\mathrm{d} \hat{\sigma}_{(1, z), q_{i} q_{j}}^{Z, \mathrm{NNLO}} \\
=\left(\frac{\alpha_{s}(\mu)}{2 \pi}\right)^{2} \int_{0}^{1} \mathrm{~d} z\left[\delta_{i j} \mathcal{T}_{q \bar{q}}^{\mathrm{V}}(z)+\mathcal{T}_{q q}^{\mathrm{S}}(z)\right] \\
\quad \times\left\langle\frac{F_{\mathrm{LM}, \bar{q}_{j} q_{j}}(z \cdot 1,2)+F_{\mathrm{LM}, q_{i}} \bar{q}_{i}(1, z \cdot 2)}{z}\right\rangle .
\end{aligned}
$$

The transition function $\mathcal{T}_{q q}^{\mathrm{NS}}$ is explicitly shown in Appendix D. All other $\mathcal{T}_{i j}$ functions are presented in an ancillary file.

3. Terms involving $F_{\mathrm{LM}}(1,2)$

$$
\begin{aligned}
\mathrm{d} \hat{\sigma}_{(1,2), f_{a} f_{b}}^{\mathrm{NNLO}} & \left\langle F_{\mathrm{LM}, f_{a} f_{b}}(1,2)\right\rangle \times\left(\frac{\alpha_{s}(\mu)}{2 \pi}\right)^{2} \\
& \left\{C _ { F } ^ { 2 } \left[\frac{8 \pi^{4}}{45}-\left(2 \pi^{2}+16 \zeta_{3}\right) \ln \left(\frac{\mu^{2}}{s}\right)\right.\right. \\
& \left.+\left(\frac{9}{2}-\frac{2 \pi^{2}}{3}\right) \ln ^{2}\left(\frac{\mu^{2}}{s}\right)\right] \\
& +C_{A} C_{F}\left[\frac{739}{81}+\frac{209 \pi^{2}}{72}-\frac{7 \pi^{4}}{80}+\ln 2\right. \\
& \times\left(\frac{4}{3}+\frac{11 \pi^{2}}{9}-\frac{7}{2} \zeta_{3}\right)+\left(\zeta_{2}-2\right) \ln ^{2} 2 \\
& -\frac{\ln ^{4} 2}{6}-\frac{407}{36} \zeta_{3}-4 \mathrm{Li}_{4}\left(\frac{1}{2}\right) \\
& +\ln \left(\frac{\mu^{2}}{s}\right)\left(-\frac{199}{54}+\frac{23 \pi^{2}}{24}-7 \zeta_{3}\right) \\
& \left.-\frac{11}{4} \ln ^{2}\left(\frac{\mu^{2}}{s}\right)\right]+C_{F} n_{f}\left[-\frac{214}{81}\right. \\
& -\frac{7 \pi^{2}}{18}-\ln 2\left(\frac{4}{3}+\frac{2 \pi^{2}}{9}\right)+2 \ln ^{2} 2 \\
& +\frac{37}{18} \zeta_{3}+\ln \left(\frac{\mu^{2}}{s}\right)\left(\frac{17}{27}-\frac{\pi^{2}}{12}\right)
\end{aligned}
$$

$$
\begin{aligned}
& \left.+\frac{1}{2} \ln ^{2}\left(\frac{\mu^{2}}{s}\right)\right]+\Theta_{b d}\left[\frac{23}{36} C_{F} n_{f}\right. \\
& \left.\left.+C_{A} C_{F}\left(\frac{\pi^{2}}{3}-\frac{131}{36}\right)+(2 \ln 2) C_{F} \beta_{0}\right]\right\} .
\end{aligned}
$$

The $\Theta_{b d}$ term in Eq. (3.62) depends on the choice of partition functions. It is defined as follows

$$
\begin{aligned}
\Theta_{b d} \equiv & -\left\langle[ I - C _ { 4 1 } - C _ { 4 2 } ] \left[\frac { \rho _ { 1 2 } } { \rho _ { 4 1 } \rho _ { 4 2 } } \left(\tilde{w}_{4 \| 5}^{41,51} \ln \frac{\eta_{41}}{1-\eta_{41}}\right.\right.\right. \\
& \left.\left.\left.+\tilde{w}_{4|| 5}^{42,52} \ln \frac{\eta_{42}}{1-\eta_{42}}\right)\right]\right\rangle .
\end{aligned}
$$

If the partition functions are chosen as in Eq. (3.22), it is immediate to obtain

$$
\Theta_{b d}=2-\frac{\pi^{2}}{3}
$$

\subsubsection{Terms involving virtual corrections}

Finally, we consider the two terms in Eq. (3.4) which involve virtual corrections, $\mathrm{d} \hat{\sigma}_{\text {virt } 124, f_{a} f_{b}}^{\mathrm{NNLO}}$ and $\mathrm{d} \hat{\sigma}_{\text {virt } 12, f_{a} f_{b}}^{\mathrm{NNLO}}$. The former corresponds to the real-virtual corrections, which have NLO kinematics. As such, they have singularities that appear when the radiated parton becomes unresolved. These singularities can be subtracted as at NLO, so that this term reads

$\mathrm{d} \hat{\sigma}_{\text {virt }_{124}, f_{a} f_{b}}^{\mathrm{NNLO}}=\left\langle\hat{\mathcal{O}}_{\mathrm{NLO}} F_{\mathrm{LV}, f_{a} f_{b}}^{\mathrm{fin}}(1,2,4)\right\rangle$,

where $F_{\mathrm{LV}, f_{a} f_{b}}^{\mathrm{fin}}(1,2,4)$ is a finite remainder of the one-loop amplitude, see Appendix B. The other term corresponds to virtual contributions with $\mathrm{LO}$ kinematics. It reads

$$
\begin{aligned}
\mathrm{d} \hat{\sigma}_{\mathrm{virt}}^{\mathrm{NNO}, f_{a} f_{b}} & \\
= & \left\langle F_{\mathrm{LVV}, f_{a} f_{b}}^{\mathrm{fin}}(1,2)+F_{\mathrm{LV}^{2}, f_{a} f_{b}}^{\mathrm{fin}}(1,2)\right. \\
& +\frac{\alpha_{s}(\mu)}{2 \pi}\left[\frac{2 \pi^{2}}{3} C_{F}-2 \gamma_{q} \ln \left(\frac{\mu^{2}}{s}\right)\right] \\
& \left.\times F_{\mathrm{LV}, f_{a} f_{b}}^{\mathrm{fin}}(1,2)\right\rangle+\frac{\alpha_{s}(\mu)}{2 \pi} \int_{0}^{1} d z \\
& \times\left[\mathcal{P}_{q q}^{\prime}(z)-\ln \left(\frac{\mu^{2}}{s}\right) \hat{P}_{q q, R}^{(0)}(z)\right] \\
& \times\left\langle\frac{F_{\mathrm{LV}, f_{a} f_{b}}^{\mathrm{fin}}(z \cdot 1,2)+F_{\mathrm{LV}, f_{a} f_{b}}^{\mathrm{fin}}(1, z \cdot 2)}{z}\right\rangle,
\end{aligned}
$$

where $\gamma_{q}=3 C_{F} / 2$ as before and $F_{\mathrm{LVV}}^{\mathrm{fin}}, F_{\mathrm{LV}^{2}}^{\mathrm{fin}}$ and $F_{\mathrm{LV}}^{\mathrm{fin}}$ are defined in Appendix A. 


\subsection{NNLO: quark-gluon channels}

In this section, we describe the NNLO contributions to the $q g$ channel, see Eq. (3.2). Similar results hold for the $g q$ channel. In principle, this channel could be treated in the same fashion as the quark channels discussed in the previous section. However, its singularity structure is much simpler, and so we need to consider a smaller number of limits. Indeed, no double-soft singularities are present in this case. Because of this, we find it convenient not to order the energies of partons 4 and 5. We write

$$
\int\left[d f_{4}\right]\left[d f_{5}\right] F_{\mathrm{LM}, q g}(1,2,4,5) \equiv\left\langle F_{\mathrm{LM}, q g}(1,2,4,5)\right\rangle,
$$

and parametrize $E_{4,5}=x_{1,2} E_{\max }$. However, the structure of the collinear singularities is similar to that discussed in Sect. 3.2, so we use the same angular parametrization and partitioning as defined there.

There is another important difference compared to the $q \bar{q}$ channel discussed in Sect. 3.2, namely that in the $q g$ channel spin correlations appear in the collinear emissions off the incoming gluon. We postpone their discussion to Sect. 4.1, where we consider the most general case of spin correlations. Apart from this, the structure of the result is very similar to the one discussed previously, so we limit ourselves to reporting the relevant formulas.

We write

$\mathrm{d} \hat{\sigma}_{f_{a} g}^{\mathrm{NNLO}}=\mathrm{d} \hat{\sigma}_{V+2, f_{a} g}^{\mathrm{NNLO}}+\mathrm{d} \hat{\sigma}_{V+1, f_{a} g}^{\mathrm{NNLO}}+\mathrm{d} \hat{\sigma}_{V, f_{a} g}^{\mathrm{NNLO}}$,

and

$\mathrm{d} \hat{\sigma}_{V+2, f_{a} g}^{\mathrm{NNLO}}=\mathrm{d} \hat{\sigma}_{1245, f_{a} g}^{\mathrm{NNLO}}$,

$\mathrm{d} \hat{\sigma}_{V+1, f_{a} g}^{\mathrm{NNLO}}=\mathrm{d} \hat{\sigma}_{124, f_{a} g}^{\mathrm{NNLO}}+\mathrm{d} \hat{\sigma}_{\mathrm{virt}_{124}, f_{a} g}^{\mathrm{NNLO}}$,

$\mathrm{d} \hat{\sigma}_{V, f_{a} g}^{\mathrm{NNLO}}=\mathrm{d} \hat{\sigma}_{(z, \bar{z}), f_{a} g}^{\mathrm{NNLO}}+\mathrm{d} \hat{\sigma}_{(1, z), f_{a} g}^{\mathrm{NNLO}}+\mathrm{d} \hat{\sigma}_{\operatorname{virt}_{12}, f_{a} g}^{\mathrm{NNLO}}$.

We consider the case with $a \neq 0$, and discuss each term separately. Note that in this channel there are no terms proportional to $F_{\mathrm{LM}}(1,2)$ or to $F_{\mathrm{LM}}(z \cdot 1,2)$ since the process $q g \rightarrow V$ at leading order is impossible. For the other terms, we obtain the following results.

\section{Tree-level terms with NNLO kinematics}

$$
\begin{aligned}
& \mathrm{d} \hat{\sigma}_{1245, f_{a} g}^{\mathrm{NNLO}}=\sum_{(i j) \in d c}\left\langle\left[\left(I-C_{5 j}\right)\left(I-C_{4 i}\right)\right]\left[I-S_{5}\right]\right. \\
& \left.\quad \times\left[d f_{4}\right]\left[d f_{5}\right] w^{4 i, 5 j} F_{\mathrm{LM}, f_{a} g}(1,2,4,5)\right\rangle \\
& \quad+\sum_{i \in t c} /\left[\theta^{(a)}\left[I-\mathbb{C}_{i}\right]\left[I-C_{5 i}\right]+\theta^{(b)}\left[I-\mathbb{C}_{i}\right]\left[I-C_{45}\right]\right.
\end{aligned}
$$

$$
\begin{aligned}
& \left.+\theta^{(c)}\left[I-\mathbb{C}_{i}\right]\left[I-C_{4 i}\right]+\theta^{(d)}\left[I-\mathbb{C}_{i}\right]\left[I-C_{45}\right]\right] \\
& \left.\times\left[I-S_{5}\right]\left[d f_{4}\right]\left[d f_{5}\right] w^{4 i, 5 i} F_{\mathrm{LM}, f_{a} g}(1,2,4,5)\right\rangle .
\end{aligned}
$$

The discussion of the individual terms is identical to that for the quark channels, cf. Sect. 3.2.1, with the only difference being that now the energies of the final state partons are parametrized differently.

\section{Tree-level terms with NLO kinematics}

$$
\begin{aligned}
\mathrm{d} \hat{\sigma}_{124, f_{a} g}^{\mathrm{NNO}}=\frac{\alpha_{s}(\mu)}{2 \pi} \int_{0}^{1} \mathrm{~d} z\left\{\hat { P } _ { q q , R } ^ { ( 0 ) } ( z ) \left\langle\ln \frac{\rho_{41}}{4} \hat{\mathcal{O}}_{\mathrm{NLO}}\right.\right. \\
{\left.\left[\frac{\tilde{w}_{5 \| 1}^{41,51} F_{\mathrm{LM}, f_{a} g}(z \cdot 1,2,4)}{z}\right]\right\rangle+\sum_{x \neq 0} \hat{P}_{q g, R}^{(0)}(z)\left\langle\ln \frac{\rho_{42}}{4} \hat{\mathcal{O}}_{\mathrm{NLO}}\right.} \\
\left.\quad \times\left[\frac{\left.\left.\tilde{w}_{5 \| \mid 2}^{42,52} F_{\mathrm{LM}, f_{a} f_{x}(1, z \cdot 2,4)}^{z}\right]\right\rangle+\hat{P}_{g g, R}^{(0)}(z)\left\langle\ln \frac{\rho_{42}}{4} \hat{\mathcal{O}}_{\mathrm{NLO}}\right.}{z}\right]\right\rangle \\
\left.\quad \times\left[\frac{\tilde{w}_{5 \| \mid 2}^{42,52} F_{\mathrm{LM}, f_{a} g}(1, z \cdot 2,4)}{z}\right]\right\rangle \\
+\left[\mathcal{P}_{q q}^{\prime}(z)-\hat{P}_{q q, R}^{(0)}(z) \ln \left(\frac{\mu^{2}}{s}\right)\right] \\
\times\left\langle\hat{\mathcal{O}}_{\mathrm{NLO}}\left[\frac{F_{\mathrm{LM}, f_{a} g}(z \cdot 1,2,4)}{z}\right]\right\rangle \\
+\sum_{x \neq 0}\left[\mathcal{P}_{q g}^{\prime}(z)-\hat{P}_{q g, R}^{(0)}(z) \ln \left(\frac{\mu^{2}}{s}\right)\right] \\
\times\left\langle\hat{\mathcal{O}}_{\mathrm{NLO}}\left[\frac{F_{\mathrm{LM}, f_{a} f_{x}}(1, z \cdot 2,4)}{z}\right]\right\rangle \\
+\left[\mathcal{P}_{g g}^{\prime}(z)-\hat{P}_{g g, R}^{(0)}(z) \ln \left(\frac{\mu^{2}}{s}\right)\right] \\
\times\left\langle\hat{\mathcal{O}}_{\mathrm{NLO}}\left[\frac{F_{\mathrm{LM}, f_{a} g}(1, z \cdot 2,4)}{z}\right]\right\rangle \\
+\frac{\alpha_{s}(\mu)}{2 \pi}\left\langle\hat{\mathcal{O}}_{\mathrm{NLO}}\left[\Delta^{(q g)} \cdot F_{\mathrm{LM}, f_{a} g}(1,2,4)\right]\right\rangle,
\end{aligned}
$$

which is analogous to Eq. (3.48) for the quark channels. The splitting functions in Eq. (3.71) are defined in Appendix $C$, and $\Delta^{(q g)}$ is given by

$$
\begin{aligned}
& \Delta^{(q g)}=C_{F}\left[\ln \frac{2 E_{4}}{\sqrt{s}}\left(\ln \frac{2 E_{4}}{\sqrt{s}}-2 \ln \eta_{41}-4 \ln 2\right)\right. \\
& +\left(\frac{3}{2}-2 \ln \frac{2 E_{4}}{\sqrt{s}}\right) \\
& \times\left(\tilde{w}_{4 \| 5}^{41,51} \ln \frac{\eta_{42}}{\eta_{41}}+\tilde{w}_{4 \|, 5}^{42,52} \ln \frac{\eta_{41}}{\eta_{42}}\right)+\frac{13}{2} \\
& +3 \ln 2-\pi^{2}+3 \ln \eta_{41} \\
& \left.+2 \operatorname{Li}_{2}\left(\eta_{42}\right)-\frac{3}{2} \ln \left(\frac{\mu^{2}}{s}\right)\right] \\
& +\beta_{0}\left[\frac{1}{2}\left(\ln \frac{2 E_{4}}{\sqrt{s}}+\ln \eta_{42}\right)-\ln \left(\frac{\mu^{2}}{s}\right)\right]
\end{aligned}
$$




$$
\begin{aligned}
& +C_{A}\left[\frac{\pi^{2}}{3}-\frac{3}{4} \ln \eta_{42}+\left(\frac{3}{2}-\ln \frac{2 E_{4}}{\sqrt{s}}\right)\right. \\
& \times \ln \frac{\eta_{42}}{\eta_{41}}-\frac{3}{4} \ln \frac{2 E_{4}}{\sqrt{s}} \\
& \left.-\operatorname{Li}_{2}\left(\eta_{42}\right)+\operatorname{Li}_{2}\left(\eta_{41}\right)\right] .
\end{aligned}
$$

3. Tree-level terms with LO kinematics involving $F_{\mathrm{LM}}(z$. $1, \bar{z} \cdot 2)$

$$
\begin{aligned}
\mathrm{d} \hat{\sigma}_{(z, \bar{z}), f_{a} g}^{\mathrm{NNLO}}= & \left(\frac{\alpha_{s}(\mu)}{2 \pi}\right)^{2} \sum_{x} \int_{0}^{1} \mathrm{~d} z \mathrm{~d} \bar{z} \\
& \times\left[\mathcal{P}_{q q}^{\prime}(z)-\ln \left(\frac{\mu^{2}}{s}\right) \hat{P}_{q q, R}^{(0)}(z)\right] \\
& \times\left\langle\frac{F_{\mathrm{LM}, f_{a} f_{x}}(z \cdot 1, \bar{z} \cdot 2)}{z \bar{z}}\right\rangle \\
& \times\left[\mathcal{P}_{q g}^{\prime}(\bar{z})-\ln \left(\frac{\mu^{2}}{s}\right) \hat{P}_{q g, R}^{(0)}(\bar{z})\right],
\end{aligned}
$$

which is analogous to Eq. (3.56).

4. Terms with $\mathbf{L O}$ kinematics involving $F_{\mathrm{LM}}(z \cdot 1,2)$ and $F_{\mathrm{LM}}(1, z \cdot 2)$

$$
\begin{aligned}
& \mathrm{d} \hat{\sigma}_{(1, z), f_{a} g}^{\mathrm{NNLO}} \\
& =\left(\frac{\alpha_{s}(\mu)}{2 \pi}\right)^{2} \sum_{x} \int_{0}^{1} \mathrm{~d} z \mathcal{T}_{q g}(z)\left\langle\frac{F_{\mathrm{LM}, f_{a} f_{x}}(1, z \cdot 2)}{z}\right\rangle,
\end{aligned}
$$

analogous to Eq. (3.58). The function $\mathcal{T}_{q g}$ can be found in the ancillary file.

5. Terms involving virtual corrections with NLO kinematics

$$
\mathrm{d} \hat{\sigma}_{\text {virt }_{124}, f_{a} g}^{\mathrm{NNLO}}=\left\langle\hat{\mathcal{O}}_{\mathrm{NLO}} F_{\mathrm{LV}, f_{a} g}^{\mathrm{fin}}(1,2,4)\right\rangle,
$$

analogous to Eq. (3.65). The finite remainder $F_{\mathrm{LV}, f_{a} g}^{\mathrm{fin}}$ $(1,2,4)$ is defined in Appendix B.

6. Terms involving virtual corrections with LO kinematics

$$
\begin{aligned}
\mathrm{d} \hat{\sigma}_{\text {virt }_{12}, f_{a} g}^{\mathrm{NNLO}}= & \frac{\alpha_{s}(\mu)}{2 \pi} \sum_{x} \int_{0}^{1}\left[\mathcal{P}_{q g}^{\prime}(z)-\ln \left(\frac{\mu^{2}}{s}\right) \hat{P}_{q g, R}^{(0)}(z)\right] \\
& \times\left\langle\frac{F_{\mathrm{LV}, f_{a} f_{x}}^{\mathrm{fin}}(1, z \cdot 2)}{z}\right\rangle
\end{aligned}
$$

analogous to Eq. (3.66). The finite remainder $F_{L V}^{\mathrm{fin}}$ is defined in Appendix A.
Results for the $g q$ channel can be obtained from the above formulas in a straightforward manner, by replacing labels $1 \leftrightarrow 2$.

\subsection{NNLO: gluon-gluon channel}

In this section, we describe the $g g$ channel, see Eq. (3.2). This case is particularly simple, since no soft or triple-collinear singularities are present. As the result, only double-collinear configurations need to be considered.

As a consequence, it is not necessary to partition the phase space in any way and the singularity structure can be dealt with as in NLO computations described earlier. We parametrize the energies and angles of the emitted partons as

$E_{4,5}=x_{1,2} E_{\max }, \quad \eta_{41}=x_{3}, \quad \eta_{51}=x_{4}$.

Singularities only appear when $x_{3,4}$ is equal to either zero or one. Because of the simplicity of the singularity structure, we treat the two cases at once. To deal with them, we use a similar but simpler strategy to the one discussed in the preceding sections. We write

$$
\begin{aligned}
& F_{\mathrm{LM}, g g}(1,2,4,5) \\
&= {\left[\left(I-C_{41}-C_{42}\right)+C_{41}+C_{42}\right]\left[\left(I-C_{51}-C_{52}\right)\right.} \\
&\left.+C_{51}+C_{52}\right] F_{\mathrm{LM}, g g}(1,2,4,5) \\
&= {\left[I-C_{41}-C_{42}-C_{51}-C_{52}+C_{42} C_{51}\right.} \\
&\left.+C_{41} C_{52}\right] F_{\mathrm{LM}, g g}(1,2,4,5) \\
&+\left[C_{41}+C_{42}+C_{51}+C_{52}-C_{42} C_{51}\right. \\
&\left.-C_{41} C_{52}\right] F_{\mathrm{LM}, g g}(1,2,4,5),
\end{aligned}
$$

where we used the fact that $C_{41} C_{51}=C_{42} C_{52} \rightarrow 0$ when it acts on the matrix element. The first term on the right hand side in Eq. (3.78) is free of singularities and corresponds to what we called $\mathrm{d} \hat{\sigma}_{1245}^{\mathrm{NNLO}}$ in the previous sections, while the second term contains the subtraction counterterms. We combine Eq. (3.78) with contributions from the PDF renormalization and write

$\mathrm{d} \hat{\sigma}_{g g}^{\mathrm{NNLO}}=\mathrm{d} \hat{\sigma}_{V+2, g g}^{\mathrm{NNLO}}+\mathrm{d} \hat{\sigma}_{V+1, g g}^{\mathrm{NNLO}}+\mathrm{d} \hat{\sigma}_{V, g g}^{\mathrm{NNLO}}$,

where

$\mathrm{d} \hat{\sigma}_{V+2, g g}^{\mathrm{NNLO}}=\mathrm{d} \hat{\sigma}_{1245, g g}^{\mathrm{NNLO}}$,
$\mathrm{d} \hat{\sigma}_{V+1, g g}^{\mathrm{NNLO}}=\mathrm{d} \hat{\sigma}_{124, g g}^{\mathrm{NNLO}}$,
$\mathrm{d} \hat{\sigma}_{V, g g}^{\mathrm{NNLO}}=\mathrm{d} \hat{\sigma}_{(z, \bar{z}), g g}^{\mathrm{NNLO}}$.

At variance to the cases discussed above, virtual corrections do not contribute in this channel. The individual terms read as follows. 


\section{Tree-level terms with NNLO kinematics}

$$
\begin{aligned}
& \mathrm{d} \hat{\sigma}_{1245, g g}^{\mathrm{NNLO}}=\left\langle\left[ I-C_{41}-C_{42}-C_{51}-C_{52}\right.\right. \\
& \left.\quad+C_{42} C_{51}+C_{41} C_{52}\right] \\
& \left.\quad \times\left[d f_{4}\right]\left[d f_{5}\right] F_{\mathrm{LM}, g g}(1,2,4,5)\right\rangle,
\end{aligned}
$$

where again the construction of each term follows the discussion in Sect. 3.2.1, but with the parametrization shown in Eq. (3.77).

\section{Tree-level terms with NLO kinematics}

$$
\begin{aligned}
& \mathrm{d} \hat{\sigma}_{124, g g}^{\mathrm{NNLO}} \\
& =\frac{\alpha_{s}(\mu)}{2 \pi} \sum_{x \neq 0} \int_{0}^{1} \mathrm{~d} z\left[\mathcal{P}_{q g}^{\prime}(z)-\hat{P}_{q g, R}^{(0)}(z) \ln \left(\frac{\mu^{2}}{s}\right)\right] \\
& \quad \times\left\langle\hat{\mathcal{O}}_{\mathrm{NLO}}\left[\frac{F_{\mathrm{LM}, f_{x} g}(z \cdot 1,2,4)+F_{\mathrm{LM}, g f_{x}}(1, z \cdot 2,4)}{z}\right]\right\rangle .
\end{aligned}
$$

3. Tree-level terms with LO kinematics involving $F_{\mathrm{LM}}(z$.

$$
1, \bar{z} \cdot 2)
$$

$$
\begin{aligned}
\mathrm{d} \hat{\sigma}_{(z, \bar{z}), g g}^{\mathrm{NNLO}}= & \left(\frac{\alpha_{s}(\mu)}{2 \pi}\right)^{2} \sum_{x, y} \int_{0}^{1} \mathrm{~d} z \mathrm{~d} \bar{z} \\
& \times\left[\mathcal{P}_{q g}^{\prime}(z)-\ln \left(\frac{\mu^{2}}{s}\right) \hat{P}_{q g}^{(0)}(z)\right] \\
& \times\left\langle\frac{F_{\mathrm{LM}, f_{x} f_{y}}(z \cdot 1, \bar{z} \cdot 2)}{z \bar{z}}\right\rangle \\
& \times\left[\mathcal{P}_{q g}^{\prime}(\bar{z})-\ln \left(\frac{\mu^{2}}{s}\right) \hat{P}_{q g}^{(0)}(\bar{z})\right] .
\end{aligned}
$$

\section{Gluon-initiated color-singlet production}

In this section, we consider the production of a color-singlet final state $H$ in gluon fusion at NNLO QCD. We refer to this process as "Higgs production", although, similar to the "Drell-Yan process" discussed in the previous section, these results are applicable to the production of any color-singlet final state which proceeds through gluon fusion at LO. The procedure of extracting the infrared divergences is identical to what has already been discussed in the case of the $q \bar{q}$ annihilation and we do not repeat it. However, in gluon-initiated processes, initial state radiation leads to spin correlations that we have not discussed up to now. In the next section we show how to deal with this complication.

\subsection{Spin correlations}

We have discussed spin correlations relevant to the computation of NNLO QCD corrections to the $q \bar{q} \rightarrow V$ process in Ref. [30]. In that case, the spin correlations appeared because of the splitting of a virtual gluon into two final state partons, $g^{*} \rightarrow f_{4,5}$. For the $g g \rightarrow H$ process, the situation is different in that spin correlations also appear in the initial state radiation, including its triple-collinear limit. In this section, we discuss this case.

We will begin with the discussion of NLO QCD corrections to $g g \rightarrow H$. The computation proceeds exactly as for the $q \bar{q} \rightarrow V$ process, cf. Sect. 3.1. However, when the collinear operator acts on the matrix element squared, we find

$$
\begin{aligned}
& \left\langle C_{41} F_{\mathrm{LM}, g g}(1,2,4)\right\rangle \\
& =-\left\langle\frac{g_{s, b}^{2}}{p_{1} \cdot p_{4}} P_{g g, \mu \nu}\left(z^{-1}\right) F_{\mathrm{LM}, g g}^{\mu \nu}(z \cdot 1,2)\right\rangle,
\end{aligned}
$$

where the splitting function reads

$$
\begin{aligned}
& P_{g g}^{\mu \nu}(z) \\
& =2 C_{A}\left[\left(\frac{1-z}{z}+\frac{z}{1-z}\right)\left[-g_{\perp, d-2}^{\mu \nu}\right]\right. \\
& \left.\quad-2(1-\epsilon) z(1-z) \frac{k_{\perp}^{\mu} k_{\perp}^{\nu}}{k_{\perp}^{2}}\right] .
\end{aligned}
$$

The transverse momentum $k_{\perp}$ is defined using the Sudakov decomposition

$p_{4}=(1-z) p_{1}+\beta p_{2}+k_{\perp}$,

where $k_{\perp} \cdot p_{1}=k_{\perp} \cdot p_{2}=0$. The metric tensor of the transverse space $g_{\perp, d-2}$ satisfies $g_{\perp, d-2}^{\mu \nu} p_{1, v}=g_{\perp, d-2}^{\mu \nu} p_{2, v}=0$ and $g_{\perp, d-2}^{\mu v} k_{\perp, v}=k_{\perp}^{\mu}$.

We write the four-momenta of the QCD partons as for the $q \bar{q} \rightarrow V$ process. We introduce $d$-dimensional vectors $t^{\mu}=(1, \mathbf{0})$ and $e_{3}^{\mu}=(0,0,0,1, \mathbf{0})$, and an additional vector $a^{\mu}$ that is orthogonal to both $t$ and $e_{3}$ and is normalized $a^{2}=-1$. We write the four-momenta in terms of $t, e_{3}$ and $a$ and obtain

$p_{1,2}=E_{1}\left(t \pm e_{3}\right), \quad p_{4}=E_{4}\left(t+\cos \theta_{41} e+\sin \theta_{41} a\right)$.

By comparing the two parametrizations of the vector $p_{4}$, we find

$\frac{k_{\perp}^{\mu} k_{\perp}^{\nu}}{k_{\perp}^{2}}=-a^{\mu} a^{v}$.

Since the transverse components of the gluon four-momentum decouple from the hard matrix element in the collinear limit, the vector $a^{\mu}$ only appears in the splitting function. We can 
then integrate the splitting function over the directions of the vector $a^{\mu}$ using

$$
\int \mathrm{d} \Omega_{d-2}^{(a)} a^{\mu} a^{v}=-\frac{g_{\perp, d-2}^{\mu \nu}}{d-2} \Omega_{d-2} .
$$

We find

$$
\begin{aligned}
& \int \frac{\mathrm{d} \Omega_{d-2}^{(a)}}{\Omega_{d-2}} P_{\mu \nu, g g}\left(z^{-1}\right) F_{\mathrm{LM}}^{\mu \nu}(z \cdot 1,2) \\
& =\left\langle P_{g g}\left(z^{-1}\right)\right\rangle F_{\mathrm{LM}}(z \cdot 1,2),
\end{aligned}
$$

where

$$
\left\langle P_{g g}(z)\right\rangle=2 C_{A}\left(\frac{1-z}{z}+\frac{z}{1-z}+z(1-z)\right)
$$

is the spin-averaged splitting function. It follows that averaging over the directions of the transverse components of the gluon momentum naturally appears in our construction at NLO; as a consequence, the rest of the NLO QCD calculation is identical to the $q \bar{q}$ case.

Before discussing spin correlations in the computation of NNLO QCD corrections, we note that, in the particular case of Higgs boson production, spin correlations are actually not needed. Indeed, the spin-correlated matrix element for $g g \rightarrow H$ is proportional to $g_{\perp, d-2}^{\mu v}$. As the result, the spinaveraged splitting function $P_{g g, \mu \nu} g_{\perp, d-2}^{\mu \nu}$ naturally appears in the calculation. We emphasize, however, that this feature is particular to the process $g g \rightarrow H$, so that understanding spin correlations is necessary in a more general context.

We then consider the generic NNLO case. Here, the situation is more complex since we have to consider the momenta of the two radiated gluons becoming collinear to the direction of the incoming partons. In the double-collinear partitions the situation is identical to the NLO case since the averaging over the transverse spaces of the two gluons is performed independently. The triple-collinear partitions require some discussion. We consider the case when collinear singularities arise because of the emissions of gluons $g_{4,5}$ off the gluon $g_{1}$. We parametrize the four-momenta of the final-state gluons as [30]

$$
\begin{aligned}
& p_{4}=E_{4}\left(t+\cos \theta_{4} e_{3}+\sin \theta_{4} a\right), \\
& p_{5}=E_{5}\left(t+\cos \theta_{5} e_{3}+\sin \theta_{5}\left(\cos \varphi_{45} a+\sin \varphi_{45} b\right)\right),
\end{aligned}
$$

where the vectors $t, a, e_{3}$ have already been defined in the discussion after Eq. (4.3) and the vector $b$ satisfies $t \cdot b=$ $e_{3} \cdot b=a \cdot e_{3}=0$, as well as $b^{2}=-1$.

We begin with the double-collinear limits that develop spin correlations. There are three possibilities: $g_{4}$ is collinear to $g_{5}, g_{5}$ is collinear to $g_{1}$ and $g_{4}$ is collinear to $g_{1}$. The first case is identical to the $q \bar{q} \rightarrow V$ process; it was discussed in Ref. [30] and we do not repeat this discussion here. The second case, $p_{5} \| p_{1}$, relevant for sector $(a)$, is discussed below. After that we comment on the third case, relevant for sector $(c)$.

Starting from the angular part of the phase space for sector (a) and considering the limit $x_{4} \rightarrow 0$, corresponding to $\theta_{51} \rightarrow 0$, we find

$$
\begin{aligned}
\lim _{x_{4} \rightarrow 0} \mathrm{~d} \Omega_{45}^{(a)}= & {\left[\frac{1}{8 \pi^{2}} \frac{(4 \pi)^{\epsilon}}{\Gamma(1-\epsilon)}\right]^{2}\left[\frac{\Gamma^{2}(1-\epsilon)}{\Gamma(1-2 \epsilon)}\right] } \\
& \times\left[\frac{\mathrm{d} \Omega_{d-2}^{(b)}}{\Omega_{d-2}} \frac{\mathrm{d} \Omega_{d-3}^{(a)}}{\Omega_{d-3}}\right] \frac{\mathrm{d} x_{3}}{x_{3}^{1+2 \epsilon}} \frac{\mathrm{d} x_{4}}{x_{4}^{1+\epsilon}} \\
& \times \frac{\mathrm{d} \lambda}{\pi[\lambda(1-\lambda)]^{1 / 2+\epsilon}}\left[128\left(1-x_{3}\right)\right]^{-\epsilon} 2 x_{3}^{2} x_{4},
\end{aligned}
$$

see Ref. [30]. Also, in this limit

$$
\sin \varphi=\sqrt{4 \lambda(1-\lambda)}, \quad \cos \varphi=-1+2 \lambda, \quad \lambda \in[0,1] .
$$

This follows immediately from the definition of $\sin \varphi$ and by inverting the definition of $\lambda$ in terms of $\cos \varphi$. We then rewrite

$$
\begin{aligned}
\lim _{x_{4} \rightarrow 0} \mathrm{~d} \Omega_{45}^{(a)}= & {\left[\frac{1}{8 \pi^{2}} \frac{(4 \pi)^{\epsilon}}{\Gamma(1-\epsilon)}\right]^{2}\left[\frac{\mathrm{d} \Omega_{d-2}^{(b)}}{\Omega_{d-2}} \frac{\mathrm{d} \Omega_{d-3}^{(a)}}{\Omega_{d-3}}\right] } \\
& \frac{2 \mathrm{~d} x_{3}}{\left[4 x_{3}\left(1-x_{3}\right)\right]^{\epsilon}} \frac{2 \mathrm{~d}\left(x_{3} x_{4} / 2\right)}{\left[4\left(x_{3} x_{4}\right) / 2\right]^{\epsilon}} \mathrm{d} \widetilde{\Lambda},
\end{aligned}
$$

with

$$
\begin{aligned}
\mathrm{d} \widetilde{\Lambda}= & {\left[16^{-\epsilon} \frac{\Gamma^{2}(1-\epsilon)}{\Gamma(1-2 \epsilon)} \frac{\mathrm{d} \lambda}{\pi[\lambda(1-\lambda)]^{1 / 2+\epsilon}}\right], } \\
& \int \mathrm{d} \tilde{\Lambda}=1 .
\end{aligned}
$$

We also have

$$
\begin{aligned}
& \int \mathrm{d} \tilde{\Lambda} \cos \varphi \sin \varphi=0, \\
& \int \mathrm{d} \widetilde{\Lambda} \cos ^{2} \varphi=\frac{1}{2(1-\epsilon)}, \quad \int \mathrm{d} \widetilde{\Lambda} \sin ^{2} \varphi=1-\frac{1}{2(1-\epsilon)} .
\end{aligned}
$$

These identities imply that in the $x_{4} \rightarrow 0$ limit

$$
\begin{aligned}
\left\langle\kappa_{5, \perp}^{\mu} \kappa_{5, \perp}^{\nu}\right\rangle= & \int \frac{\mathrm{d} \Omega_{d-3}^{(b)}}{\Omega_{d-3}} \mathrm{~d} \tilde{\Lambda}\left[a^{\mu} a^{\nu} \cos ^{2} \varphi_{45}\right. \\
& \left.+b^{\mu} b^{\nu} \sin ^{2} \varphi_{45}+\left(b^{\mu} a^{\nu}+a^{\mu} b^{\nu}\right) \cos \varphi_{45} \sin \varphi_{45}\right] \\
= & \frac{a^{\mu} a^{\nu}}{2(1-\epsilon)}+\frac{1-2 \epsilon}{2(1-\epsilon)} \int \frac{\mathrm{d} \Omega_{d-3}^{(b)}}{\Omega_{d-3}} b^{\mu} b^{\nu} \\
= & \frac{a^{\mu} a^{\nu}}{2(1-\epsilon)}+\frac{1-2 \epsilon}{2(1-\epsilon)} \frac{\left[-g_{\perp, d-3}^{\mu \nu}\right]}{d-3} \\
= & \frac{1}{2(1-\epsilon)}\left[a^{\mu} a^{\nu}-g_{\perp, d-3}^{\mu \nu}\right]=\frac{\left[-g_{\perp, d-2}^{\mu \nu}\right]}{2(1-\epsilon)} .
\end{aligned}
$$

Hence, in case of double-collinear limits with respect to the incoming partons, integration over the transverse directions of the collinear gluons always leads to spin-averaged 
splitting functions. This implies that the subsequent computational steps are conceptually identical to those of the $q \bar{q} \rightarrow V$ process. Finally, we note that the above discussion can be repeated verbatim for the limit $p_{4} \| p_{1}$, relevant for sector (c), if instead of Eq. (4.9) we use

$$
\begin{aligned}
& p_{4}=E_{4}\left(t+\cos \theta_{4} e_{3}+\sin \theta_{4}\left(\cos \varphi_{45} a+\sin \varphi_{45} b\right)\right), \\
& p_{5}=E_{5}\left(t+\cos \theta_{5} e_{3}+\sin \theta_{5} a\right) .
\end{aligned}
$$

It remains to discuss the triple-collinear limit that corresponds to the splitting $g_{1} \rightarrow g_{4}+g_{5}+g^{*}$. This splitting is described by the $P_{g g g}^{\mu \nu}$ splitting function that contains spin correlations, see e.g. Ref. [39]. This splitting function is a symmetric rank-two tensor constructed from $g_{\perp, d-2}^{\mu \nu}$, and the vectors $k_{4(5), \perp}^{\mu}$. These vectors read, c.f. Eq. (4.9),

$k_{4, \perp}^{\mu}=E_{4} \sin \theta_{4} a^{\mu}$,

$k_{5, \perp}^{\mu}=E_{5} \sin \theta_{5}\left(a^{\mu} \cos \varphi_{45}+b^{\mu} \sin \varphi_{45}\right)$.

In the triple-collinear limit, we need to integrate over the directions of the vectors $a$ and $b$. Note that the integration over the angle $\varphi_{45}$ is non-trivial since $2\left(p_{4} \cdot p_{5}\right)=s_{45}$ depends on it. To describe the integration of different tensor structures over the directions of $a$ and $b$, we introduce the notation

$$
\begin{aligned}
& \int \frac{\mathrm{d} \Omega_{d-2}^{(a)}}{\Omega_{d-2}} \frac{\mathrm{d} \Omega_{d-3}^{(b)}}{\Omega_{d-3}} X=\langle X\rangle_{a, b}, \\
& \int \frac{\mathrm{d} \Omega_{d-2}^{(a)}}{\Omega_{d-2}} X=\langle X\rangle_{a}, \quad \int \frac{\mathrm{d} \Omega_{d-3}^{(b)}}{\Omega_{d-3}} X=\langle X\rangle_{b} .
\end{aligned}
$$

We find the following results for the four tensor structures that contribute to $P_{g g g}^{\mu \nu}$

$$
\begin{aligned}
\left\langle g_{\perp, d-2}^{\mu \nu}\right\rangle= & g_{\perp, d-2}^{\mu \nu}, \\
\left\langle k_{4 \perp}^{\mu} k_{4 \perp}^{v}\right\rangle_{a, b}= & k_{4, \perp}^{2} \frac{g_{\perp, d-2}^{\mu \nu}}{d-2}, \\
\left\langle k_{5 \perp}^{\mu} k_{5 \perp}^{v}\right\rangle_{a, b}= & k_{5, \perp}^{2} \frac{g_{\perp, d-2}^{\mu \nu}}{d-2}, \\
\left\langle k_{4 \perp}^{\mu} k_{5 \perp}^{v}\right\rangle_{a, b}= & E_{4} \sin \theta_{4} E_{5} \sin \theta_{5} \\
& \times\left\langle a^{\mu}\left(a^{v} \cos \varphi_{45}+b^{\mu} \sin \varphi_{45}\right)\right\rangle_{a, b} \\
= & k_{4, \perp} \cdot k_{5, \perp} \frac{g_{\perp, d-2}^{\mu \nu}}{d-2} .
\end{aligned}
$$

We write the spin-correlated splitting function as

$$
\begin{aligned}
P_{g g g}^{\mu \nu}= & A_{1} g_{\perp, d-2}^{\mu \nu}+A_{2}\left(k_{4, \perp}^{\mu} k_{4, \perp}^{\nu}+k_{5, \perp}^{\mu} k_{5, \perp}^{v}\right) \\
& +A_{3}\left(k_{4, \perp}^{\mu} k_{5, \perp}^{\nu}+k_{4, \perp}^{\nu} k_{5, \perp}^{\mu}\right),
\end{aligned}
$$

and observe that Eq. (4.19) leads to

$$
\begin{aligned}
\left\langle P_{g g g}^{\mu v}\right\rangle_{a, b}= & g_{\perp, d-2}^{\mu \nu}\left(A_{1}+\frac{A_{2}}{d-2}\left(k_{4, \perp}^{2}+k_{5, \perp}^{2}\right)\right. \\
& \left.+\frac{A_{3}}{d-2}\left(2 k_{4, \perp} k_{5, \perp}\right)\right) .
\end{aligned}
$$

The same result is obtained upon replacing the spincorrelated splitting function by its spin-averaged version

$P_{g g g}^{\mu \nu} \rightarrow \frac{P_{g g g, \alpha \beta} g_{\perp, d-2}^{\alpha \beta}}{d-2} g_{\perp, d-2}^{\mu \nu}$.

Once this is done, the triple-collinear splittings in the $g g \rightarrow$ $H$ process can be treated in exactly the same way as in the Drell-Yan $q \bar{q} \rightarrow V$ case.

\subsection{Definition of partonic channels}

After discussing spin correlations, we proceed with setting up the NNLO QCD calculation for color-singlet production in gluon fusion. Starting from Eq. (2.1), we find it convenient to write the cross section for the generic process

$p p \rightarrow H+X$

as

$$
\begin{aligned}
\mathrm{d} \sigma_{f}^{\mathrm{H}}= & \int \mathrm{d} x_{1} \mathrm{~d} x_{2} g\left(x_{1}\right) g\left(x_{2}\right) \mathrm{d} \hat{\sigma}_{g g}^{\mathrm{H}} \\
& +\int \mathrm{d} x_{1} \mathrm{~d} x_{2} \sum_{\substack{a \in\left[-n_{f}, n_{f}\right] \\
a \neq 0}}\left[f_{a}\left(x_{1}\right) g\left(x_{2}\right) \mathrm{d} \hat{\sigma}_{q g}^{\mathrm{H}}\right. \\
& \left.+g\left(x_{1}\right) f_{a}\left(x_{2}\right) \mathrm{d} \hat{\sigma}_{g q}^{\mathrm{H}}\right] \\
& +\int \mathrm{d} x_{1} \mathrm{~d} x_{2} \sum_{\substack{a \in\left[-n_{f}, n_{f}\right] \\
a \neq 0}} f_{a}\left(x_{1}\right) f_{-a}\left(x_{2}\right) \mathrm{d} \hat{\sigma}_{q \bar{q}}^{\mathrm{H}} \\
& +\int \mathrm{d} x_{1} \mathrm{~d} x_{2} \sum_{\substack{a, b \in\left[-n_{f}, n_{f}\right] \\
a, b \neq 0, a \neq-b}} f_{a}\left(x_{1}\right) f_{b}\left(x_{2}\right) \mathrm{d} \hat{\sigma}_{q_{i} q_{j}}^{\mathrm{H}} .
\end{aligned}
$$

The first term is the $g g$ channel which is the only partonic channel contributing at LO. The terms on the second and third line correspond to the quark-gluon channels and quarkantiquark channel respectively, and enter at NLO. The $q_{i} q_{j}$ channel, where $q_{i}$ and $q_{j}$ can be (anti)quarks of either different or identical flavors, first appears at NNLO. We will discuss each of these channels separately in the following subsections. For simplicity, we will omit the "H" superscript from now on. We express our results in terms of fully renormalized amplitudes for the $p p \rightarrow H+X$ process, where $H$ is a generic color-singlet state. In the case of Higgs production in the $m_{t} \rightarrow \infty$ approximation, this implies that our 
results include both the divergent and the finite renormalization of the $H g g$ Wilson coefficient, see Appendix A for more details.

\subsection{LO and NLO}

At leading order, only the $g g$ channel contributes. We write

$2 s \cdot \mathrm{d} \hat{\sigma}_{g g}^{\mathrm{LO}}=\left\langle F_{\mathrm{LM}, g g}(1,2)\right\rangle$.

NLO corrections have a similar structure to those discussed in Sect. 3.1. The result for the $g g$ channel reads

$$
\begin{aligned}
2 s \cdot \mathrm{d} \hat{\sigma}_{g g}^{\mathrm{NLO}}= & \left\langle F_{\mathrm{LV}, g g}^{\mathrm{fin}}(1,2)+\frac{\alpha_{s}(\mu)}{2 \pi}\left[\frac{2 \pi^{2}}{3} C_{A}\right.\right. \\
& \left.\left.-2 \gamma_{g} \ln \left(\frac{\mu^{2}}{s}\right)\right] F_{\mathrm{LM}, g g}(1,2)\right\rangle \\
& +\frac{\alpha_{s}(\mu)}{2 \pi} \int_{0}^{1} \mathrm{~d} z\left[\mathcal{P}_{g g}^{\prime}(z)-\hat{P}_{g g, R}^{(0)} \ln \left(\frac{\mu^{2}}{s}\right)\right] \\
& \times\left\langle\frac{F_{\mathrm{LM}, g g}(z \cdot 1,2)+F_{\mathrm{LM}, g g}(1, z \cdot 2)}{z}\right\rangle \\
& +\left\langle\hat{\mathcal{O}}_{\mathrm{NLO}} F_{\mathrm{LM}, g g}(1,2,4)\right\rangle,
\end{aligned}
$$

with $\gamma_{g}=\beta_{0}=11 C_{A} / 6-n_{f} / 3$, and $F_{\mathrm{LV}, g g}^{\mathrm{fin}}$ and the various splitting functions are defined in Appendix $A$ and Appendix C, respectively.

NLO corrections to the $g q$ and $q g$ channel read

$$
\begin{aligned}
2 s \cdot \mathrm{d} \hat{\sigma}_{g q}^{\mathrm{NLO}}= & \frac{\alpha_{s}(\mu)}{2 \pi} \int_{0}^{1} \mathrm{~d} z\left\langle\frac{F_{\mathrm{LM}, g g}(1, z \cdot 2)}{z}\right\rangle \\
& \times\left[\mathcal{P}_{g q}^{\prime}(z)-\hat{P}_{g q, R}^{(0)} \ln \left(\frac{\mu^{2}}{s}\right)\right] \\
& +\left\langle\hat{\mathcal{O}}_{\mathrm{NLO}} F_{\mathrm{LM}, g q}(1,2,4)\right\rangle,
\end{aligned}
$$

and

$$
\begin{aligned}
2 s \cdot \mathrm{d} \hat{\sigma}_{q g}^{\mathrm{NLO}}= & \frac{\alpha_{s}(\mu)}{2 \pi} \int_{0}^{1} \mathrm{~d} z\left[\mathcal{P}_{g q}^{\prime}(z)-\hat{P}_{g q, R}^{(0)} \ln \left(\frac{\mu^{2}}{s}\right)\right] \\
& \times\left\langle\frac{F_{\mathrm{LM}, g g}(z \cdot 1,2)}{z}\right\rangle+\left\langle\hat{\mathcal{O}}_{\mathrm{NLO}} F_{\mathrm{LM}, q g}(1,2,4)\right\rangle,
\end{aligned}
$$

respectively. Finally, the $q \bar{q}$ channel starts contributing at NLO but it is finite at this order and can simply be written as

$2 s \cdot \mathrm{d} \hat{\sigma}_{q \bar{q}}^{\mathrm{NLO}}=\left\langle\hat{\mathcal{O}}_{\mathrm{NLO}} F_{\mathrm{LM}, q \bar{q}}(1,2,4)\right\rangle=\left\langle F_{\mathrm{LM}, q \bar{q}}(1,2,4)\right\rangle$.

\subsection{NNLO: gluon channel}

This channel has the same singularity structure as the DrellYan quark channels, cf. Sect. 3.2, and we use the same phasespace parametrization and partitioning described there. This means that the structure of the result is identical to what was discussed in Sect. 3.2, and we can write it as

$\mathrm{d} \hat{\sigma}_{g g}^{\mathrm{NNLO}}=\mathrm{d} \hat{\sigma}_{H+2, g g}^{\mathrm{NNLO}}+\mathrm{d} \hat{\sigma}_{H+1, g g}^{\mathrm{NNLO}}+\mathrm{d} \hat{\sigma}_{H, g g}^{\mathrm{NNLO}}$,

and

$$
\begin{aligned}
\mathrm{d} \hat{\sigma}_{H+2, g g}^{\mathrm{NNLO}}= & \mathrm{d} \hat{\sigma}_{1245, g g}^{\mathrm{NNLO},} \\
\mathrm{d} \hat{\sigma}_{H+1, g g}^{\mathrm{NNLO}}= & \mathrm{d} \hat{\sigma}_{124, g g}^{\mathrm{NNLO}}+\mathrm{d} \hat{\sigma}_{\mathrm{virt}_{124}, g g}^{\mathrm{NNLO}}, \\
\mathrm{d} \hat{\sigma}_{H, g g}^{\mathrm{NNLO}}= & \mathrm{d} \hat{\sigma}_{(z, \bar{z}), g}^{\mathrm{NNLO}}+\mathrm{d} \hat{\sigma}_{(1, z), g}^{\mathrm{NNLO}}+\mathrm{d} \hat{\sigma}_{(z, 2), g g}^{\mathrm{NNLO}} \\
& +\mathrm{d} \hat{\sigma}_{(1,2), g g}^{\mathrm{NNLO}}+\mathrm{d} \hat{\sigma}_{\text {virt }_{12}, g g}^{\mathrm{NNLO}} .
\end{aligned}
$$

We now list all these terms separately.

\section{Tree-level terms with NNLO kinematics}

$$
\begin{aligned}
& \mathrm{d} \hat{\sigma}_{1245, g g}^{\mathrm{NNLO}} \\
& =\sum_{(i j) \in d c}\left\langle\left[\left(I-C_{5 j}\right)\left(I-C_{4 i}\right)\right][I-\mathbb{S}]\left[I-S_{5}\right]\right. \\
& \left.\quad \times\left[d f_{4}\right]\left[d f_{5}\right] w^{4 i, 5 j} F_{\mathrm{LM}, g g}(1,2,4,5)\right\rangle \\
& \quad+\sum_{i \in t c}\left\langle\left[\theta^{(a)}\left[I-\mathbb{C}_{i}\right]\left[I-C_{5 i}\right]+\theta^{(b)}\left[I-\mathbb{C}_{i}\right]\left[I-C_{45}\right]\right.\right. \\
& \left.\quad+\theta^{(c)}\left[I-\mathbb{C}_{i}\right]\left[I-C_{4 i}\right]+\theta^{(d)}\left[I-\mathbb{C}_{i}\right]\left[I-C_{45}\right]\right] \\
& \left.\quad \times[I-\mathbb{S}]\left[I-S_{5}\right]\left[d f_{4}\right]\left[d f_{5}\right] w^{4 i, 5 i} F_{\mathrm{LM}, g g}(1,2,4,5)\right\rangle,
\end{aligned}
$$

which is analogous to Eq. (3.23).

\section{Tree-level terms with NLO kinematics}

$$
\begin{aligned}
\mathrm{d} \hat{\sigma}_{124, g g}^{\mathrm{NNLO}}=\frac{\alpha_{s}(\mu)}{2 \pi} \int_{0}^{1} \mathrm{~d} z\left\{\hat { P } _ { g g , R } ^ { ( 0 ) } ( z ) \left\langle\ln \frac{\rho_{41}}{4} \hat{\mathcal{O}}_{\mathrm{NLO}}\right.\right. \\
\times\left[\frac{\tilde{w}_{5 \| 1}^{41,51} F_{\mathrm{LM}, g g}(z \cdot 1,2,4)}{z}\right] \\
\left.+\ln \frac{\rho_{42}}{4} \hat{\mathcal{O}}_{\mathrm{NLO}}\left[\frac{\tilde{w}_{5 \| \mid 2}^{42,52} F_{\mathrm{LM}, g g}(1, z \cdot 2,4)}{z}\right]\right\rangle \\
+2 n_{f} \hat{P}_{q g, R}^{(0)}(z)\left\langle\ln \frac{\rho_{41}}{4} \hat{\mathcal{O}}_{\mathrm{NLO}}\right. \\
\quad \times\left[\frac{\tilde{w}_{5 \| 1}^{41,51} F_{\mathrm{LM}, q g}(z \cdot 1,2,4)}{z}\right] \\
\left.+\ln \frac{\rho_{42}}{4} \hat{\mathcal{O}}_{\mathrm{NLO}}\left[\frac{\tilde{w}_{5 \| \mid 2}^{42,52} F_{\mathrm{LM}, g q}(1, z \cdot 2,4)}{z}\right]\right\rangle \\
+\left[\mathcal{P}_{g g}^{\prime}(z)-\hat{P}_{g g, R}^{(0)}(z) \ln \left(\frac{\mu^{2}}{s}\right)\right]
\end{aligned}
$$




$$
\begin{aligned}
& \times\left\langle\hat{\mathcal{O}}_{\mathrm{NLO}}\left[\frac{F_{\mathrm{LM}, g g}(z \cdot 1,2,4)+F_{\mathrm{LM}, g g}(1, z \cdot 2,4)}{z}\right]\right\rangle \\
& +2 n_{f}\left[\mathcal{P}_{q g}^{\prime}(z)-\hat{P}_{q g, R}^{(0)}(z) \ln \left(\frac{\mu^{2}}{s}\right)\right] \\
& \left.\times\left\langle\hat{\mathcal{O}}_{\mathrm{NLO}}\left[\frac{F_{\mathrm{LM}, q g}(z \cdot 1,2,4)+F_{\mathrm{LM}, g q}(1, z \cdot 2,4)}{z}\right]\right\rangle\right\} \\
& +\frac{\alpha_{s}(\mu)}{2 \pi}\left\langle\hat { \mathcal { O } } _ { \mathrm { NLO } } \left[\Delta_{g} \cdot F_{\mathrm{LM}, g g}(1,2,4)\right.\right. \\
& \left.\left.+\Delta^{r} \cdot\left[r_{\mu} r_{v} F_{\mathrm{LM}, g g}^{\mu \nu}(1,2,4)\right]\right]\right\rangle,
\end{aligned}
$$

which is analogous to Eq. (3.48). The splitting functions in Eq. (4.33) are defined in Appendix $\mathrm{C}$, and $\Delta_{g}$ is given in Eq. (3.49) with $C_{g}=C_{A}$ and $\gamma_{g}=\mathcal{X}_{g}=\beta_{0}$.

3. Tree-level terms with $\mathrm{LO}$ kinematics involving $F_{\mathrm{LM}}(z$. $1, \bar{z} \cdot 2)$ :

$$
\begin{aligned}
\mathrm{d} \hat{\sigma}_{(z, \bar{z}), g g}^{\mathrm{NNLO}}= & \left(\frac{\alpha_{s}(\mu)}{2 \pi}\right)^{2} \int_{0}^{1} \mathrm{~d} z \mathrm{~d} \bar{z} \\
& \times\left[\mathcal{P}_{g g}^{\prime}(z)-\ln \left(\frac{\mu^{2}}{s}\right) \hat{P}_{g g, R}^{(0)}(z)\right] \\
& \times\left\langle\frac{F_{\mathrm{LM}, g g}(z \cdot 1, \bar{z} \cdot 2)}{z \bar{z}}\right\rangle \\
& \times\left[\mathcal{P}_{g g}^{\prime}(\bar{z})-\ln \left(\frac{\mu^{2}}{s}\right) \hat{P}_{g g, R}^{(0)}(\bar{z})\right] .
\end{aligned}
$$

4. Tree-level terms with LO kinematics involving $F_{\mathrm{LM}}(z$. $1,2)$ and $F_{\mathrm{LM}}(1, z \cdot 2)$

$$
\begin{aligned}
\mathrm{d} \hat{\sigma}_{(1, z), g g}^{\mathrm{NNLO}}+\mathrm{d} \hat{\sigma}_{(z, 2), g g}^{\mathrm{NNLO}} & \left(\frac{\alpha_{s}(\mu)}{2 \pi}\right)^{2} \int_{0}^{1} \mathrm{~d} z \mathcal{T}_{g g}(z) \\
& \times\left\langle\frac{F_{\mathrm{LM}, g g}(z \cdot 1,2)+F_{\mathrm{LM}, g g}(1, z \cdot 2)}{z}\right\rangle .
\end{aligned}
$$

5. Tree-level terms with LO kinematics involving $F_{\mathrm{LM}}(1,2)$ :

$$
\begin{aligned}
\mathrm{d} \hat{\sigma}_{(1,2), g g}^{\mathrm{NNO}}=\left\langle F_{\mathrm{LM}}(1,2)\right\rangle \times\left(\frac{\alpha_{s}(\mu)}{2 \pi}\right)^{2} \\
\quad \times\left\{C _ { A } ^ { 2 } \left[\frac{739}{81}+\frac{4}{3} \ln 2+\frac{187 \pi^{2}}{54}-2 \ln ^{2}(2)\right.\right. \\
\quad+\frac{11}{9} \pi^{2} \ln 2-\frac{407}{36} \zeta_{3}+\frac{13 \pi^{4}}{144}+\frac{\pi^{2}}{6} \ln ^{2} 2 \\
\quad-\frac{\ln ^{4} 2}{6}-\frac{7}{2} \zeta_{3} \ln 2-4 \mathrm{Li}_{4}\left(\frac{1}{2}\right) \\
-\ln \left(\frac{\mu^{2}}{s}\right)\left(\frac{37}{6}+\frac{11 \pi^{2}}{8}+23 \zeta_{3}\right)
\end{aligned}
$$

$$
\begin{aligned}
& \left.+\ln ^{2}\left(\frac{\mu^{2}}{s}\right)\left(\frac{121}{36}-\frac{2 \pi^{2}}{3}\right)\right] \\
& +C_{A} n_{f}\left[-\frac{214}{81}-\frac{227}{216} \pi^{2}-\frac{4}{3} \ln 2-\frac{2}{9} \pi^{2} \ln 2\right. \\
& +2 \ln ^{2} 2+\frac{37}{18} \zeta_{3}+\ln \left(\frac{\mu^{2}}{s}\right) \\
& \left.\times\left(\frac{94}{27}+\frac{\pi^{2}}{4}\right)-\frac{11}{9} \ln \left(\frac{\mu^{2}}{s}\right)^{2}\right] \\
& +n_{f}^{2}\left[\frac{11 \pi^{2}}{108}-\frac{10}{27} \ln \left(\frac{\mu^{2}}{s}\right)+\frac{1}{9} \ln ^{2}\left(\frac{\mu^{2}}{s}\right)\right] \\
& +\Theta_{b d}\left[C_{A}^{2}\left(-\frac{131}{36}+\frac{11}{3} \ln 2+\frac{\pi^{2}}{3}\right)\right. \\
& \left.\left.+C_{A} n_{f}\left(\frac{23}{36}-\frac{2}{3} \ln 2\right)\right]\right\} .
\end{aligned}
$$

6. Terms involving virtual corrections with NLO kinematics

$$
\mathrm{d} \hat{\sigma}_{\text {virt } 124, g g}^{\mathrm{NNLO}}=\left\langle\hat{\mathcal{O}}_{\mathrm{NLO}} F_{\mathrm{LV}, g g}^{\mathrm{fin}}(1,2,4)\right\rangle .
$$

\section{Terms involving virtual corrections with LO kinemat-} ics:

$$
\begin{aligned}
& \mathrm{d} \hat{\sigma}_{\mathrm{virt} 12, g g}^{\mathrm{NNLO}}=\left\langle F_{\mathrm{LVV}, g g}^{\mathrm{fin}}(1,2)+F_{\mathrm{LV}^{2}, g g}^{\mathrm{fin}}(1,2)\right. \\
& +\frac{\alpha_{s}(\mu)}{2 \pi}\left[\frac{2 \pi^{2}}{3} C_{A}-2 \gamma_{g} \ln \left(\frac{\mu^{2}}{s}\right)\right] \\
& \left.\quad \times F_{\mathrm{LV}, g g}^{\mathrm{fin}}(1,2)\right\rangle+\frac{\alpha_{s}(\mu)}{2 \pi} \int_{0}^{1} d z\left[\mathcal{P}_{g g}^{\prime}(z)\right. \\
& \left.-\ln \left(\frac{\mu^{2}}{s}\right) \hat{P}_{g g, R}^{(0)}(z)\right] \\
& \quad \times\left\langle\frac{F_{\mathrm{LV}, g g}^{\mathrm{fin}}(z \cdot 1,2)+F_{\mathrm{LV}, g g}^{\mathrm{fin}}(1, z \cdot 2)}{z}\right\rangle
\end{aligned}
$$

The above equations are analogous to Eqs. (3.56), (3.58), (3.62), (3.65) and (3.66) in Drell-Yan production, respectively. The finite remainders $F_{\mathrm{LVV}}^{\mathrm{fin}}, F_{\mathrm{LV}^{2}}^{\mathrm{fin}}$ and $F_{\mathrm{LV}}^{\mathrm{fin}}$ are defined in Appendix A, the finite remainder $F_{\mathrm{LV}}^{\mathrm{fin}}(1,2,4)$ is defined in Appendix B, the function $\mathcal{T}_{g g}$ is given in the ancillary file, and $\Theta_{b d}$ is given in Eqs. $(3.63,3.64)$.

\subsection{NNLO: quark-gluon channels}

The structure of this channel is analogous to the $q g$ channel for the Drell-Yan process, discussed in Sect. 3.3. We don't repeat the discussion here, and limit ourselves to presenting final results. To follow Sect. 3.3 as closely as possible, we 
focus on the $g \bar{q}$ channel. We write

$\mathrm{d} \hat{\sigma}_{g \bar{q}}^{\mathrm{NNLO}}=\mathrm{d} \hat{\sigma}_{H+2, g \bar{q}}^{\mathrm{NNLO}}+\mathrm{d} \hat{\sigma}_{H+1, g \bar{q}}^{\mathrm{NNLO}}+\mathrm{d} \hat{\sigma}_{H, g \bar{q}}^{\mathrm{NNLO}}$,

with

$$
\begin{aligned}
& \mathrm{d} \hat{\sigma}_{H+2, g \bar{q}}^{\mathrm{NNLO}}=\mathrm{d} \hat{\sigma}_{1245, g \bar{q}}^{\mathrm{NNLO}}, \\
& \mathrm{d} \hat{\sigma}_{H+1, g \bar{q}}^{\mathrm{NNLO}}=\mathrm{d} \hat{\sigma}_{124, g \bar{q}}^{\mathrm{NNLO}}+\mathrm{d} \hat{\sigma}_{\mathrm{virt}_{124}, g \bar{q}}^{\mathrm{NNLO}}, \\
& \mathrm{d} \hat{\sigma}_{H, g \bar{q}}^{\mathrm{NNLO}}=\mathrm{d} \hat{\sigma}_{(z, \bar{z}), g \bar{q}}^{\mathrm{NNLO}}+\mathrm{d} \hat{\sigma}_{(1, z), g \bar{q}}^{\mathrm{NNLO}}+\mathrm{d} \hat{\sigma}_{\text {virt }_{12}, g \bar{q}}^{\mathrm{NNLO}^{2}} .
\end{aligned}
$$

We display the individual contributions below.

\section{Tree-level terms with NNLO kinematics}

$$
\begin{aligned}
\mathrm{d} \hat{\sigma}_{1245, g \bar{q}}^{\mathrm{NNLO}} & =\sum_{(i j) \in d c}\left\langle\left[\left(I-C_{5 j}\right)\left(I-C_{4 i}\right)\right]\left[I-S_{5}\right]\right. \\
& \left.\times\left[d f_{4}\right]\left[d f_{5}\right] w^{4 i, 5 j} F_{\mathrm{LM}, g \bar{q}}(1,2,4,5)\right\rangle \\
& +\sum_{i \in t c} /\left[\theta^{(a)}\left[I-\boldsymbol{C}_{i}\right]\left[I-C_{5 i}\right]+\theta^{(b)}\left[I-\boldsymbol{C}_{i}\right]\left[I-C_{45}\right]\right. \\
& \left.+\theta^{(c)}\left[I-\mathbb{C}_{i}\right]\left[I-C_{4 i}\right]+\theta^{(d)}\left[I-\mathbb{C}_{i}\right]\left[I-C_{45}\right]\right] \\
& \left.\times\left[I-S_{5}\right]\left[d f_{4}\right]\left[d f_{5}\right] w^{4 i, 5 i} F_{\mathrm{LM}, g \bar{q}}(1,2,4,5)\right\rangle .
\end{aligned}
$$

\section{Tree-level terms with NLO kinematics}

$$
\begin{aligned}
& \mathrm{d} \hat{\sigma}_{124, g \bar{q}}^{\mathrm{NNLO}} \\
& =\frac{\alpha_{s}(\mu)}{2 \pi} \int_{0}^{1} \mathrm{~d} z\left\{\left\langle\operatorname { l n } \frac { \rho _ { 4 1 } } { 4 } \hat { \mathcal { O } } _ { \mathrm { NLO } } \left[\tilde{w}_{5 \| 1}^{41,51}\right.\right.\right. \\
& \left.\left.\times \frac{\hat{P}_{g g, R}^{(0)}(z) F_{\mathrm{LM}, g \bar{q}}(z \cdot 1,2,4)+\hat{P}_{q g, R}^{(0)} F_{\mathrm{LM}, q \bar{q}}(z \cdot 1,2,4)}{z}\right]\right) \\
& +\left\langle\ln \frac{\rho_{42}}{4} \hat{\mathcal{O}}_{\mathrm{NLO}}\right. \\
& \left.\times\left[\tilde{w}_{5, \mid 52}^{42,52} \frac{F_{\mathrm{LM}, g \bar{q}}(1, z \cdot 2,4) \hat{P}_{q q, R}^{(0)}(z)+F_{\mathrm{LM}, g g}(1, z \cdot 2,4) \hat{P}_{g q, R}^{(0)}(z)}{z}\right]\right) \\
& +\left[\mathcal{P}_{g g}^{\prime}(z)-\hat{P}_{g g, R}^{(0)}(z) \ln \left(\frac{\mu^{2}}{s}\right)\right]\left\langle\hat{\mathcal{O}}_{\mathrm{NLO}}\left[\frac{F_{\mathrm{LM}, g \bar{q}}(z \cdot 1,2,4)}{z}\right]\right\rangle \\
& +\left[\mathcal{P}_{q g}^{\prime}(z)-\hat{P}_{q g, R}^{(0)}(z) \ln \left(\frac{\mu^{2}}{s}\right)\right]\left\langle\hat{\mathcal{O}}_{\mathrm{NLO}}\left[\frac{F_{\mathrm{LM}, q \bar{q}}(z \cdot 1,2,4)}{z}\right]\right\rangle \\
& +\left\langle\hat{\mathcal{O}}_{\mathrm{NLO}}\left[\frac{F_{\mathrm{LM}, g \bar{q}}(1, z \cdot 2,4)}{z}\right]\left[\mathcal{P}_{q q}^{\prime}(z)-\hat{P}_{q q, R}^{(0)}(z) \ln \left(\frac{\mu^{2}}{s}\right)\right]\right\rangle \\
& \left.+\left\langle\hat{\mathcal{O}}_{\mathrm{NLO}}\left[\frac{F_{\mathrm{LM}, g g}(1, z \cdot 2,4)}{z}\right]\left[\mathcal{P}_{g q}^{\prime}(z)-\hat{P}_{g q, R}^{(0)}(z) \ln \left(\frac{\mu^{2}}{s}\right)\right]\right\rangle\right\} \\
& +\frac{\alpha_{s}(\mu)}{2 \pi}\left\langle\hat{\mathcal{O}}_{\mathrm{NLO}}\left[\Delta^{(q g)} \cdot F_{\mathrm{LM}, g q}(1,2,4)\right]\right\rangle \text {. }
\end{aligned}
$$

3. Tree-level terms with LO kinematics involving $F_{\mathrm{LM}}(z$. $1, \bar{z} \cdot 2)$ :

$$
\begin{aligned}
\mathrm{d} \hat{\sigma}_{(z, \bar{z}), g \bar{q}}^{\mathrm{NNLO}}=\left(\frac{\alpha_{s}(\mu)}{2 \pi}\right)^{2} \int_{0}^{1} \mathrm{~d} z \mathrm{~d} \bar{z}\left[\mathcal{P}_{g g}^{\prime}(z)\right. \\
\left.\quad-\ln \left(\frac{\mu^{2}}{s}\right) \hat{P}_{g g, R}^{(0)}(z)\right] \\
\quad \times\left\langle\frac{F_{\mathrm{LM}, g g}(z \cdot 1, \bar{z} \cdot 2)}{z \bar{z}}\right\rangle \\
\quad \times\left[\mathcal{P}_{g q}^{\prime}(\bar{z})-\ln \left(\frac{\mu^{2}}{s}\right) \hat{P}_{g q, R}^{(0)}(\bar{z})\right]
\end{aligned}
$$

4. Terms with LO kinematics involving $F_{\mathrm{LM}}(1, z \cdot 2)$ :

$$
\mathrm{d} \hat{\sigma}_{(1, z), g \bar{q}}^{\mathrm{NNLO}}=\left(\frac{\alpha_{s}(\mu)}{2 \pi}\right)^{2} \int_{0}^{1} \mathrm{~d} z \mathcal{T}_{g q}(z)\left\langle\frac{F_{\mathrm{LM}, g g}(1, z \cdot 2)}{z}\right\rangle .
$$

5. Terms involving virtual corrections with NLO kinematics:

$$
\mathrm{d} \hat{\sigma}_{\mathrm{virt}_{124}, g \bar{q}}^{\mathrm{NNLO}}=\left\langle\hat{\mathcal{O}}_{\mathrm{NLO}} F_{\mathrm{LV}, g \bar{q}}^{\mathrm{fin}}(1,2,4)\right\rangle .
$$

\section{Terms involving virtual corrections with LO kinemat-} ics:

$$
\begin{aligned}
\mathrm{d} \hat{\sigma}_{\text {virt }}^{\mathrm{NNO}, g \bar{q}}= & \frac{\alpha_{s}(\mu)}{2 \pi} \int_{0}^{1}\left[\mathcal{P}_{g q}^{\prime}(z)-\ln \left(\frac{\mu^{2}}{s}\right) \hat{P}_{g q, R}^{(0)}(z)\right] \\
& \times\left\langle\frac{F_{\mathrm{LV}, g g}^{\mathrm{fin}}(1, z \cdot 2)}{z}\right\rangle
\end{aligned}
$$

The splitting functions used in these equations are defined in Appendix $\mathrm{C}, \Delta^{(q g)}$ is given in Eq. (3.72), $\mathcal{T}_{g q}$ is given in the ancillary file, and the $F_{\mathrm{LV}}^{\mathrm{fin}}$ finite remainders are defined in Appendix A and B. Results for the $q g$ channel can be trivially obtained from the above formulas under a $1 \leftrightarrow 2$ replacement.

\subsection{NNLO: $q \bar{q}$ channel}

The singularity structure of this channel can be organized as follows. There are purely collinear singularities, coming from configurations where the $q$ and the $\bar{q}$ emit two $t$-channel gluons that produce a Higgs. These singularities have the same structure as those appearing in the $g g$ channel for the Drell-Yan process. Apart from these, there are other singular contributions which don't have a Drell-Yan equivalent. They stem from extra gluon emission in the $s$-channel $q \bar{q} \rightarrow H+g$ 
process. These are of NLO origin, so they don't pose any particular challenge.

Because of its simple singularity structure, we don't discuss this channel in detail. For completeness, we present final formulas that are obtained using the same setup that we employed for the $g g$ channel in Sect. 4.4. We write

$\mathrm{d} \hat{\sigma}_{q \bar{q}}^{\mathrm{NNLO}}=\mathrm{d} \hat{\sigma}_{H+2, q \bar{q}}^{\mathrm{NNLO}}+\mathrm{d} \hat{\sigma}_{H+1, q \bar{q}}^{\mathrm{NNLO}}+\mathrm{d} \hat{\sigma}_{H, q \bar{q}}^{\mathrm{NNLO}}$,

and

$$
\begin{aligned}
& \mathrm{d} \hat{\sigma}_{H+2, q \bar{q}}^{\mathrm{NNLO}}=\mathrm{d} \hat{\sigma}_{1245, q \bar{q}}^{\mathrm{NNLO}}, \\
& \mathrm{d} \hat{\sigma}_{H+1, q \bar{q}}^{\mathrm{NNLO}}=\mathrm{d} \hat{\sigma}_{124, q \bar{q}}^{\mathrm{NNLO}}+\mathrm{d} \hat{\sigma}_{\text {virt }{ }_{124}, q \bar{q}}^{\mathrm{NNLO}}, \\
& \mathrm{d} \hat{\sigma}_{H, q \bar{q}}^{\mathrm{NNO}}=\mathrm{d} \hat{\sigma}_{(z, \bar{z}), q \bar{q}}^{\mathrm{NNLO}} .
\end{aligned}
$$

We now present the individual contributions:

\section{Tree-level terms with NNLO kinematics}

$$
\begin{aligned}
\mathrm{d} \hat{\sigma}_{1245, q \bar{q}}^{\mathrm{NNLO}} & =\sum_{(i j) \in d c}\left\langle\left[\left(I-C_{5 j}\right)\left(I-C_{4 i}\right)\right][I-S S]\left[I-S_{5}\right]\right. \\
& \left.\times\left[d f_{4}\right]\left[d f_{5}\right] w^{4 i, 5 j} F_{\mathrm{LM}, q \bar{q}}(1,2,4,5)\right\rangle \\
& +\sum_{i \in t c}\left\langle\left[\theta^{(a)}\left[I-\mathbb{C}_{i}\right]\left[I-C_{5 i}\right]\right.\right. \\
& +\theta^{(b)}\left[I-\mathbb{C}_{i}\right]\left[I-C_{45}\right] \\
& +\theta^{(c)}\left[I-\mathbb{C}_{i}\right]\left[I-C_{4 i}\right] \\
& \left.+\theta^{(d)}\left[I-\mathbb{C}_{i}\right]\left[I-C_{45}\right]\right] \\
& \times[I-S S]\left[I-S_{5}\right]\left[d f_{4}\right]\left[d f_{5}\right] w^{4 i, 5 i} \\
& \left.\times F_{\mathrm{LM}, q \bar{q}}(1,2,4,5)\right\rangle .
\end{aligned}
$$

\section{Tree-level terms with NLO kinematics}

$$
\begin{aligned}
\mathrm{d} \hat{\sigma}_{124, q \bar{q}}^{\mathrm{NNLO}}=\frac{\alpha_{s}(\mu)}{2 \pi} \int_{0}^{1} \mathrm{~d} z\left\{\hat{P}_{q q, R}^{(0)}(z)\right. \\
\quad \times\left\langle\ln \frac{\rho_{41}}{4} \hat{\mathcal{O}}_{\mathrm{NLO}}\left[\frac{\tilde{w}_{5 \| \mid 11}^{41,51} F_{\mathrm{LM}, q \bar{q}}(z \cdot 1,2,4)}{z}\right]\right. \\
\left.\quad+\ln \frac{\rho_{42}}{4} \hat{\mathcal{O}}_{\mathrm{NLO}}\left[\frac{\tilde{w}_{5 \| \mid 2}^{42,52} F_{\mathrm{LM}, q \bar{q}}(1, z \cdot 2,4)}{z}\right]\right\rangle \\
+\hat{P}_{g q, R}^{(0)}(z)\left\langle\ln \frac{\rho_{41}}{4} \hat{\mathcal{O}}_{\mathrm{NLO}}\right. \\
\quad \times\left[\frac{\tilde{w}_{5|| 1}^{41,51} F_{\mathrm{LM}, g \bar{q}}(z \cdot 1,2,4)}{z}\right] \\
\left.\quad+\ln \frac{\rho_{42}}{4} \hat{\mathcal{O}}_{\mathrm{NLO}}\left[\frac{\tilde{w}_{5 \| \mid 2}^{42,52} F_{\mathrm{LM}, q g}(1, z \cdot 2,4)}{z}\right]\right\rangle
\end{aligned}
$$

$$
\begin{aligned}
& +\left[\mathcal{P}_{q q}^{\prime}(z)-\hat{P}_{q q, R}^{(0)}(z) \ln \left(\frac{\mu^{2}}{s}\right)\right] \\
& \times\left\langle\hat{\mathcal{O}}_{\mathrm{NLO}}\left[\frac{F_{\mathrm{LM}, q \bar{q}}(z \cdot 1,2,4)+F_{\mathrm{LM}, q \bar{q}}(1, z \cdot 2,4)}{z}\right]\right\rangle \\
& +\left[\mathcal{P}_{g q}^{\prime}(z)-\hat{P}_{g q, R}^{(0)}(z) \ln \left(\frac{\mu^{2}}{s}\right)\right] \\
& \left.\times\left\langle\hat{\mathcal{O}}_{\mathrm{NLO}}\left[\frac{F_{\mathrm{LM}, g \bar{q}}(z \cdot 1,2,4)+F_{\mathrm{LM}, q g}(1, z \cdot 2,4)}{z}\right]\right\rangle\right\} \\
& +\frac{\alpha_{s}(\mu)}{2 \pi}\left\langle\hat { \mathcal { O } } _ { \mathrm { NLO } } \left[\Delta_{q} \cdot F_{\mathrm{LM}, q \bar{q}}(1,2,4)\right.\right. \\
& \left.\left.+\Delta^{r} \cdot\left[r_{\mu} r_{v} F_{\mathrm{LM}, q \bar{q}}^{\mu \nu}(1,2,4)\right]\right]\right\rangle .
\end{aligned}
$$

3. Tree-level terms with $\mathrm{LO}$ kinematics involving $F_{\mathrm{LM}}(z$. $1, \bar{z} \cdot 2)$ :

$$
\begin{aligned}
\mathrm{d} \hat{\sigma}_{(z, \bar{z}), q \bar{q}}^{\mathrm{NNLO}}= & \left(\frac{\alpha_{s}(\mu)}{2 \pi}\right)^{2} \int_{0}^{1} \mathrm{~d} z \mathrm{~d} \bar{z} \\
& \times\left[\mathcal{P}_{g q}^{\prime}(z)-\ln \left(\frac{\mu^{2}}{s}\right) \hat{P}_{g q, R}^{(0)}(z)\right] \\
& \times\left(\frac{F_{\mathrm{LM}, g g}(z \cdot 1, \bar{z} \cdot 2)}{z \bar{z}}\right) \\
& \times\left[\mathcal{P}_{g q}^{\prime}(\bar{z})-\ln \left(\frac{\mu^{2}}{s}\right) \hat{P}_{g q, R}^{(0)}(\bar{z})\right] .
\end{aligned}
$$

\section{Terms involving virtual corrections with NLO kine- matics:}

$$
\mathrm{d} \hat{\sigma}_{\text {virt }_{124}, q \bar{q}}^{\mathrm{NNLO}}=\left\langle F_{\mathrm{LV}, q \bar{q}}^{\mathrm{fin}}(1,2,4)\right\rangle .
$$

The splitting functions used in these equations are defined in Appendix C, $\Delta_{q}$ is given in Eq. (3.49) with $C_{i}=C_{F}, \gamma_{q}=$ $3 C_{F} / 2, \mathcal{X}_{q}=3 C_{A} / 2$, and the $F_{\mathrm{LV}}^{\mathrm{fin}}$ finite remainder is defined in Appendix B. Note that, contrary to all the cases discussed so far, $F_{\mathrm{LV}, q \bar{q}}^{\mathrm{fin}}$ does not require any additional regularization.

\subsection{NNLO: quark channels}

The singularity structure of this channel is the same as that of the $g g$ channel for the Drell-Yan process. Because of this, we use the same parametrization described in Sect. 3.4. We write

$\mathrm{d} \hat{\sigma}_{q_{i} q_{j}}^{\mathrm{NNLO}}=\mathrm{d} \hat{\sigma}_{H+2, q_{i} q_{j}}^{\mathrm{NNLO}}+\mathrm{d} \hat{\sigma}_{H+1, q_{i} q_{j}}^{\mathrm{NNLO}}+\mathrm{d} \hat{\sigma}_{H, q_{i} q_{j}}^{\mathrm{NNLO}}$,

with

$$
\begin{aligned}
& \mathrm{d} \hat{\sigma}_{H+2, q_{i} q_{j}}^{\mathrm{NNLO}}=\mathrm{d} \hat{\sigma}_{1245, q_{i} q_{j}}^{\mathrm{NNLO}}, \\
& \mathrm{d} \hat{\sigma}_{H+1, q_{i} q_{j}}^{\mathrm{NNLO}}=\mathrm{d} \hat{\sigma}_{124, q_{i} q_{j}}^{\mathrm{NNLO}}, \\
& \mathrm{d} \hat{\sigma}_{H, q_{i} q_{j}}^{\mathrm{NNLO}}=\mathrm{d} \hat{\sigma}_{(z, \bar{z}), q_{i} q_{j}}^{\mathrm{NNLO} .}
\end{aligned}
$$


Repeating the steps discussed in Sect. 3.4 we obtain the following results.

\section{Tree-level terms with NNLO kinematics}

$$
\begin{aligned}
\mathrm{d} \hat{\sigma}_{1245, q_{i} q_{j}}^{\mathrm{NNLO}} & \\
= & \left\langle\left[ I-C_{41}-C_{42}-C_{51}-C_{52}\right.\right. \\
& \left.+C_{42} C_{51}+C_{41} C_{52}\right] \\
& \left.\times\left[d f_{4}\right]\left[d f_{5}\right] F_{\mathrm{LM}, q_{i} q_{j}}(1,2,4,5)\right\rangle .
\end{aligned}
$$

\section{Tree-level terms with NLO kinematics}

$$
\begin{aligned}
\mathrm{d} \hat{\sigma}_{124, q_{i} q_{j}}^{\mathrm{NNLO}} & =\frac{\alpha_{s}(\mu)}{2 \pi} \int_{0}^{1} \mathrm{~d} z\left[\mathcal{P}_{g q}^{\prime}(z)-\hat{P}_{g q, R}^{(0)}(z) \ln \left(\frac{\mu^{2}}{s}\right)\right] \\
& \times\left\langle\hat{\mathcal{O}}_{\mathrm{NLO}}\left[\frac{F_{\mathrm{LM}, g q_{j}}(z \cdot 1,2,4)+F_{\mathrm{LM}, q_{i} g}(1, z \cdot 2,4)}{z}\right]\right\rangle .
\end{aligned}
$$

3. Tree-level terms with LO kinematics involving $F_{\mathrm{LM}}(z$. $1, \bar{z} \cdot 2)$ :

$$
\begin{aligned}
\mathrm{d} \hat{\sigma}_{(z, \bar{z}), q_{i} q_{j}}^{\mathrm{NNLO}}= & \left(\frac{\alpha_{s}(\mu)}{2 \pi}\right)^{2} \int_{0}^{1} \mathrm{~d} z \mathrm{~d} \bar{z} \\
& \times\left[\mathcal{P}_{g q}^{\prime}(z)-\ln \left(\frac{\mu^{2}}{s}\right) \hat{P}_{g q}^{(0)}(z)\right] \\
& \times\left\langle\frac{F_{\mathrm{LM}, g g}(z \cdot 1, \bar{z} \cdot 2)}{z \bar{z}}\right\rangle \\
& \times\left[\mathcal{P}_{g q}^{\prime}(\bar{z})-\ln \left(\frac{\mu^{2}}{s}\right) \hat{P}_{g q}^{(0)}(\bar{z})\right]
\end{aligned}
$$

\section{Validation of results}

In this section, we describe the numerical checks that have been used to validate the results described in the preceding sections. We use the processes $p p \rightarrow Z$ and $p p \rightarrow H$ as test cases, since for both of these processes the NNLO QCD corrections to the inclusive cross sections are known analytically $[34,35]$. This allows us to perform a high-precision check of our formulas.

We begin by describing our setup. We consider protonproton collisions with $13 \mathrm{TeV}$ center-of-mass energy. We use $m_{Z}=91.1876 \mathrm{GeV}, m_{H}=125 \mathrm{GeV}$ and $m_{t}=$ 173.2 $\mathrm{GeV}$ for the $Z$, the Higgs and the top quark masses, respectively. We derive the weak coupling constant from $g_{W}^{2}=4 \sqrt{2} m_{W}^{2} G_{F}$, with $m_{W}=80.398 \mathrm{GeV}$ and $G_{F}=$
$1.16639 \times 10^{-5} \mathrm{GeV}^{-2}$. The weak mixing angle is computed from $\sin ^{2} \theta_{W}=1-m_{W}^{2} / m_{Z}^{2}$. We will consider both on-shell $p p \rightarrow Z$ production and $p p \rightarrow e^{+} e^{-}$production. In the latter case, we include both the $Z$ and the $\gamma^{*}$ contributions, and we use $\Gamma_{Z}=2.4952 \mathrm{GeV}$.

For the Higgs case, we consider $p p \rightarrow H$ production in the $m_{t} \rightarrow \infty$ approximation and describe the Higgs coupling to gluons using the effective interaction Lagrangian $\mathcal{L}_{I}=-\lambda_{H g g} H G_{\mu \nu}^{(a)} G^{\mu \nu,(a)}$, see Appendix A for details. The coupling $\lambda_{H g g}$ depends on the Higgs vacuum expectation value $v$. For our results, we use $v^{2}=\left(G_{F} \sqrt{2}\right)^{-1}$. All computations are done using the NNPDF3.0 parton distribution set [40], with 5 active flavors. We employ LO/NLO/NNLO sets for LO/NLO/NNLO predictions, respectively. We use the value of the strong coupling and its evolution provided by the PDF sets, with $\alpha_{s}\left(m_{Z}\right)=0.118$ at $(\mathrm{N}) \mathrm{NLO}$, and $\alpha_{s}\left(m_{Z}\right)=0.130$ at LO.

We first consider fully inclusive on-shell $Z$ production. We compare results obtained within our framework to the analytic results of Ref. [34] that we implemented in HOPPET [41]. We study each partonic channel individually, and additionally split some of the channels into contributions with different color factors in order to validate all the different singularity structures independently. We show results for a single fixed factorization and renormalization scale $\mu=2 m_{Z}$, although we have performed the same check for different scales as well. We note that these inputs are not chosen for their phenomenological relevance, but rather to provide stringent checks on our results.

We summarize our findings in Table 1. It shows that our framework allows for extremely high precision results, with numerical errors at the per mille level or better. ${ }^{9}$ These results are always fully compatible with the analytic ones within the numerical uncertainties. We remind the reader that these numbers refer to the NNLO coefficients, which implies absolute precision on the physical cross section. Analogous results for the case of Higgs production for equal renormalization and factorization scales $\mu_{R}=\mu_{F}=m_{H} / 2$ are shown in Table 2. Again, we find it convenient to perform numerical checks for different partonic channels independently. Also in this case, our numerical results have tiny uncertainties and are in perfect agreement with the analytic values obtained from Ref. [35].

Having fully validated our results, we now briefly investigate the performance of the framework when applied to the computation of physically relevant predictions. Specifically, we explore the computational effort required to obtain predictions for physical quantities at the per mille level. We start by considering inclusive Higgs production, at the 13 TeV LHC. For this study, we set $\mu_{R}=\mu_{F}=m_{H}$. Running

\footnotetext{
9 The larger error in some channels is caused by non-negligible cancellations between different contributions to the final result.
} 
Table 1 Different contributions to the NNLO coefficient for on-shell $Z$ production at the 13 TeV LHC with $\mu_{R}=\mu_{F}=$ $2 m_{Z}$. All the color factors are included in the numerical results. The residual Monte-Carlo integration error is shown in brackets. See text for details

\begin{tabular}{llll}
\hline Channel & Color structures & Numerical result (nb) & Analytic result (nb) \\
\hline$q_{i} \bar{q}_{i} \rightarrow g g$ & - & $8.351(1)$ & 8.3516 \\
$q_{i} \bar{q}_{i} \rightarrow q_{j} \bar{q}_{j}$ & $C_{F} T_{R} n_{\text {up }}, C_{F} T_{R} n_{\mathrm{dn}}$ & $-2.1378(5)$ & -2.1382 \\
& $C_{F}\left(C_{A}-2 C_{F}\right)$ & $-4.8048(3) \times 10^{-2}$ & $-4.8048 \times 10^{-2}$ \\
& $C_{F} T_{R}$ & $5.441(7) \times 10^{-2}$ & $5.438 \times 10^{-2}$ \\
$q_{i} q_{j} \rightarrow q_{i} q_{j} \quad(i \neq-j)$ & $C_{F} T_{R}$ & $0.4182(5)$ & 0.4180 \\
& $C_{F}\left(C_{A}-2 C_{F}\right)$ & $-9.26(1) \times 10^{-4}$ & $-9.26 \times 10^{-4}$ \\
$q_{i} g+g q_{i}$ & - & $-9.002(9)$ & -8.999 \\
$g g$ & - & $1.0772(1)$ & 1.0773 \\
\hline
\end{tabular}

Table 2 Different contributions to the NNLO coefficient for on-shell $H$ production at the $13 \mathrm{TeV}$ LHC with $\mu_{R}=\mu_{F}=m_{H} / 2$. The residual Monte-Carlo integration error is shown in brackets. The labels $q q$ and $q q^{\prime}$ refer to quark initial states with identical and different flavors, respectively. See text for details

\begin{tabular}{lll}
\hline Channel & Numerical result $(\mathrm{pb})$ & Analytic result $(\mathrm{pb})$ \\
\hline$g g \rightarrow g g$ & $9.397(1)$ & 9.398 \\
$g g \rightarrow q \bar{q}$ & $-1.243(2)$ & -1.243 \\
$q g+g q$ & $0.7865(8)$ & 0.7861 \\
$q \bar{q}$ & $1.145(1) \cdot 10^{-2}$ & $1.146 \cdot 10^{-2}$ \\
$q q$ & $2.139(3) \cdot 10^{-2}$ & $2.140 \cdot 10^{-2}$ \\
$q q^{\prime}$ & $5.967(5) \cdot 10^{-2}$ & $5.970 \cdot 10^{-2}$ \\
\hline
\end{tabular}

for less than an hour on a single core of a standard laptop, we obtain

$$
\begin{aligned}
\sigma_{\mathrm{H}}^{\mathrm{LO}} & =17.03(0) \mathrm{pb} ; \quad \sigma_{\mathrm{H}}^{\mathrm{NLO}}=30.25(1) \mathrm{pb} ; \\
\sigma_{\mathrm{H}}^{\mathrm{NNLO}} & =39.96(2) \mathrm{pb} .
\end{aligned}
$$

As one can see from Eq. (5.1) the numerical uncertainty on the full NNLO cross section is below one per mille. The result is in full agreement ${ }^{10}$ with the benchmark predictions reported in Ref. [42].

We now move to fiducial cross sections. We consider $p p \rightarrow Z / \gamma^{*} \rightarrow e^{-} e^{+}$production in the fiducial volume defined by symmetric lepton cuts, as studied in Ref. [42]. Specifically, we require that the transverse momentum and rapidity of each lepton satisfy

$$
p_{T, \ell}>25 \mathrm{GeV} \quad\left|\eta_{\ell}\right|<2.47 \text {, }
$$

and that the invariant mass of the lepton pair is in a window $66 \mathrm{GeV}<m_{e^{-} e^{+}}<116 \mathrm{GeV}$. In this case, we use $\mu_{R}=$ $\mu_{F}=m_{Z}$. Running on a single core of a standard laptop for about an hour, we obtain

$$
\begin{aligned}
\sigma_{\mathrm{DY}}^{\mathrm{LO}} & =650.4 \pm 0.1 \mathrm{pb} ; \quad \sigma_{\mathrm{DY}}^{\mathrm{NLO}}=700.2 \pm 0.3 \mathrm{pb} ; \\
\sigma_{\mathrm{DY}}^{\mathrm{NNLO}} & =734.8 \pm 1.4 \mathrm{pb} .
\end{aligned}
$$

10 The different LO cross section w.r.t. Ref. [42] is due to a different choice of LO PDFs.
We note that in this case the error is at the few per mille level. We compared the NNLO $K$-factor against the benchmark result presented in Ref. [42], and found agreement within the numerical precision.

As a final comment, we note that although the processes studied here are very simple, which makes it difficult to predict how the framework will perform for more complicated ones, these results are very encouraging.

\section{Conclusions}

In this paper, we presented compact analytic formulas that describe the fully-differential production of color-singlet final states in hadron collisions. We studied final states that, at leading order, can be produced either in $q \bar{q}$ or in $g g$ annihilation.

Our calculation employs the nested soft-collinear subtraction scheme that we developed earlier in Ref. [30]. However, compared to its original formulation, we found it useful to modify it to allow for a simpler analytic integration of the triple-collinear limits. We explained the required changes in Sect. 2.

We validated our results by using them to numerically compute NNLO QCD contributions to the total cross sections of $Z$ and $H$ production in proton collisions, and comparing them with results based on the convolution of known analytic results for the partonic cross sections for these two processes with parton distribution functions. For both $Z$ and $H$ production, we found an agreement at a better-than-per-mille level for NNLO coefficient functions. We also showed that our computation can deal with fiducial cuts quite efficiently.

As far as we know, the results presented in this paper provide the first implementation of a fully local and fully analytic NNLO QCD subtraction scheme. Although - as we already emphasized in the introduction - it is difficult to say to what extent these nice features of the subtraction scheme will help with its efficiency, we hope that they will be helpful in that respect. However, regardless of the performance issues, the physical clarity and overall transparency of the obtained for- 
mulas give us hope that analytic, local NNLO QCD subtractions for arbitrarily complex hadron collider processes are within reach.

Acknowledgements We are grateful to Mainz Institute for Theoretical Physics for hospitality extended to us during the work on this paper. R.R. is also grateful to the Galileo Galilei Institute for hospitality and support. We thank B. Mistlberger for providing computer-readable results for the NNLO corrections to the Higgs inclusive cross section. The research of K.M. is supported by BMBF Grant $05 \mathrm{H} 18 \mathrm{VKCC} 1$ and by the Deutsche Forschungsgemeinschaft (DFG, German Research Foundation) under Grant 396021762 - TRR 257.

Data Availability Statement This manuscript has no associated data or the data will not be deposited. [Authors' comment: This is a theoretical study and no experimental data has been listed.]

Open Access This article is distributed under the terms of the Creative Commons Attribution 4.0 International License (http://creativecomm ons.org/licenses/by/4.0/), which permits unrestricted use, distribution, and reproduction in any medium, provided you give appropriate credit to the original author(s) and the source, provide a link to the Creative Commons license, and indicate if changes were made.

Funded by SCOAP ${ }^{3}$.

\section{A Purely virtual contribution: definitions}

We consider the UV-renormalized amplitude for the process $p_{1}+p_{2} \rightarrow V$

$$
\begin{aligned}
\mathcal{A}\left(p_{1}, p_{2} ;\left\{p_{V}\right\}\right)= & \mathcal{A}_{0}(1,2)+\frac{\alpha_{s}(\mu)}{2 \pi} \mathcal{A}_{1}(1,2) \\
& +\left(\frac{\alpha_{s}(\mu)}{2 \pi}\right)^{2} \mathcal{A}_{2}(1,2)+\cdots .
\end{aligned}
$$

Following Ref. [43], we write

$$
\begin{aligned}
& \mathcal{A}_{1}(1,2)=\mathcal{I}_{1}(\epsilon) \mathcal{A}_{0}(1,2)+\mathcal{A}_{1, \text { fin }}(1,2), \\
& \mathcal{A}_{2}(1,2)=\mathcal{I}_{2}(\epsilon) \mathcal{A}_{0}(1,2)+\mathcal{I}_{1}(\epsilon) \mathcal{A}_{1}(1,2)+\mathcal{A}_{2, \text { fin }}(1,2),
\end{aligned}
$$

with $\mathcal{A}_{i \text {,fin }}$ finite in four dimensions. The explicit form of the $\mathcal{I}_{i}$ operators read [43]

$$
\begin{aligned}
\mathcal{I}_{1}(\epsilon) & =-\frac{e^{\epsilon \gamma_{E}}}{\Gamma(1-\epsilon)}\left(\frac{C_{i}}{\epsilon^{2}}+\frac{\gamma_{i}}{\epsilon}\right) e^{i \pi \epsilon}\left(\frac{\mu^{2}}{s_{12}}\right)^{\epsilon}, \\
\mathcal{I}_{2}(\epsilon) & =-\frac{1}{2} \mathcal{I}_{1}^{2}(\epsilon)-\frac{\beta_{0}}{\epsilon} \mathcal{I}_{1}(\epsilon)+\frac{e^{-\epsilon \gamma_{E}} \Gamma(1-2 \epsilon)}{\Gamma(1-\epsilon)} \\
\times & \left(\frac{\beta_{0}}{\epsilon}+K\right) \mathcal{I}_{1}(2 \epsilon)+\frac{e^{\epsilon \gamma_{E}}}{\Gamma(1-\epsilon)} \frac{H_{i}}{2 \epsilon}
\end{aligned}
$$

with

$$
\beta_{0}=\frac{11}{6} C_{A}-\frac{2}{3} T_{R} n_{f}, \quad K=\left(\frac{67}{18}-\frac{\pi^{2}}{6}\right) C_{A}-\frac{10}{9} T_{R} n_{f} .
$$

For $q \bar{q} \rightarrow V$ reactions, $i=q$ and

$$
\begin{aligned}
C_{q}= & C_{F}, \quad \gamma_{q}=\frac{3}{2} C_{F}, \\
H_{q}= & C_{F}^{2}\left(\frac{\pi^{2}}{2}-6 \zeta_{3}-\frac{3}{8}\right) \\
& +C_{A} C_{F}\left(\frac{245}{216}-\frac{23}{48} \pi^{2}+\frac{13}{2} \zeta_{3}\right) \\
& +C_{F} n_{f}\left(\frac{\pi^{2}}{24}-\frac{25}{108}\right),
\end{aligned}
$$

while for $g g \rightarrow V$ reactions $i=g$ and

$$
\begin{aligned}
C_{g}= & C_{A}, \quad \gamma_{g}=\beta_{0}, \\
H_{g}= & C_{A}^{2}\left(\frac{5}{12}+\frac{11}{144} \pi^{2}+\frac{\zeta_{3}}{2}\right)+C_{A} n_{f}\left(-\frac{29}{27}-\frac{\pi^{2}}{72}\right) \\
& +\frac{C_{F} n_{f}}{2}+\frac{5}{27} n_{f}^{2} .
\end{aligned}
$$

To express the virtual contribution to the cross-section, it is also useful to define

$$
\begin{aligned}
I_{12}(\epsilon) & =2 \operatorname{Re}\left[\mathcal{I}_{1}(\epsilon)\right] \\
& =-2 \cos (\epsilon \pi) \frac{e^{\epsilon \gamma_{E}}}{\Gamma(1-\epsilon)}\left(\frac{\mu^{2}}{s_{12}}\right)^{\epsilon}\left[\frac{C_{i}}{\epsilon^{2}}+\frac{\gamma_{i}}{\epsilon}\right] .
\end{aligned}
$$

The NLO virtual correction can then be written as

$$
\begin{aligned}
2 s \cdot \mathrm{d} \hat{\sigma}^{\mathrm{NLO}, \mathrm{V}}= & \left(\frac{\alpha_{s}(\mu)}{2 \pi}\right) I_{12}(\epsilon)\left\langle F_{L M}(1,2)\right\rangle \\
& +\left\langle F_{L V, \text { fin }}(1,2)\right\rangle,
\end{aligned}
$$

with $F_{L V \text {,fin }}$ finite and proportional to $2 \operatorname{Re}\left[\mathcal{A}_{0} \mathcal{A}_{1, \text { fin }}^{*}\right]$. Similarly, we can write the purely virtual corrections at NNLO as

$$
\begin{aligned}
2 s \cdot & \mathrm{d} \hat{\sigma}^{\mathrm{NNLO}, \mathrm{VV}} \\
= & {\left[\frac{I_{12}^{2}(\epsilon)}{2}-\frac{\beta_{0}}{\epsilon} I_{12}(\epsilon)+\frac{e^{-\epsilon \gamma_{E}} \Gamma(1-2 \epsilon)}{\Gamma(1-\epsilon)}\right.} \\
& \left.\times\left(\frac{\beta_{0}}{\epsilon}+K\right) I_{12}(2 \epsilon)+\frac{e^{\epsilon \gamma_{E}}}{\Gamma(1-\epsilon)} \frac{H_{i}}{\epsilon}\right] \\
& \times\left(\frac{\alpha_{s}(\mu)}{2 \pi}\right)^{2}\left\langle F_{\mathrm{LM}}(1,2)\right\rangle+I_{12}(\epsilon)\left(\frac{\alpha_{s}(\mu)}{2 \pi}\right) \\
& \times\left\langle F_{\mathrm{LV}}^{\mathrm{fin}}(1,2)\right\rangle+\left\langle F_{\mathrm{LVV}}^{\mathrm{fin}}(1,2)\right\rangle+\left\langle F_{\mathrm{LV}^{2}}^{\mathrm{fin}}(1,2)\right\rangle,
\end{aligned}
$$

with $F_{\mathrm{LVV}}^{\mathrm{fin}}$ and $F_{\mathrm{LV}^{2}}^{\mathrm{fin}}$ finite and proportional to $2 \operatorname{Re}\left[\mathcal{A}_{0} \mathcal{A}_{2, \text { fin }}^{*}\right]$ and $\left|\mathcal{A}_{1, \text { fin }}\right|^{2}$, respectively.

\section{A.1 Finite remainder: Drell-Yan}

In this section, we report the finite remainders for the DrellYan process, see e.g. [44]. We obtain

$$
\left.\left\langle F_{\mathrm{LV}, f_{a} f_{b}}^{\mathrm{fin}}(1,2)\right\rangle\right|_{\mu^{2}=Q^{2}}
$$




$$
\begin{aligned}
&=-8 C_{F}\left(\frac{\alpha_{s}}{2 \pi}\right)\left\langle F_{\mathrm{LM}, f_{a} f_{b}}(1,2)\right\rangle+\mathcal{O}(\epsilon), \\
&\left.\left\langle F_{\mathrm{LV}^{2}, f_{a} f_{b}}^{\mathrm{fin}}(1,2)\right\rangle\right|_{\mu^{2}=Q^{2}}=16 C_{F}^{2}\left(\frac{\alpha_{s}}{2 \pi}\right)^{2}\left\langle F_{\mathrm{LM}, f_{a} f_{b}}(1,2)\right\rangle+\mathcal{O}(\epsilon), \\
&\left.\left\langle F_{\mathrm{LVV}, f_{a} f_{b}}^{\mathrm{fin}}(1,2)\right\rangle\right|_{\mu^{2}=Q^{2}} \\
&=\left(\frac{\alpha_{s}}{2 \pi}\right)^{2}\left[C_{F}^{2}\left(\frac{255}{16}+\frac{29 \pi^{2}}{12}-15 \zeta_{3}-\frac{11 \pi^{4}}{90}\right)\right. \\
& \quad+C_{F} C_{A}\left(-\frac{51157}{1296}-\frac{107 \pi^{2}}{72}+\frac{659 \zeta_{3}}{36}+\frac{31 \pi^{4}}{240}\right) \\
&\left.\quad+C_{F} n_{f}\left(\frac{4085}{648}+\frac{7 \pi^{2}}{36}-\frac{\zeta_{3}}{18}\right)\right] \\
& \quad \times\left\langle F_{\mathrm{LM}, f_{a} f_{b}}(1,2)\right\rangle+\mathcal{O}(\epsilon),
\end{aligned}
$$

with $Q^{2}=p_{V}^{2}$ and $\alpha_{s}=\alpha_{s}(Q)$. Results for generic $\mu$ can easily be obtained from renormalization group evolution (RGE) arguments.

\section{A.2 Finite remainder: Higgs}

In this section, we report the finite remainders for the Higgs process. More precisely, we consider a theory where the Higgs is coupled directly to gluons through the effective interaction Lagrangian

$\mathcal{L}_{I}=-\lambda_{H g g} H G_{\mu \nu}^{(a)} G^{\mu \nu,(a)}$,

where the (bare) $\mathrm{Hgg}$ coupling is given by

$\lambda_{H g g, b}=-\frac{\alpha_{s}}{12 \pi v} C\left(\alpha_{S}\right) Z_{\mathrm{eff}}\left(\alpha_{s}\right)$.

In this formula, $\alpha_{s}=\alpha_{s}(\mu)$ is the renormalized coupling in a theory with 5 light flavors, $v$ is the Higgs v.e.v. and the divergent $\left(Z_{\text {eff }}\left(\alpha_{s}\right)\right)$ and finite $\left(C\left(\alpha_{s}\right)\right)$ parts of the Wilson coefficient renormalization are given in the $\overline{\mathrm{MS}}$ scheme by

$$
\begin{aligned}
Z_{\text {eff }}\left(\alpha_{s}\right)= & 1-\frac{\beta_{0}}{\epsilon}\left(\frac{\alpha_{s}}{2 \pi}\right)+\left[\frac{\beta_{0}^{2}}{\epsilon^{2}}-\frac{\beta_{1}}{\epsilon}\right]\left(\frac{\alpha_{s}}{2 \pi}\right)^{2}+\mathcal{O}\left(\alpha_{s}^{3}\right), \\
C\left(\alpha_{s}\right)= & 1+\left[\frac{5}{2} C_{A}-\frac{3}{2} C_{F}\right]\left(\frac{\alpha_{s}}{2 \pi}\right) \\
& +\left[\frac{1063}{144} C_{A}^{2}-\frac{25}{3} C_{A} C_{F}+\frac{27}{8} C_{F}^{2}\right. \\
& -\frac{47}{72} C_{A} n_{f}-\frac{5}{8} C_{F} n_{f}-\frac{5}{48} C_{A}-\frac{C_{F}}{6} \\
& \left.+\ln \left(\frac{\mu^{2}}{m_{t}^{2}}\right)\left(\frac{7}{4} C_{A}^{2}-\frac{11}{4} C_{A} C_{F}+C_{F} n_{f}\right)\right] \\
& \times\left(\frac{\alpha_{s}}{2 \pi}\right)^{2}+\mathcal{O}\left(\alpha_{s}^{3}\right),
\end{aligned}
$$

see e.g. [45]. In Eq. (A.14), $m_{t}$ is the top-quark mass, $\beta_{0}$ has been defined in Eq. (A.4) and

$\beta_{1}=\frac{17}{6} C_{A}^{2}-\frac{5}{3} C_{A} T_{R} n_{f}-C_{F} T_{R} n_{f}$.

Combining the result for the $\mathrm{Hgg}$ form factor in e.g. [44] with the finite part of the Wilson coefficient renormalization, we obtain for the Higgs finite remainders

$$
\begin{aligned}
& \left.\left\langle F_{\mathrm{LV}, g g}^{\mathrm{fin}}(1,2)\right\rangle\right|_{\mu^{2}=Q^{2}} \\
& =\left(\frac{\alpha_{s}}{2 \pi}\right)\left[5 C_{A}-3 C_{F}\right]\left\langle F_{\mathrm{LM}, g g}(1,2)\right\rangle+\mathcal{O}(\epsilon), \\
& \left.\left\langle F_{L V^{2}, g g, \mathrm{fin}}(1,2)\right\rangle\right|_{\mu^{2}=Q^{2}} \\
& =\left(\frac{\alpha_{s}}{2 \pi}\right)^{2}\left[\beta_{0}^{2} \pi^{2}+\left(\frac{5}{2} C_{A}-\frac{3}{2} C_{F}\right)^{2}\right] \\
& \quad \times\left\langle F_{\mathrm{LM}, g g}(1,2)\right\rangle+\mathcal{O}(\epsilon), \\
& \left.\left\langle F_{\mathrm{LVV}, g g}^{\mathrm{fin}}(1,2)\right\rangle\right|_{\mu^{2}=Q^{2}}=\left(\frac{\alpha_{s}}{2 \pi}\right)^{2} \\
& \quad \times\left\{C_{A}^{2}\left(\frac{5105}{324}-\frac{17 \pi^{2}}{3}-\frac{253 \zeta_{3}}{36}+\frac{\pi^{4}}{144}\right)\right. \\
& \quad+C_{A} n_{f}\left(-\frac{458}{81}+\frac{481 \pi^{2}}{216}-\frac{49 \zeta_{3}}{18}\right) \\
& \quad+C_{F} n_{f}\left(-\frac{67}{12}+4 \zeta_{3}\right)-\frac{23}{108} n_{f}^{2} \pi^{2} \\
& \quad+\left[\frac{1063}{72} C_{A}^{2}+\frac{27}{4} C_{F}^{2}-\frac{50}{3} C_{A} C_{F}-\frac{47}{36} C_{A} n_{f}\right. \\
& \quad-\frac{5}{4} C_{F} n_{f}-\frac{5}{24} C_{A}-\frac{1}{3} C_{F} \\
& \left.\left.\quad+\left(\frac{7}{2} C_{A}^{2}-\frac{11}{2} C_{A} C_{F}+2 C_{F} n_{f}\right) \ln \left(\frac{Q^{2}}{m_{t}^{2}}\right)\right]\right\} \\
& \quad \times\left\langle F_{\mathrm{LM}, g g}(1,2)\right\rangle+\mathcal{O}(\epsilon),
\end{aligned}
$$

with $Q^{2}=p_{H}^{2}$ and $\alpha_{s}=\alpha_{s}(Q)$. Results for generic $\mu$ can easily be obtained from RGE arguments.

\section{B Real-virtual contribution: definitions}

We consider the one-loop amplitude for the process

$f_{1}+f_{2} \rightarrow V / H+f_{4}$,

where $V / H$ indicates either Drell-Yan or Higgs production, as in Sects. 3 and 4. Following Appendix A, we write it as

$\mathcal{A}\left(p_{1}, p_{2}, p_{4} ;\left\{p_{V}\right\}\right)=\mathcal{A}_{0}(1,2,4)$

$$
+\frac{\alpha_{s}(\mu)}{2 \pi} \mathcal{A}_{1}(1,2,4)+\cdots,
$$


with

$\mathcal{A}_{1}(1,2,4)=\mathcal{I}_{1}(1,2,4 ; \epsilon) \mathcal{A}_{0}(1,2,4)+\mathcal{A}_{1, \mathrm{fin}}(1,2,4)$.

In the above, $\mathcal{I}_{1}(1,2,4 ; \epsilon)$ is the equivalent of $\mathcal{I}_{1}(\epsilon)$ of Eq. (A.2) for the $f_{1}+f_{2} \rightarrow V / H+f_{4}$ kinematic configurations (cf. Ref. [43]), and $\mathcal{A}_{1, \text { fin }}(1,2,4)$ is finite. We then define

$$
I_{124}(\epsilon)=2 \operatorname{Re}\left[\mathcal{I}_{1}(1,2,4 ; \epsilon)\right],
$$

and write the real-virtual contribution to the NNLO crosssection as

$$
\begin{aligned}
\mathrm{d} \hat{\sigma}^{\mathrm{NNLO}, \mathrm{RV}}= & \frac{\alpha_{s}(\mu)}{2 \pi}\left\langle I_{124}(\epsilon) F_{\mathrm{LM}}(1,2,4)\right\rangle \\
& +\left\langle F_{\mathrm{LV}}^{\mathrm{fin}}(1,2,4)\right\rangle,
\end{aligned}
$$

with $F_{\mathrm{LV}, i j}^{\mathrm{fin}}(1,2,4)$ finite. The explicit form of $I_{124}$ depends on the color structure of the process. Since the process Eq. (B.1) must involve either a gluon and a $q \bar{q}$ pair or 3 gluons, we can classify the most general case according to the position of a gluon and write

$$
\begin{aligned}
I_{1_{i} 2_{j} 4_{g}}= & \frac{e^{\epsilon \gamma_{E}}}{\Gamma(1-\epsilon)}\left\{\cos (\epsilon \pi)\left(\frac{\mu^{2}}{s_{12}}\right)^{\epsilon}\right. \\
& \times\left[\frac{C_{A}-2 C_{i}}{\epsilon^{2}}+\frac{\mathcal{X}_{i}-2 \gamma_{i}}{\epsilon}\right] \\
& \left.-\left[\left(\frac{\mu^{2}}{s_{14}}\right)^{\epsilon}+\left(\frac{\mu^{2}}{s_{24}}\right)^{\epsilon}\right]\left[\frac{C_{A}}{\epsilon^{2}}+\frac{\mathcal{X}_{i}+\gamma_{g}}{2 \epsilon}\right]\right\}, \\
I_{1_{g} 2_{i} 4_{j}}= & \frac{e^{\epsilon \gamma_{E}}}{\Gamma(1-\epsilon)}\left\{\left(\frac{\mu^{2}}{s_{24}}\right)^{\epsilon}\left[\frac{C_{A}-2 C_{i}}{\epsilon^{2}}+\frac{\mathcal{X}_{i}-2 \gamma_{i}}{\epsilon}\right]\right. \\
& -\left[\left(\frac{\mu^{2}}{s_{14}}\right)^{\epsilon}+\cos (\epsilon \pi)\left(\frac{\mu^{2}}{s_{12}}\right)^{\epsilon}\right] \\
& \left.\times\left[\frac{C_{A}}{\epsilon^{2}}+\frac{\mathcal{X}_{i}+\gamma_{g}}{2 \epsilon}\right]\right\}, \\
I_{1_{i} 2_{g} 4_{j}}= & \frac{e^{\epsilon \gamma_{E}}}{\Gamma(1-\epsilon)}\left\{\left(\frac{\mu^{2}}{s_{14}}\right)^{\epsilon}\left[\frac{C_{A}-2 C_{i}}{\epsilon^{2}}+\frac{\mathcal{X}_{i}-2 \gamma_{i}}{\epsilon}\right]\right. \\
& -\left[\left(\frac{\mu^{2}}{s_{24}}\right)^{\epsilon}+\cos (\epsilon \pi)\left(\frac{\mu^{2}}{s_{12}}\right)^{\epsilon}\right] \\
& \left.\times\left[\frac{C_{A}}{\epsilon^{2}}+\frac{\mathcal{X}_{i}+\gamma_{g}}{2 \epsilon}\right]\right\},
\end{aligned}
$$

with

$\mathcal{X}_{q}=\frac{3}{2} C_{A}, \quad \mathcal{X}_{g}=\gamma_{g}$.

Here $s_{i j}=2 E_{i} E_{j} \rho_{i j}$, with $E_{i, j}>0$.

\section{Splitting functions}

In this appendix we collect the relevant splitting functions used in the main text. We write the LO Altarelli-Parisi splitting functions as

$\hat{P}_{i j}^{(0)}(z)=\hat{P}_{i j, R}^{(0)}(z)+\hat{P}_{i j, \delta}^{(0)} \delta(1-z)$,

with

$$
\begin{aligned}
& \hat{P}_{q q, R}^{(0)}(z)=C_{F}\left[\frac{2}{(1-z)_{+}}-(1+z)\right], \\
& \hat{P}_{q g, R}^{(0)}(z)=T_{R}\left[z^{2}+(1-z)^{2}\right], \\
& \hat{P}_{g q, R}^{(0)}(z)=C_{F}\left[\frac{1+(1-z)^{2}}{z}\right], \\
& \hat{P}_{g g, R}^{(0)}(z)=2 C_{A}\left[\frac{1}{(1-z)_{+}}+\frac{1}{z}+z(1-z)-2\right],
\end{aligned}
$$

and

$$
\begin{aligned}
\hat{P}_{q q, \delta}^{(0)} & =\gamma_{q}=\frac{3}{2} C_{F}, \quad \hat{P}_{g g, \delta}^{(0)}=\gamma_{g}=\beta_{0} \\
& =\frac{11}{6} C_{A}-\frac{2}{3} T_{R} n_{f}, \quad \hat{P}_{q g, \delta}^{(0)}=\hat{P}_{g q, \delta}^{(0)}=0 .
\end{aligned}
$$

The $\mathcal{P}_{i j}^{\prime}$ splitting functions are related to the $\mathcal{O}(\epsilon)$ part of the LO splitting functions, and read

$$
\begin{aligned}
\mathcal{P}_{q q}^{\prime}(z)= & C_{F}\left(4\left[\frac{\ln (1-z)}{1-z}\right]_{+}\right. \\
& -2(1+z) \ln (1-z)+(1-z)), \\
\mathcal{P}_{q g}^{\prime}(z)= & T_{R}\left(2\left[z^{2}+(1-z)^{2}\right] \ln (1-z)+2 z(1-z)\right), \\
\mathcal{P}_{g q}^{\prime}(z)= & C_{F}\left(2\left[\frac{1+(1-z)^{2}}{z}\right] \ln (1-z)+z\right), \\
\mathcal{P}_{g g}^{\prime}(z)= & 2 C_{A}\left(2\left[\frac{\ln (1-z)}{1-z}\right]_{+}\right. \\
& \left.+2\left[\frac{1}{z}+z(1-z)-2\right] \ln (1-z)\right) .
\end{aligned}
$$

\section{Transition functions}

The transition functions $\mathcal{T}_{i j}$ introduced in the text are generalizations of NLO Altarelli-Parisi splitting functions. They depend on the scale $\mu$ of the process (though this dependence is of course entirely determined by RGE arguments), on an energy fraction $z$ and, in general, on the choice of partition functions through the factor

$\Theta_{a c} \equiv-\left\langle\left[I-C_{41}\right]\left[\frac{\rho_{12}}{\rho_{41} \rho_{42}}\right]\left(\tilde{w}_{5|| 1}^{41,51} \ln \frac{\rho_{41}}{4}\right)\right\rangle$.

For the choice Eq. (3.22), we obtain

$\Theta_{a c}=1+\ln 2$. 
We now show results for a typical transition function, namely the nonsinglet function relevant for Drell-Yan production. It reads.

$$
\begin{aligned}
& \mathcal{T}_{q q}^{\mathrm{NS}}=C_{F}^{2}\left[4 \tilde{\mathcal{D}}_{1}(z)\right] \Theta_{a c}+C_{F}^{2}\left[8 \tilde{\mathcal{D}}_{3}(z)\right. \\
& +16 \tilde{\mathcal{D}}_{0}(z) \zeta(3)+\frac{(z+1)}{12} \ln ^{3}(z) \\
& +\frac{3\left(2 z^{2}+2 z-7\right) \ln ^{2}(z)}{4(1-z)}+(4(z+1) \ln (z+1) \\
& \left.+\frac{22 z^{2}+5 z-17}{2(1-z)}\right) \ln (z)-\frac{\pi^{2}(8-5 z) z}{3(1-z)}+\frac{(23 z-17)}{2} \\
& -\ln 2(1-z)\left(2(1-z)+\frac{\left(1-7 z^{2}\right) \ln (z)}{2(1-z)}\right)+\ln (1-z) \\
& \times\left(\frac{19 z}{2}+\frac{\pi^{2}\left(3-5 z^{2}\right)}{6(1-z)}+\frac{\left(7 z^{2}-2 z+7\right) \ln (z)}{1-z}-10\right) \\
& +4(z+1) \operatorname{Li}_{2}(-z)-\left(\frac{2\left(2 z^{2}-5\right)}{1-z}+\frac{\left(3-5 z^{2}\right) \ln (1-z)}{1-z}\right)
\end{aligned}
$$$$
\mathrm{Li}_{2}(z)+\left[\frac{1+z^{2}}{1-z}\right] \times\left(-\frac{5}{2} \ln (1-z) \ln ^{2}(z)-4 \mathrm{Li}_{2}(-z) \ln (z)\right.
$$$$
\left.-\mathrm{Li}_{2}(z) \ln (z)+\frac{2}{3} \pi^{2} \ln (z)+8 \mathrm{Li}_{3}(-z)\right)
$$$$
\left.+\frac{\left(9 z^{2}+1\right) \operatorname{Li}_{3}(1-z)}{1-z}-\frac{\left(1-3 z^{2}\right) \operatorname{Li}_{3}(z)}{1-z}+\frac{\left(3 z^{2}+7\right) \zeta_{3}}{1-z}\right]
$$$$
+C_{F} n_{f}\left[\frac{4}{3} \tilde{\mathcal{D}}_{2}(z)-\frac{20}{9} \tilde{\mathcal{D}}_{1}(z)+\tilde{\mathcal{D}}_{0}(z)\left(\frac{34}{27}-\frac{\pi^{2}}{3}+\frac{2 \ln 2}{3}\right)\right.
$$$$
+\frac{(5 z-11)}{18}-\frac{1}{3}(1-z)[2 \ln (1-z)+\ln 2]
$$$$
+\frac{\left(5 z^{2}+6 z-7\right) \ln (z)}{18(1-z)}+\left[\frac{1+z^{2}}{1-z}\right]
$$$$
\left.\times\left(-\frac{1}{4} \ln ^{2}(z)+\frac{2 \operatorname{Li}_{2}(z)}{3}-\frac{\pi^{2}}{9}\right)\right]
$$$$
+C_{A} C_{F}\left[-\frac{22}{3} \tilde{\mathcal{D}}_{2}(z)+\left(\frac{134}{9}-\frac{2 \pi^{2}}{3}\right) \tilde{\mathcal{D}}_{1}(z)\right.
$$$$
-\left(\frac{208}{27}-\frac{11 \pi^{2}}{6}+\frac{2 \ln 2}{3}-9 \zeta_{3}\right) \tilde{\mathcal{D}}_{0}(z)
$$$$
+\frac{\left(7 z^{2}-12 z+27\right) \ln ^{2}(z)}{8(1-z)}-\left(\frac{83 z^{2}+114 z-109}{18(1-z)}\right.
$$$$
+2(z+1) \ln (z+1)) \ln (z)-\frac{2}{9}(17 z-5)
$$$$
+\frac{\pi^{2}\left(19 z^{2}-6 z+31\right)}{36(1-z)}+\ln (1-z)
$$

$$
\begin{aligned}
& \times\left(\frac{58-55 z}{6}-(1-z) \ln (z)\right) \\
& -2(z+1) \mathrm{Li}_{2}(-z)-\frac{2\left(4 z^{2}-3 z+10\right) \mathrm{Li}_{2}(z)}{3(1-z)} \\
& +\left[\frac{1+z^{2}}{1-z}\right]\left(\frac{7 \ln ^{3}(z)}{12}-\ln ^{2}(1-z) \ln (z)+2 \operatorname{Li}_{2}(-z) \ln (z)\right. \\
& +\frac{\pi^{2}}{3} \ln (z)+\frac{\pi^{2}}{6} \ln (1-z)+[\ln (z)-\ln (1-z)] \operatorname{Li}_{2}(z) \\
& \left.-5 \mathrm{Li}_{3}(1-z)-4 \mathrm{Li}_{3}(-z)-4 \mathrm{Li}_{3}(z)+\zeta_{3}\right) \\
& \left.+\frac{1}{3}(1-z) \ln 2\right]+\ln \left(\frac{\mu^{2}}{s}\right)\left\{\beta _ { 0 } C _ { F } \left(4 \tilde{\mathcal{D}}_{1}(z)\right.\right. \\
& \left.-\frac{10}{3} \tilde{\mathcal{D}}_{0}(z)-\frac{\left(1+z^{2}\right) \ln (z)}{1-z}+z-1\right) \\
& +C_{A} C_{F}\left[\left(\frac{\pi^{2}}{3}-\frac{4}{3}\right) \tilde{\mathcal{D}}_{0}(z)-\frac{\left(1+z^{2}\right) \ln ^{2}(z)}{2(1-z)}\right. \\
& -3(1-z)-(z+1) \ln (z)]+C_{F}^{2}\left[-12 \tilde{\mathcal{D}}_{2}(z)-12 \tilde{\mathcal{D}}_{1}(z)\right. \\
& -2(z+1) \operatorname{Li}_{2}(1-z)+\frac{\left(3 z^{2}+1\right) \ln ^{2}(z)}{2(1-z)} \\
& -\frac{\left(4 z^{2}+2 z-3\right) \ln (z)}{1-z}+2 \ln (1-z) \\
& \left.\left.\left(\frac{2 z^{2} \ln (z)}{1-z}-z+1\right)+2(1-z)\right]\right\} \\
& +\ln ^{2}\left(\frac{\mu^{2}}{s}\right)\left\{C _ { F } ^ { 2 } \left(4 \tilde{\mathcal{D}}_{1}(z)+6 \tilde{\mathcal{D}}_{0}(z)\right.\right. \\
& \left.\left.-\frac{\left(3 z^{2}+1\right) \ln (z)}{2(1-z)}+z-1\right)-C_{F} \beta_{0} \tilde{\mathcal{D}}_{0}(z)\right\} \text {, }
\end{aligned}
$$

where we have defined

$\tilde{\mathcal{D}}_{i}(z) \equiv\left[\frac{\ln ^{i}(1-z)}{1-z}\right]_{+}-\frac{1}{2}(1+z) \ln ^{i}(1-z)$,

and as usual $\beta_{0}=11 C_{A} / 6-n_{f} / 3$. Expressions for all the other relevant transition functions have the same form as Eq. (D.3), and can be found in the ancillary file.

\section{References}

1. A. Gehrmann-De Ridder, T. Gehrmann, E.W.N. Glover, JHEP 0509, 056 (2005)

2. A. Gehrmann-De Ridder, T. Gehrmann, E.W.N. Glover, Phys. Lett. B 612, 49 (2005)

3. A. Gehrmann-De Ridder, T. Gehrmann, E.W.N. Glover, Phys. Lett. B 612, 36 (2005) 
4. A. Daleo, T. Gehrmann, D. Maitre, JHEP 0704, 016 (2007)

5. A. Daleo, A. Gehrmann-De Ridder, T. Gehrmann, G. Luisoni, JHEP 1001, 118 (2010)

6. T. Gehrmann, P.F. Monni, JHEP 1112, 049 (2011)

7. R. Boughezal, A. Gehrmann-De Ridder, M. Ritzmann, JHEP 1102, 098 (2011)

8. A. Gehrmann-De Ridder, T. Gehrmann, M. Ritzmann, JHEP 1210, 047 (2012)

9. J. Currie, E.W.N. Glover, S. Wells, JHEP 1304, 066 (2013)

10. M. Czakon, Phys. Lett. B 693, 259 (2010)

11. M. Czakon, Nucl. Phys. B 849, 250 (2011)

12. M. Czakon, D. Heymes, Nucl. Phys. B 890, 152 (2014)

13. R. Boughezal, K. Melnikov, F. Petriello, Phys. Rev. D 85, 034025 (2012)

14. M. Cacciari, F.A. Dreyer, A. Karlberg, G.P. Salam, G. Zanderighi, Phys. Rev. Lett. 115(8), 082002 (2015)

15. S. Catani, M. Grazzini, Phys. Rev. Lett. 98, 222002 (2007)

16. M. Grazzini, JHEP 0802, 043 (2008)

17. R. Boughezal, C. Focke, X. Liu, F. Petriello, Phys. Rev. Lett. 115(6), 062002 (2015)

18. J. Gaunt, M. Stahlhofen, F.J. Tackmann, J.R. Walsh, JHEP 09, 058 (2015)

19. V. Del Duca, C. Duhr, A. Kardos, G. Somogyi, Z. Trocsanyi, Phys. Rev. Lett. 117(15), 152004 (2016)

20. V. Del Duca, C. Duhr, A. Kardos, G. Somogyi, Z. Szor, Z. Trocsanyi, Z. Tulipant, Phys. Rev. D 94(7), 074019 (2016)

21. I. Moult, L. Rothen, I.W. Stewart, F.J. Tackmann, H.X. Zhu, Phys. Rev. D 95(7), 074023 (2017)

22. I. Moult, L. Rothen, I.W. Stewart, F.J. Tackmann, H.X. Zhu, Phys. Rev. D 97(1), 014013 (2018)

23. M.A. Ebert, I. Moult, I.W. Stewart, F.J. Tackmann, G. Vita, H.X. Zhu, JHEP 1812, 084 (2018)
24. R. Boughezal, X. Liu, F. Petriello, JHEP 1703, 160 (2017)

25. R. Boughezal, A. Isgro, F. Petriello, Phys. Rev. D 97(7), 076006 (2018)

26. M.A. Ebert, I. Moult, I.W. Stewart, F.J. Tackmann, G. Vita, H.X. Zhu, JHEP 1904, 123 (2019). arXiv:1812.08189 [hep-ph]

27. L. Magnea, E. Maina, G. Pelliccioli, C. Signorile-Signorile, P. Torrielli, S. Uccirati, JHEP 1812, 107 (2018)

28. L. Magnea, E. Maina, G. Pelliccioli, C. Signorile-Signorile, P. Torrielli, S. Uccirati, JHEP 1812, 062 (2018)

29. F. Herzog, JHEP 1808, 006 (2018)

30. F. Caola, K. Melnikov, R. Röntsch, Eur. Phys. J. C 77(4), 248 (2017)

31. F. Caola, G. Luisoni, K. Melnikov, R. Röntsch, Phys. Rev. D 97(7), 074022 (2018)

32. F. Caola, M. Delto, H. Frellesvig, K. Melnikov, Eur. Phys. J. C 78(8), 687 (2018)

33. M. Delto, K. Melnikov. arXiv:1901.05213 [hep-ph]

34. R. Hamberg, W.L. van Neerven, T. Matsuura, Nucl. Phys. B 359, 343 (1991). Erratum: [Nucl. Phys. B 644, 403 (2002)]

35. C. Anastasiou, K. Melnikov, Nucl. Phys. B 646, 220 (2002)

36. S. Frixione, Z. Kunszt, A. Signer, Nucl. Phys. B 467, 399 (1996)

37. S. Frixione, Nucl. Phys. B 507, 295 (1997)

38. R.K. Ellis, W.J. Stirling, B.R. Webber, Camb. Monogr. Part. Phys. Nucl. Phys. Cosmol. 8, 1 (1996)

39. S. Catani, M. Grazzini, Nucl. Phys. B 570, 287 (2000)

40. R.D. Ball et al. [NNPDF Collaboration], JHEP 1504, 040 (2015)

41. G.P. Salam, J. Rojo, Comput. Phys. Commun. 180, 120 (2009)

42. M. Grazzini, S. Kallweit, M. Wiesemann, Eur. Phys. J. C 78(7), 537 (2018)

43. S. Catani, Phys. Lett. B 427, 161 (1998)

44. T. Gehrmann, T. Huber, D. Maitre, Phys. Lett. B 622, 295 (2005)

45. J. Grigo, K. Melnikov, M. Steinhauser, Nucl. Phys. B 888, 17 (2014) 